\title{
Design-Based Ratio Estimators and Central Limit Theorems for Clustered, Blocked RCTs
}

\section{February 2021}

\author{
Peter Z. Schochet, Ph.D. (Corresponding Author) \\ Senior Fellow, Mathematica \\ P.O. Box 2393 \\ Princeton, NJ 08543-2393 \\ Phone: (609) 936-2783 \\ pschochet@mathematica-mpr.com \\ Nicole E. Pashley \\ Rutgers University \\ 110 Frelinghuysen Road \\ Piscataway, NJ 08854 \\ nicole.pashley@ rutgers.edu \\ Luke W. Miratrix, Ph.D. \\ Graduate School of Education \\ Harvard University \\ 14 Appian Way \\ Cambridge, MA 02138 \\ luke_miratrix@gse.harvard.edu \\ Tim Kautz, Ph.D. \\ Senior Researcher, Mathematica \\ P.O. Box 2393 \\ Princeton, NJ 08543-2393 \\ Phone: (609) 297-4544 \\ tkautz@mathematica-mpr.com
}




\begin{abstract}
This article develops design-based ratio estimators for clustered, blocked randomized controlled trials (RCTs), with an application to a federally funded, school-based RCT testing the effects of behavioral health interventions. We consider finite population weighted least squares estimators for average treatment effects (ATEs), allowing for general weighting schemes and covariates. We consider models with block-by-treatment status interactions as well as restricted models with block indicators only. We prove new finite population central limit theorems for each block specification. We also discuss simple variance estimators that share features with commonly used cluster-robust standard error estimators. Simulations show that the design-based ATE estimator yields nominal rejection rates with standard errors near true ones, even with few clusters.
\end{abstract}

Keywords: Randomized controlled trials; clustered designs; blocked designs; design-based estimators; finite population central limit theorems

\title{
Acknowledgements
}

The authors thank Charles Tilley, Joel Middleton, and session participants at the Society for Research on Education Effectiveness conference for useful feedback on the manuscript. Nicole Pashley is supported by the National Science Foundation Graduate Research Fellowship under Grant No. DGE1745303. Any opinion, findings, and conclusions or recommendations expressed in this material are those of the authors and do not necessarily reflect the views of the National Science Foundation. 


\section{Introduction}

There is a growing literature on design-based methods for analyzing randomized controlled trials (RCTs) (e.g., Yang and Tsiatis 2001; Freedman (2008); Schochet 2010, 2016; Lin 2013; Miratrix et al. 2013; Imbens and Rubin 2015; Middleton and Aronow 2015; Li and Ding 2017). These nonparametric methods are built on the potential outcomes framework, introduced by Neyman (1923) and later developed in seminal works by Rubin (1974, 1977) and Holland (1986). They leverage a fundamental component of experimental designs - the known treatment assignment mechanism—to achieve results that rely on minimal assumptions.

The design-based literature has largely focused on non-clustered designs in which individuals are randomly assigned to research conditions. A much smaller literature has considered designbased methods for clustered RCTs where groups (such as schools, hospitals, or communities) rather than individuals are randomized. Clustered designs are common in evaluations that test interventions targeted to a group and are sometimes preferred to non-clustered designs as they can help minimize bias due to the potential spillover of intervention effects from treatment to control subjects. Clustered designs are becoming increasingly prevalent in social policy research (Schochet 2008) and have grown exponentially in medical trials (Bland 2004).

For example, the evaluation of the Social and Character Development (SACD) Research Program was a major federal initiative, co-funded by the Institute of Education Sciences at the U.S. Department of Education and the Centers for Disease Control and Prevention, to test interventions promoting positive social and character development among elementary school children, with the goal of ultimately improving their academic performance (SACD Consortium 2010). The study was conducted in seven large school districts (blocks), where half the schools (clusters) within each district were randomly assigned to a treatment group and half to a control 
group, yielding a final sample of 84 schools (42 treatment and 42 control). Intervention features included materials and lessons on social skills, behavior management, social and emotional learning, self-control, anger management, and violence prevention.

Several key aspects of the SACD study motivate the theory underlying this article. First, neither the sample of seven SACD school districts nor the 10 to 14 study schools per district were randomly sampled from broader populations. Rather, the participating districts and schools were volunteers, yielding a convenience sample, as is often the case in RCTs across disciplines. This suggests a finite population framework for estimating average treatment effects (ATEs), where the sample and their potential outcomes are considered fixed, with treatment assignments being the only source of randomness, and where study results are assumed to pertain to the study sample only (Neyman 1923). This framework differs from typical model-based, super-population approaches where potential outcomes are assumed to be randomly sampled from a broader (often infinite) population — even if vaguely defined — and where study results are assumed to generalize to this population.

A second aspect of the SACD study that motivates our theory is the need for flexible weighting schemes to accommodate decisions on how clusters and blocks are to be weighted to estimate pooled effects and to help adjust for nonresponse. Third, the theory should address common approaches for including and incorporating block (fixed) effects in the models. Finally, the estimation strategy should allow for the inclusion of model baseline covariates to improve precision; this is especially important for clustered designs where power is often a concern due to design effects from clustering and the typical high cost of adding clusters to the study.

We achieve these objectives in this work by developing covariate-adjusted design-based methods for obtaining point estimates and associated inference for clustered RCTs. Our results 
rely on new finite population central limit theorems (CLTs) for design-based ATE ratio estimators that apply to the general case where randomization of clusters is conducted within blocks (strata). We consider ratio estimators for clustered RCTs obtained using weighted least squares (WLS) methods, which have intuitive appeal because they parallel differences-in-means and regression-adjusted ATE estimators for non-clustered designs. We allow for general weighting schemes and covariates. We also consider models with block-by-treatment status interactions as well as models with block fixed effects only.

We provide consistent variance estimators and compare them, both analytically and through simulations, to widely used ordinary least squares estimators with cluster-robust standard errors (CRSE) (Liang and Zeger 1986; Cameron and Miller 2011). Our simulations suggest that the design-based ratio estimators yield Type 1 error rates near nominal levels, even with relatively few clusters. We also conduct an empirical analysis using data from the SACD study to compare different specifications of our estimators to each other and to the standard CRSE estimator.

The rest of this article is structured as follows. Section 2 discusses the literature this work is built on and Section 3 provides our theoretical framework. Sections 4 and 5 present our finite population CLTs and variance estimators. Section 6 presents simulation results and Section 7 presents empirical results using our motivating SACD example. Section 8 concludes.

\section{Related Work}

Our finite population CLTs build on Li and Ding (2017), who consider CLTs for unbiased estimators for clustered RCTs using the Horvitz-Thompson estimator developed by Middleton and Aronow (2015) and allow for cluster-level covariates, but do not consider ratio estimators or blocked designs with general weighting schemes. Our theory also builds on results in Scott and Wu (1981) who consider CLTs for ratio estimators for finite population totals, but not for 
clustered designs or RCTs. We extend the design-based results in Imai et al. (2009) who examine clustered RCTs with pairwise matching but not general blocked designs, models with covariates, or CLTs. We also extend the design-based results in Schochet (2013) who examines clustered designs without blocking, and Pashley and Miratrix (2017) and Liu and Yang (2020) who consider blocked designs without clustering. In particular, Liu and Yang (2020) also derive finite population CLTs for blocked designs with covariates for RCTs, but without clustering.

Other literature in this area has a different focus. Abadie et al. (2017) discuss reasons for adjusting for clustering and investigate differences between the true asymptotic finite population variance and the CRSE variance estimator, but do not consider impact estimation. Hansen and Bowers (2009) propose model-assisted estimators combined with randomization inference for regression models in a specific context without deriving design-based estimators. Samii and Aronow (2012) compare design-based and robust estimators for non-clustered designs, but not for clustered designs or models with covariates. While there is a large statistical literature on related design-based methods for analyzing survey data with complex sample designs (e.g., Fuller 1975, 2009; Cochran 1977; Bickel and Freedman, 1984; Rao and Shao 1999; Wolter 2007; Lohr 2009), these works do not focus on RCT settings.

\section{Framework and Definitions}

We assume that a clustered RCT of $m$ total clusters is conducted across $h$ blocks, with block $b$ having $m_{b}$ clusters $(b=1, \ldots, h)$. Randomization of clusters is conducted separately by block, with $m_{b}^{1}=m_{b} p_{b}$ assigned to the treatment group and $m_{b}^{0}=m_{b}\left(1-p_{b}\right)$ assigned to the control group $\left(0<p_{b}<1\right)$. We assume a sample of $n_{j b}$ individuals in cluster $j$ in block $b$, with $n_{b}$ individuals in the block and $n$ individuals in total. For each cluster, either all individuals are

treated or not. We index individuals by $i j b$ for individual $i$ in cluster $j$ in block $b$. Let $Y_{i j b}(1)$ be 
a person's outcome if assigned to a treated cluster and $Y_{i j b}(0)$ be the outcome in a control cluster. These potential outcomes can be continuous, binary, or discrete. We assume a finite population model, where potential outcomes are assumed to be fixed for the study. Let $T_{j b}$ equal 1 if cluster $j b$ is randomly assigned to the treatment condition and 0 otherwise. Let $S_{i j b, s}$ and $S_{j b, s}$ denote indicator variables of block membership for individuals and clusters (that is, $S_{i j b, s}=$ 1 or $S_{j b, s}=1$ if the specified person or cluster belongs to block $s$ ).

We also allow for weights, with individual weights of $w_{i j b}>0$, cluster weights of $w_{j b}=$ $\sum_{i=1}^{n_{j b}} w_{i j b}$, and block weights of $w_{b}=\sum_{j=1}^{m_{b}} w_{j b}$. Depending on the research questions of interest, the weights can be set, for example, so that intervention effects pertain to the average individual in the block $\left(w_{i j b}=1\right.$ and $\left.w_{j b}=n_{j b}\right)$ or the average cluster in the block $\left(w_{i j b}=1 / n_{j b}\right.$ and $w_{j b}=1$ ). They can also be further modified to handle various forms of data nonresponse (we do not consider estimation error in the nonresponse weights in our variance formulas).

We assume two conditions that generalize those in Imbens and Rubin (2015) for the nonclustered RCT design to our context. The first is the stable unit treatment value assumption (SUTVA) (Rubin 1986):

(C1): SUTVA: Let $Y_{i j b}\left(\mathbf{T}_{c l u s}\right)$ denote the potential outcome for an individual given the random vector of all cluster treatment assignments, $\mathbf{T}_{c l u s}$. Then, if $T_{j b}=T_{j b}^{\prime}$ for cluster $j$, we have that $Y_{i j b}\left(\mathbf{T}_{\text {clus }}\right)=Y_{i j b}\left(\mathbf{T}_{\text {clus }}^{\prime}\right)$.

SUTVA allows us to express $Y_{i j b}\left(\mathbf{T}_{c l u s}\right)$ as $Y_{i j b}\left(T_{j b}\right)$, so that the vector of individual potential outcomes in cluster $j b$ depends only on the cluster's treatment assignment and not on the treatment assignments of other clusters in the sample. SUTVA could be more plausible for clustered designs than non-clustered designs because there are likely to be fewer meaningful 
interactions between sample members across clusters than within clusters. SUTVA also assumes a particular treatment unit cannot receive different forms of the treatment.

Under SUTVA, the block $b$ ATE parameter of interest for the finite population model is

$$
\beta_{1, b}=\frac{\sum_{j=1}^{m_{b}} w_{j b}\left(\bar{Y}_{j b}(1)-\bar{Y}_{j b}(0)\right)}{\sum_{j=1}^{m_{b}} w_{j b}}=\overline{\bar{Y}}_{b}(1)-\overline{\bar{Y}}_{b}(0),
$$

where, for $t \in\{1,0\}, \bar{Y}_{j b}(t)=\frac{1}{w_{j b}} \sum_{i=1}^{n_{j b}} w_{i j b} Y_{i j b}(t)$ is the weighted mean potential outcome in the treatment or control condition. The ATE parameter across all blocks is then a weighted average of the block-specific ATE parameters:

$$
\beta_{1}=\frac{\sum_{b=1}^{h} w_{b} \beta_{1, b}}{\sum_{b=1}^{h} w_{b}}
$$

Our second condition is the randomization itself:

(C2): Complete randomization of clusters within blocks: Let $\mathbf{T}_{c l u s, b}$ be the random vector of cluster treatment assignments in block $b$. Let $\left(m_{1}^{1}, \ldots, m_{h}^{1}\right)$ be a pre-specified vector denoting the number of clusters to assign to treatment within each block. Then, for any vector, $\mathbf{t}_{c l u s, b}=$ $\left(t_{1 b}, \ldots, t_{m_{b} b}\right)$ of randomization realizations such that $\sum_{j=1}^{m_{b}} t_{j b}=m_{b}^{1}$, we have that $\operatorname{prob}\left(\mathbf{T}_{c l u s, b}=\mathbf{t}_{c l u s, b}\right)=\left(\begin{array}{c}m_{b} \\ m_{b}^{1}\end{array}\right)^{-1}$. This holds for all $b \in\{1, \ldots, h\}$, and we further assume that the cluster assignments, $\left(\mathbf{T}_{c l u s, 1}, \ldots, \mathbf{T}_{c l u s, h}\right)$, are mutually independent.

\section{ATE Estimators for the Finite Population Model}

Under the potential outcomes framework and SUTVA, the data generating process for the observed outcome measure, $y_{i j b}$, is a consequence of the assignment mechanism:

$$
y_{i j b}=T_{j b} Y_{i j b}(1)+\left(1-T_{j b}\right) Y_{i j b}(0)
$$


This relation states that we can observe $y_{i j b}=Y_{i j b}(1)$ for those in the treatment group and $y_{i j b}=Y_{i j b}(0)$ for those in the control group, but not both.

Rearranging (3) generates the following nominal regression model for any given block:

$$
y_{i j b}=\beta_{0, b}+\beta_{1, b}\left(T_{j b}-p_{b}^{*}\right)+u_{i j b}
$$

where $\beta_{1, b}=\overline{\bar{Y}}_{b}(1)-\overline{\bar{Y}}_{b}(0)$ is the block-specific ATE parameter, $p_{b}^{*}=\frac{1}{w_{b}} \sum_{j=1}^{m_{b}} T_{j b} w_{j b}$ is the weighted treatment group assignment probability, $\beta_{0, b}=p_{b}^{*} \overline{\bar{Y}}_{b}(1)+\left(1-p_{b}^{*}\right) \overline{\bar{Y}}_{b}(0)$ is the mean potential outcome in the block, and the "error" term, $u_{i j b}$, can be expressed as

$$
u_{i j b}=T_{j b}\left(Y_{i j b}(1)-\overline{\bar{Y}}_{b}(1)\right)+\left(1-T_{j b}\right)\left(Y_{i j b}(0)-\overline{\bar{Y}}_{b}(0)\right)
$$

We center the treatment indicator in (4) to facilitate the theory without changing the estimator.

In contrast to usual formulations of the regression model, our residual, $u_{i j b}$, is random solely because of $T_{j b}$ (that is, due to random assignment) (see also Freedman 2008, Lin 2013 and Middleton 2018). This framework allows treatment effects to differ across individuals and clusters and is nonparametric because it makes no assumptions about the distribution of potential outcomes. Note that our model does not satisfy key assumptions of the usual regression model for correlated data: over the randomization distribution, $E\left(u_{i j b}\right)$ is not zero, $u_{i j b}$ is heteroscedastic, $\operatorname{Cov}\left(u_{i j b}, u_{i^{\prime} j b}\right)$ is not constant for individuals in the same cluster, $\operatorname{Cov}\left(u_{i j b}, u_{i^{\prime} j^{\prime} b}\right)$ is nonzero for individuals in different clusters, and $u_{i j b}$ is correlated with the regressor $\left(T_{j b}-p_{b}^{*}\right)$ (see Schochet 2016). Under this framework, correlations arise because individuals in the same cluster share the same treatment assignment, and because $T_{j b}$ and $T_{j^{\prime} b}$ are correlated due to the complete randomization of clusters within the finite population. This differs from the typical model-based framework where correlations arise from shared cluster-specific random effects (e.g., due to common environmental factors). 
The model in (4) can also be expressed using block indicator variables as follows:

$$
y_{i j b}=\sum_{s=1}^{h} \beta_{1, s} S_{i j b, s} \tilde{T}_{j s}+\sum_{s=1}^{h} \beta_{0, s} S_{i j b, s}+u_{i j b}
$$

where $\tilde{T}_{j b}=\left(T_{j b}-p_{b}^{*}\right)$ is the block centered treatment status indicator. Due to blocked random assignment, the errors are independent across blocks. We include terms for all $h$ blocks in the model and exclude a grand intercept term.

For estimation, we use the following working (hypothesized) model that provides covariateadjusted ATE estimates by including in (5) a $1 x v$ vector of fixed, block-mean centered baseline covariates, $\tilde{\mathbf{x}}_{i j b}$, with associated parameter vector $\boldsymbol{\gamma}$ :

$$
y_{i j b}=\sum_{s=1}^{h} \beta_{1, k} S_{i j b, s} \tilde{T}_{j s}+\sum_{s=1}^{h} \beta_{0, s} S_{i j b, s}+\tilde{\mathbf{x}}_{i j b} \boldsymbol{\gamma}+e_{i j b}
$$

where $\tilde{\mathbf{x}}_{i j b}=\left(\mathbf{x}_{i j b}-\overline{\mathbf{x}}_{b}\right), \overline{\mathbf{x}}_{b}=\frac{1}{w_{b}} \sum_{j=1}^{m_{b}} \sum_{i=1}^{n_{j b}} w_{i j b} \mathbf{x}_{i j b}$, and $e_{i j b}$ is the error term. These covariates, unaffected by the treatment, can be at the individual or cluster level. We assume sufficient degrees of freedom for variance estimation (see Section 4.2). While the covariates do not enter the true RCT model in (4) and the ATE estimands do not change, the covariates will increase precision to the extent they are correlated with the potential outcomes. We do not need to assume that the true conditional distribution of $y_{i j b}$ given $\mathbf{x}_{i j b}$ is linear in $\mathbf{x}_{i j b}$.

We do not consider working models that interact $\tilde{\mathbf{x}}_{i j b}$ and $\widetilde{T}_{j b}$ due to associated degrees of freedom losses that can seriously reduce the power of clustered RCTs that, in practice, often contain relatively few clusters for cost reasons. Similarly, we pool $\boldsymbol{\gamma}$ across blocks. As discussed in Section 4.1, $\gamma$ is well defined: it is the finite population regression coefficient that would be obtained if we could run the weighted regression on the full schedule of potential outcomes. 
Using individual-level data, we can fit our working model using weighted least squares (WLS) with weights $w_{i j b}$. This yields the following closed-form WLS estimator for $\beta_{1, b}$ (see Section A.3 in the supplementary materials for the derivation):

$$
\begin{gathered}
\hat{\beta}_{1, b}=\frac{1}{w_{b}^{1}} \sum_{j: T}^{m_{b}=1} w_{j b} \bar{y}_{j b}-\frac{1}{w_{b}^{0}} \sum_{j: T_{j b}=0}^{m_{b}} w_{j b} \bar{y}_{j b}-\left(\frac{1}{w_{b}^{1}} \sum_{j: T_{j b}=1}^{m_{b}} w_{j b} \overline{\mathbf{x}}_{j b}-\frac{1}{w_{b}^{0}} \sum_{j: T_{j b}=0}^{m_{b}} w_{j b} \overline{\mathbf{x}}_{j b}\right) \hat{\boldsymbol{\gamma}} \\
=\overline{\bar{y}}_{b}(1)-\overline{\bar{y}}_{b}(0)-\left(\overline{\mathbf{x}}_{b}^{1}-\overline{\mathbf{x}}_{b}^{0}\right) \hat{\boldsymbol{\gamma}}
\end{gathered}
$$

where, for $t \in\{1,0\}, \overline{\bar{y}}_{b}(t)$ is the weighted average of the observed outcome across subjects in the treatment or control group, $w_{b}^{t}=\sum_{j: T}^{m_{b}}{ }_{j b}=w_{j b}$ is the sum of the weights, and $\widehat{\gamma}$ is the WLS

estimator for $\boldsymbol{\gamma} \cdot \hat{\beta}_{1, b}$ is the WLS estimator that is obtained using standard statistical packages.

\subsection{Theoretical Results}

To consider the asymptotic properties of $\hat{\beta}_{1, b}$, we consider a hypothetical increasing sequence of finite populations where $m_{b} \rightarrow \infty$ in each block, so that $m=\sum_{b=1}^{h} m_{b} \rightarrow \infty$. The number of blocks, $h$, however, remains fixed. In principle, parameters should be subscripted by $m$, but we omit this notation for simplicity. We further assume that the proportion of all clusters in a block converges to a constant, that is, $m_{b} / m \rightarrow q_{b}$ as $m \rightarrow \infty$. We finally assume that $p_{b}=m_{b}^{1} / m_{b}$ is (approximately) constant as $m \rightarrow \infty$, so that the number of treated and control clusters in each block increases with $m$ (that is, $m_{b}^{1} \rightarrow \infty$ and $m_{b}^{0} \rightarrow \infty$ ).

Given this framework, we present a CLT for the WLS estimator that provides design-based standard errors and associated inference. Before presenting our theorem, we first need to define several quantities pertaining to finite population variances and covariances. First, for $t \in\{1,0\}$, we define $D_{b}(t)=\frac{w_{j b}}{\bar{w}_{b}}\left(\bar{Y}_{j b}(t)-\overline{\bar{Y}}_{b}(t)-\left(\overline{\mathbf{x}}_{j b}-\overline{\overline{\mathbf{x}}}_{b}\right) \boldsymbol{\gamma}\right)$ as the residualized potential outcomes 
at the cluster level in the treatment and control conditions, where $\bar{w}_{b}=\frac{1}{m_{b}} \sum_{j=1}^{m_{b}} w_{j b}$. Second, we define $S_{D_{b}}^{2}(t)$ as the variance of these residuals,

$$
S_{D_{b}}^{2}(t)=\frac{1}{m_{b}-1} \sum_{j=1}^{m_{b}} \frac{w_{j b}^{2}}{\bar{w}_{b}^{2}}\left(\bar{Y}_{j b}(t)-\overline{\bar{Y}}_{b}(t)-\left(\overline{\mathbf{x}}_{j b}-\overline{\overline{\mathbf{x}}}_{b}\right) \boldsymbol{\gamma}\right)^{2}
$$

and $S_{D_{b}}^{2}(1,0)$ as the associated treatment-control covariance,

$$
S_{D_{b}}^{2}(1,0)=\frac{1}{m_{b}-1} \sum_{j=1}^{m_{b}} \frac{w_{j b}^{2}}{\bar{w}_{b}^{2}}\left(\bar{Y}_{j b}(1)-\overline{\bar{Y}}_{b}(1)-\left(\overline{\mathbf{x}}_{j b}-\overline{\overline{\mathbf{x}}}_{b}\right) \boldsymbol{\gamma}\right)\left(\bar{Y}_{j b}(0)-\overline{\bar{Y}}_{b}(0)-\left(\overline{\mathbf{x}}_{j b}-\overline{\overline{\mathbf{x}}}_{b}\right) \boldsymbol{\gamma}\right) .
$$

Third, we define $\operatorname{Var}\left(\hat{D}_{b}\right)$ as the variance of the mean difference in residuals between the observed (randomized) treatment and control group samples,

$$
\operatorname{Var}\left(\hat{D}_{b}\right)=\frac{S_{D_{b}}^{2}(1)}{m_{b}^{1}}+\frac{S_{D_{b}}^{2}(0)}{m_{b}^{0}}-\frac{S^{2}\left(D_{b}\right)}{m_{b}}
$$

where $S^{2}\left(D_{b}\right)$ is the variance (heterogeneity) of the ATEs across clusters in block $b$,

$$
S^{2}\left(D_{b}\right)=\frac{1}{m_{b}-1} \sum_{j=1}^{m_{b}} \frac{w_{j b}^{2}}{\bar{w}_{b}^{2}}\left(\bar{Y}_{j b}(1)-\bar{Y}_{j b}(0)-\left(\overline{\bar{Y}}_{b}(1)-\overline{\bar{Y}}_{b}(0)\right)\right)^{2} .
$$

Fourth, we define the variance of the weights as $S^{2}\left(w_{b}\right)=\frac{1}{m_{b}-1} \sum_{j=1}^{m_{b}}\left(w_{j b}-\bar{w}_{b}\right)^{2}$. Fifth, we need the weighted variances, $S_{x_{b}, k}^{2}$, of each covariate $k$,

$$
S_{x_{b}, k}^{2}=\frac{1}{m_{b}-1} \sum_{j=1}^{m_{b}} \frac{w_{j b}^{2}}{\bar{w}_{b}^{2}}\left(\left[\overline{\mathbf{x}}_{j b}-\overline{\overline{\mathbf{x}}}_{b}\right]_{k}\right)^{2},
$$

and the weighted variance-covariance matrix of the covariates with themselves, $\mathbf{S}_{\mathbf{x}, b}^{2}$, which is analogous to the classic $\mathbf{X}^{\prime} \mathbf{W X}$ matrix in WLS,

$$
\mathbf{S}_{\mathbf{x}, b}^{2}=\frac{1}{m_{b}} \sum_{j=1}^{m_{b}} \sum_{i=1}^{n_{j b}} w_{i j b}\left(\mathbf{x}_{i j b}-\overline{\overline{\mathbf{x}}}_{b}\right)^{\prime}\left(\mathbf{x}_{i j b}-\overline{\overline{\mathbf{x}}}_{b}\right) .
$$


Finally, we need two matrices that are analogous to the classic $\mathbf{X}^{\prime} \mathbf{W Y}$ matrix in WLS,

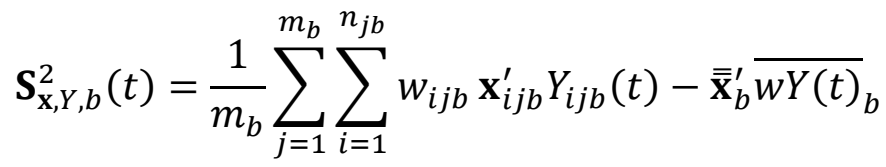

and

$$
\mathbf{S}_{\mathbf{x} Y, b}^{2}(t)=\frac{1}{m_{b}} \sum_{j=1}^{m_{b}} \sum_{i=1}^{n_{j b}}\left(w_{i j b} \mathbf{x}_{i j b}^{\prime} Y_{i j b}(t)-\overline{w x Y(t)}\right)_{b}^{2}
$$

where $\overline{w Y(t)_{b}}=\frac{1}{m_{b}} \sum_{j=1}^{m_{b}} \sum_{i=1}^{n_{j b}} w_{i j b} Y_{i j b}(t)$ and $\overline{w x Y(t)}{ }_{b}=\frac{1}{m_{b}} \sum_{j=1}^{m_{b}} \sum_{i=1}^{n_{j b}} w_{i j b} \mathbf{x}_{i j b}^{\prime} Y_{i j b}(t)$.

We now present our CLT theorem, proved in Section A.3 in the supplementary materials, which adapts finite population CLT results in Li and Ding (2017) and Scott and Wu (1981) to our setting.

Theorem 1. Assume (C1), (C2), and the following conditions for $t \in\{1,0\}$ and $b \in$ $\{1,, \ldots, h\}:$

(C3) Letting $g_{b}(t)=\max _{1 \leq j \leq m_{b}}\left(\frac{w_{j b}}{\bar{w}_{b}}\left(\bar{Y}_{j b}(t)-\overline{\bar{Y}}_{b}(t)-\left(\overline{\mathbf{x}}_{j b}-\overline{\overline{\mathbf{x}}}_{b}\right) \boldsymbol{\gamma}\right)\right)^{2}$, as $m \rightarrow \infty$,

$$
\frac{1}{\left(m_{b}^{t}\right)^{2}} \frac{g_{b}(t)}{\operatorname{Var}\left(\hat{D}_{b}\right)} \rightarrow 0
$$

(C4) $f_{b}^{t}=m_{b}^{t} / m_{b}$ has a limiting value in $(0,1)$ and $S_{D_{b}}^{2}(t)$, and $S_{D_{b}}^{2}(1,0)$ also have finite limiting values.

(C5) As $m \rightarrow \infty$,

$$
\left(1-f_{b}^{t}\right) \frac{S^{2}\left(w_{b}\right)}{m_{b}^{t} \bar{w}_{b}^{2}} \rightarrow 0
$$

(C6) Letting $h_{b, k}(t)=\max _{1 \leq j \leq m_{b}}\left\{\frac{w_{j b}}{\bar{w}_{b}}\left(\left[\bar{x}_{j b}-\overline{\bar{x}}_{b}\right]_{k}\right)\right\}^{2}$ for all $k$, as $m \rightarrow \infty$,

$$
\frac{1}{\min \left(m_{b}^{1}, m_{b}^{0}\right)} \frac{h_{b, k}(t)}{S_{x_{b}, k}^{2}} \rightarrow 0
$$

(C7) $S_{x_{b}, k}^{2}, \mathbf{S}_{\mathbf{x}, b}^{2}, \mathbf{S}_{\mathbf{x}, Y, b}^{2}(t)$, and $\mathbf{S}_{\mathbf{x} Y, b}^{2}(t)$ have finite (positive definite) limiting values. 
Then, as $m \rightarrow \infty, \hat{\beta}_{1, b}$ is a consistent estimator for $\beta_{1, b}$ and

$$
\frac{\hat{\beta}_{1, b}-\left(\overline{\bar{Y}}_{b}(1)-\overline{\bar{Y}}_{b}(0)\right)}{\sqrt{\operatorname{Var}\left(\hat{D}_{b}\right)}} \stackrel{d}{\rightarrow} N(0,1)
$$

where $\operatorname{Var}\left(\hat{D}_{b}\right)$ is defined as in (8).

Remark 1. Condition (C3) is a Lindeberg-type condition (controlling the tails) that allows us to invoke the CLT in Theorem 4 of Li and Ding (2017) that underlies our finite population CLT. (C4) ensures that the treatment and control group samples in each block both grow sufficiently fast, and also ensures limiting values of asymptotic variances and covariances of the residualized potential outcomes. (C5) provides a weak law of large numbers for the weights so that $\bar{w}_{b}^{t} / \bar{w}_{b} \stackrel{p}{\rightarrow}$ 1 , where $\bar{w}_{b}^{t}=\frac{1}{m_{b}^{t}} \sum_{j: T_{j}=t}^{m_{b}} w_{j b}$. (C7) specifies limiting values of the covariate variances and outcome-covariate covariances, which in turn, provide regularity conditions on $\hat{\boldsymbol{\gamma}}$.

These conditions imply that within a block, we cannot have one cluster (or a few clusters) asymptotically dominating all other clusters in terms of their weights, so that the weighted covariance matrices for the outcomes and covariates are well defined. In general, this is unlikely to be restrictive: due to normalizing by the mean block weight, even if weighted cluster sizes steadily grow, our results hold as long as the relative block sizes do not get too disparate.

Remark 2 . The above theorem is proved as a two-step process. We first assume $\boldsymbol{\gamma}=$ $\left(\sum_{b=1}^{h} q_{b} \mathbf{S}_{\mathbf{x}, b}^{2}\right)^{-1}\left[\sum_{b=1}^{h} p_{b} q_{b} \mathbf{S}_{\mathbf{x}, Y, b}^{2}(1)+\sum_{b=1}^{h}\left(1-p_{b}\right) q_{b} \mathbf{S}_{\mathbf{x}, Y, b}^{2}(0)\right]$ is known, where $q_{b}=m_{b} / m$ and $p_{b}=m_{b}^{1} / m_{b}$, and obtain a CLT with this known parameter. This parameter is the (unobserved) WLS coefficient vector that would be obtained using the full set of potential outcomes. We then show that $\hat{\boldsymbol{\gamma}}$ converges to the same asymptotic value as $\boldsymbol{\gamma}$ and use (C6) to 
ensure that $\left(\overline{\overline{\mathbf{x}}}_{b}^{1}-\overline{\mathbf{x}}_{b}^{0}\right)$ is asymptotically normal with zero mean, so that the ATE estimator still converges to a standard normal. Note that we focus on the asymptotic regime where the number of clusters increases. Liu and Yang (2020) instead allow the number of blocks to increase, focusing on settings with a small number of clusters per block and a large number of blocks.

Remark 3. The first two terms in (8) pertain to separate variances for the treatment and control groups because we allow for heterogeneous treatment effects. These variances are based on model residuals averaged to the cluster level and are similar in form to the variance formulas in Li and Ding (2017) for non-blocked designs. The third term pertains to the covariance of cluster-level average potential outcomes in the treatment and control conditions, $S_{D_{b}}^{2}(1,0)$, that we express in terms of the heterogeneity of treatment effects across clusters, $S^{2}\left(D_{b}\right)$. We hereafter label this term the "finite population heterogeneity" term. It cannot be identified from the data but can be bounded (as discussed in Section 4.2).

Remark 4. Under (C1)-(C5), Theorem 1 also applies to models without covariates by setting $\boldsymbol{\gamma}=\mathbf{0}$, yielding a simple differences-in-means ATE ratio estimator, $\hat{\beta}_{1, b}=\overline{\bar{y}}_{b}(1)-\overline{\bar{y}}_{b}(0)$. We discuss the finite sample bias of this estimator in Section A.2.2 in the supplementary materials.

Corollary 1. Under the conditions of Theorem 1 with model covariates, the asymptotic variance in (8) is minimized when $\boldsymbol{\gamma}=\boldsymbol{\gamma}_{\mathbf{B}}$, where $\boldsymbol{\gamma}_{\mathbf{B}}$ is the between-cluster regression parameter using data aggregated to the cluster level. The parameter, $\boldsymbol{\gamma}_{\mathbf{B}}$, is defined analogously to $\boldsymbol{\gamma}$ in Remark 2 by replacing $\mathbf{S}_{\mathbf{x}, b}^{2}$ with $\mathbf{S}_{\mathbf{x}, b, \mathbf{B}}^{2}=\frac{1}{m_{b}} \sum_{j=1}^{m_{b}} w_{j b}\left(\overline{\mathbf{x}}_{j b}-\overline{\mathbf{x}}_{b}\right)^{\prime}\left(\overline{\mathbf{x}}_{j b}-\overline{\overline{\mathbf{x}}}_{b}\right)$, and using parallel cluster-level versions of $\mathbf{S}_{\mathbf{x}, Y, b}^{2}(1)$ and $\mathbf{S}_{\mathbf{x}, Y, b}^{2}(0)$.

This result follows because $S_{D_{b}}^{2}(1)$ and $S_{D_{b}}^{2}(0)$ in (8) are based on cluster-level residuals. Section A.5 of the supplementary materials derives the asymptotic efficiency loss using the individual data when the covariates vary both within and between clusters. Intuitively, the $\boldsymbol{\gamma}$ 
parameter is a weighted average of between- and within-cluster population regression

parameters. But the within-cluster covariates, $\left(\mathbf{x}_{i j}-\overline{\mathbf{x}}_{j}\right)$, have no effect on $S_{D_{b}}^{2}(t)$, and hence, on precision, so they bias $\boldsymbol{\gamma}$. However, as discussed in Section 4.2, these efficiency losses using the individual data can be offset by other precision factors in finite samples.

Corollary 2. Under the conditions of Theorem 1 and assuming $\bar{w}_{b}=\frac{w_{b}}{m_{b}}$ has a finite limit for all $b$, the pooled ATE estimator across blocks, $\hat{\beta}_{1}=\frac{1}{h \bar{w}} \sum_{b=1}^{h} w_{b} \hat{\beta}_{1, b}$, is consistent for $\beta_{1}$ in (2) and $\frac{1}{\sqrt{\operatorname{Var}(\widehat{D})}}\left(\hat{\beta}_{1}-\beta_{1}\right) \stackrel{d}{\rightarrow} N(0,1)$, where $\operatorname{Var}(\widehat{D})=\frac{1}{(h \bar{w})^{2}} \sum_{b=1}^{h} w_{b}^{2} \operatorname{Var}\left(\widehat{D}_{b}\right)$ and $\bar{w}=\frac{1}{h} \sum_{b=1}^{h} w_{b}$.

This result follows because the $\hat{\beta}_{1, b}$ estimators are asymptotically independent.

\subsection{Variance Estimation}

We can estimate the block-specific variance in (8) with a consistent (upper bound) plug-in variance estimator based on the regression residuals averaged to the cluster level as follows:

where

$$
\operatorname{Vâr}\left(\hat{D}_{b}\right)=\frac{s_{D_{b}}^{2}(1)}{m_{b}^{1}}+\frac{s_{D_{b}}^{2}(0)}{m_{b}^{0}},
$$

$$
\begin{aligned}
& s_{D_{b}}^{2}(1)=\frac{1}{\left(m_{b}^{1}-v^{*} p_{b}^{*} q_{b}^{*}-1\right)} \sum_{j: T_{j b}=1}^{m_{b}^{1}} \frac{w_{j b}^{2}}{\left(\bar{w}_{b}^{1}\right)^{2}}\left(\bar{y}_{j b}-\hat{\beta}_{0, b}-\left(1-p_{b}^{*}\right) \hat{\beta}_{1, b}-\tilde{\overline{\mathbf{x}}}_{j b} \hat{\boldsymbol{\gamma}}\right)^{2}, \\
& s_{D_{b}}^{2}(0)=\frac{1}{\left(m_{b}^{0}-v^{*}\left(1-p_{b}^{*}\right) q_{b}^{*}-1\right)} \sum_{j: T_{j b}=0}^{m_{b}^{0}} \frac{w_{j b}^{2}}{\left(\bar{w}_{b}^{0}\right)^{2}}\left(\bar{y}_{j b}-\hat{\beta}_{0, b}+p_{b}^{*} \hat{\beta}_{1, b}-\tilde{\overline{\mathbf{x}}}_{j b} \hat{\boldsymbol{\gamma}}\right)^{2},
\end{aligned}
$$

$q_{b}^{*}=\frac{w_{b}}{\sum_{b=1}^{h} w_{b}}$ is the weighted share of all clusters in block $b$, and $v^{*}$ is a degrees of freedom adjustment for the covariates. As discussed in Donald and Lang (2007), plausible values for $v^{*}$ are $v^{*}=v$ (the number of covariates), which applies when using cluster-level covariates, or $v^{*}=0$, which applies when using individual-level covariates that vary only within clusters and 
not between clusters. Other approaches have been proposed, such as adjusting $v$ for design effects due to clustering (Hedges 2007) and minimum distance methods (Wooldridge 2006).

In our simulations in Section 6, we also use two variants of (9). First, we multiply (9) by $\left(1-R_{T X b}^{2}\right)^{-1}$, where $R_{T X b}^{2}$ is the R-squared value from a regression of $S_{i j b, s} \tilde{T}_{j b}$ on $\tilde{\mathbf{x}}_{i j b}$ and the other block-by-treatment status interactions in (5) (with no intercept). This term captures the finite sample collinearity between $\tilde{T}_{j b}$ and $\tilde{\mathbf{x}}_{i j b}$ (which inflates the variances). This estimator performs well in our simulations. The second variant subtracts $\frac{1}{m_{b}}\left(\sqrt{s_{D_{b}}^{2}(1)}-\sqrt{s_{D_{b}}^{2}(0)}\right)^{2}$, a lower bound on the finite population heterogeneity term based on the Cauchy-Schwarz inequality. Aronow et al. (2014) discuss sharper bounds on this heterogeneity term by approximating the marginal distributions of potential outcomes.

The regression model can also be estimated using data averaged to the cluster level, so that setting $v^{*}=v$ yields well-defined degrees of freedom. For models that use cluster-level covariates only (or no covariates), estimators using the individual and aggregate data will coincide. However, for models with individual-level covariates that vary both within and between clusters, the estimators can differ. In this case, as discussed in Section 4.1, data aggregation yields asymptotically efficient estimators. However, as proved and quantified in Section A.6 in the supplementary materials, in finite samples, precision losses from using individual data could be offset by precision gains due to the reduced collinearity between the covariates and treatment indicators ( $T X$ collinearity). In our simulations, we examine precision levels using the individual and aggregate data to assess these counteracting factors.

Finally, we can obtain pooled ATE estimators across all blocks by inserting $\hat{\beta}_{1, b}$ into (2) and using $\frac{1}{(h \bar{w})^{2}} \sum_{b=1}^{h} w_{b}^{2} \operatorname{Vâr}\left(\widehat{D}_{b}\right)$ for variance estimation. Hypothesis testing can be conducted using 
z-tests. Alternatively, results in Bell and McCaffery (2002), Hansen (2007), and Cameron and Miller (2015) for the CRSE estimator suggest that t-tests with $\left(m-2 h-v^{*}\right)$ degrees of freedom perform better in small samples and is what we use hereafter.

\subsection{Comparing Design-Based and CRSE Estimators}

The CRSE variance estimator is an extension of robust standard errors (Huber 1967; White 1980) to clustered designs (Liang and Zeger 1986). The CRSE approach, which assumes iid sampling of clusters from some (infinite) super-population, allows for errors to be correlated within clusters but not across clusters. As with the design-based estimators, the CRSE estimator is based on weighted least squares using the pooled data across blocks and is asymptotically normal. Therefore, both approaches yield the same ATE estimator when the covariates and weights are the same, but the variance estimators differ in several ways.

To illustrate the key variance differences, consider first the model without covariates. In this case, as shown in Section A.7 in the supplementary materials, the CRSE variance estimator for a single block-by-treatment ATE estimate, $\hat{\beta}_{1, b}$, is

$$
\operatorname{Vâr}_{C R S E}\left(\hat{\beta}_{1, b}\right)=g \frac{s_{D_{b}}^{2 *}(1)}{m_{b}^{1}}+g \frac{s_{D_{b}}^{2 *}(0)}{m_{b}^{0}}
$$

where $s_{D_{b}}^{2 *}(1)=\frac{\left(m_{b}^{1}-1\right)}{m_{b}^{1}} s_{D_{b}}^{2}(1), s_{D_{b}}^{2 *}(0)=\frac{\left(m_{b}^{0}-1\right)}{m_{b}^{0}} s_{D_{b}}^{2}(0)$, and $g$ is a small sample correction term.

Here, we use $g=\left(\frac{m}{m-1}\right)\left(\frac{n-1}{n-2 h-v}\right)$, a common value in statistical software packages such as Stata (Cameron and Miller 2015), although other approaches have been proposed, such as biascorrected CRSE estimators (Mackinnon and White 1985; Bell and McCaffery 2002; Angrist and Lavy 2002; Pustejovsky and Tipton 2018) and bootstrap methods (Cameron et al. 2008; Webb 2013) to adjust for the known Type 1 error inflation of the CRSE estimator in small samples.

Compare (10) to the following parallel expression for the design-based estimator in (9): 


$$
\operatorname{Vâr}\left(\hat{D}_{b}\right)=\frac{m_{b}^{1}}{\left(m_{b}^{1}-1\right)} \frac{s_{D_{b}}^{2 *}(1)}{m_{b}^{1}}+\frac{m_{b}^{0}}{\left(m_{b}^{0}-1\right)} \frac{s_{D_{b}}^{2 *}(0)}{m_{b}^{0}} \text {. }
$$

Examining (10) and (11) establishes that the design-based and CRSE variance estimators are asymptotically equivalent, because both correction terms converge to 1 as $m_{b}^{1} \rightarrow \infty$ and $m_{b}^{0} \rightarrow$ $\infty$. In finite samples, however, there are two key differences between the estimators that pertain to the degrees of freedom adjustments. First, the adjustments for the design-based variance estimator are applied separately for treatments and controls based on $m_{b}^{1}$ and $m_{b}^{0}$, whereas the standard CRSE estimator applies a single adjustment, $g$, based on total sample sizes ( $m$ and $n$ ). Second, the design-based estimator uses $(m-2)$ degrees of freedom for the t-tests, reflecting separation of the two research groups, whereas the CRSE estimator commonly uses $(m-1)$ (Cameron and Miller 2015). These two differences will typically lead to larger design-based variances and lower rejection rates. Note also that the finite population heterogeneity term does not apply to the CRSE estimator as it assumes a super-population sampling framework. Similar results apply to the model with covariates (see Section A.7 in the supplementary materials).

\section{Restricted ATE Estimators with Fixed Block Effects Only}

A commonly used estimation strategy for blocked designs is to include block indicator variables in the regression model but to exclude block-by-treatment status interaction terms:

$$
y_{i j b}=\beta_{1, R} \tilde{T}_{j b}+\sum_{s=1}^{h} \delta_{0, s} S_{i j b, s}+\epsilon_{i j b}
$$

where $\epsilon_{i j b}$ is the error term. Because this framework imposes restrictions on the assumed data structure, it typically produces asymptotically biased estimates of the true ATE parameter in (2). Nevertheless, it has practical appeal due to its parsimony and additional degrees of freedom. 
Consider WLS estimation of (12) where the model includes the $\tilde{\mathbf{x}}_{i j b}$ covariates with parameter vector $\boldsymbol{\gamma}$. As shown in Section A.4 of the supplementary materials, the WLS estimator for $\beta_{1, R}$ is a weighted average of block-level ATE estimates with weights, $\widetilde{w}_{b, R}=\frac{1}{m w_{b}} w_{b}^{0} w_{b}^{1}$ :

$$
\hat{\beta}_{1, R}=\sum_{b=1}^{h} \frac{\widetilde{w}_{b, R}}{\sum_{a=1}^{h} \widetilde{w}_{a, R}}\left(\overline{\bar{y}}_{b}(1)-\overline{\bar{y}}_{b}(0)-\left(\overline{\bar{x}}_{b}^{1}-\overline{\overline{\boldsymbol{x}}}_{b}^{0}\right) \hat{\boldsymbol{\gamma}}\right)
$$

The weights can also be expressed in the limit as $\widetilde{w}_{b, R}=q_{b} p_{b}\left(1-p_{b}\right) \bar{w}_{b}$, where $q_{b}=m_{b} / m$. Thus, this approach uses a form of precision weighting to weight the block-specific treatment effects and is analogous to a fixed effects regression model using non-clustered data. The weights for the restricted model will differ from those for the unrestricted model (causing asymptotic bias), except if the ATEs or $p_{b}\left(1-p_{b}\right)$ values are homogenous across blocks.

\subsection{Theoretical Results}

We now present a CLT for $\hat{\beta}_{1, R}$ that is proved in Section A.4 in the supplementary materials. Let

$$
\beta_{1, R}=\sum_{b=1}^{h} \frac{q_{b} p_{b}\left(1-p_{b}\right) \bar{w}_{b}}{\sum_{b=1}^{h} q_{b} p_{b}\left(1-p_{b}\right) \bar{w}_{b}}\left(\overline{\bar{Y}}_{b}(1)-\overline{\bar{Y}}_{b}(0)\right)
$$

denote the treatment effect parameter for the restricted model. Also define the vector of blocklevel estimators as a series of triples:

$$
\mathbf{t}=\left(\bar{w}_{1}^{1}\left(\overline{\bar{y}}_{1}(1)-\overline{\mathbf{x}}_{1}^{1} \boldsymbol{\gamma}\right), \bar{w}_{1}^{0}\left(\overline{\bar{y}}_{1}(0)-\overline{\mathbf{x}}_{1}^{0} \boldsymbol{\gamma}\right), \bar{w}_{1}^{1}, \cdots, \bar{w}_{1}^{h}\left(\overline{\bar{y}}_{h}(1)-\overline{\mathbf{x}}_{h}^{1} \boldsymbol{\gamma}\right), \bar{w}_{h}^{0}\left(\overline{\bar{y}}_{h}(0)-\overline{\mathbf{x}}_{h}^{0} \boldsymbol{\gamma}\right), \bar{w}_{h}^{1}\right) .
$$

Theorem 2. Assume $(C 1),(C 2),(C 4)$ for $f_{b}^{t},(C 5),(C 6),(C 7)$ and the following conditions for $t \in\{1,0\}$ :

(C8) As $m \rightarrow \infty$,

$$
\begin{gathered}
\max _{1 \leq b \leq h} \frac{a_{Y, b}(t)}{p_{b}\left(1-p_{b}\right) m_{b} v_{Y, b}(t)} \rightarrow 0 \text {, where } \\
a_{Y, b}(t)=\max _{1 \leq j \leq m_{b}}\left(w_{j b}\left(\bar{Y}_{j b}(t)-\overline{\mathbf{x}}_{j b} \boldsymbol{\gamma}\right)-\bar{w}_{b}\left(\overline{\bar{Y}}_{b}(t)-\overline{\overline{\mathbf{x}}}_{b} \boldsymbol{\gamma}\right)\right)^{2} \text { and }
\end{gathered}
$$




$$
v_{Y, b}(t)=\frac{1}{m_{b}-1} \sum_{j=1}^{m_{b}}\left(w_{j b}\left(\bar{Y}_{j b}(t)-\overline{\mathbf{x}}_{j b} \boldsymbol{\gamma}\right)-\bar{w}_{b}\left(\overline{\bar{Y}}_{b}(t)-\overline{\overline{\mathbf{x}}}_{b} \boldsymbol{\gamma}\right)\right)^{2}
$$

(C9) As $m \rightarrow \infty$,

$$
\max _{1 \leq b \leq h} \frac{a_{w, b}}{p_{b}\left(1-p_{b}\right) m_{b} v_{w, b}} \rightarrow 0
$$

where $a_{w, b}=\max _{1 \leq j \leq m_{b}}\left(w_{j b}-\bar{w}_{b}\right)^{2}$ and $v_{w, b}=\frac{1}{m_{b}-1} \sum_{j=1}^{m_{b}}\left(w_{j b}-\bar{w}_{b}\right)^{2}$.

(C10) The correlation matrix of $\mathbf{t}$ has a finite limiting value $\boldsymbol{\Sigma}$.

(C11) The variance expressions, $v_{w, b}$ and $v_{Y, b}(t)$, have finite limiting values for $b \in\{1, \ldots, h\}$.

(C12) $\bar{w}_{b}\left(\overline{\bar{Y}}_{b}(1)-\overline{\overline{\mathbf{x}}}_{b} \boldsymbol{\gamma}\right) \neq 0$ or $\bar{w}_{b}\left(\overline{\bar{Y}}_{b}(0)-\overline{\overline{\mathbf{x}}}_{b} \boldsymbol{\gamma}\right) \neq 0$ for some $b$

Then, as $m \rightarrow \infty, \hat{\beta}_{1, R}$ is a consistent estimator for $\beta_{1, R}$ and

$$
\frac{\hat{\beta}_{1, R}-\beta_{1, R}}{\sqrt{\operatorname{Var}\left(\widetilde{\beta}_{1, R}\right)}} \stackrel{d}{\rightarrow} N(0,1), \text { where }
$$

$\operatorname{Var}\left(\widetilde{\beta}_{1, R}\right)$

$$
\begin{aligned}
& =\sum_{b=1}^{h} \frac{1}{m_{b}\left(m_{b}-1\right)} \frac{\left(q_{b} p_{b}\left(1-p_{b}\right) \bar{w}_{b}\right)^{2}}{\left(\sum_{a=1}^{h} q_{a} p_{a}\left(1-p_{a}\right) \bar{w}_{a}\right)^{2}} \sum_{j=1}^{m_{b}}\left(\sqrt{\frac{1-p_{b}}{p_{b}}}\left(\frac{w_{j b}\left(\bar{Y}_{j b}(1)-\overline{\bar{Y}}_{b}(1)-\left(\overline{\mathbf{x}}_{j b}-\overline{\mathbf{x}}_{b}\right) \boldsymbol{\gamma}\right)}{\bar{w}_{b}}\right)\right. \\
& +\sqrt{\frac{p_{b}}{1-p_{b}}}\left(\frac{w_{j b}\left(\bar{Y}_{j b}(0)-\overline{\bar{Y}}_{b}(0)-\left(\overline{\mathbf{x}}_{j b}-\overline{\overline{\mathbf{x}}}_{b}\right) \boldsymbol{\gamma}\right)}{\bar{w}_{b}}\right) \\
& \left.+\frac{\left(1-2 p_{b}\right)}{\sqrt{p_{b}\left(1-p_{b}\right)} \bar{w}_{b}}\left(\beta_{1, b}-\beta_{1, R}\right)\left(w_{j b}-\bar{w}_{b}\right)\right)^{2} .
\end{aligned}
$$

Remark. The first two terms inside the brackets in (14) pertain to block-specific variances for the treatment and control groups that are analogous to the corresponding variance terms in the unrestricted model in (8). The third term represents the covariance between the block treatment effects and block weights that is induced by the restricted model. This term differentiates the 
variances for the restricted and unrestricted models, along with the block weights used for pooling and the presence of the finite population heterogeneity term. This third term is 0 if $p_{b}=$ 0.5 or $\operatorname{Cov}\left(\beta_{1, b}, w_{j b}\right)=0$ for all $b$, but otherwise can be positive or negative.

\subsection{Variance Estimation}

A consistent variance estimator for (14) can be obtained by multiplying out the squared term and using plug-in estimators for each of the resulting six terms. However, it is simpler to use the following consistent estimator based on cluster-level model residuals:

$$
\operatorname{Vâr}\left(\hat{\beta}_{1, R}\right)=\frac{m}{\left(m-h-v^{*}-1\right)} \frac{\sum_{b=1}^{h} \sum_{j=1}^{m_{b}} w_{j b}^{2} \tilde{T}_{j b}^{2}\left(\bar{y}_{j b}-\hat{\beta}_{1, R} \tilde{T}_{j b}-\hat{\delta}_{0, b}-\tilde{\overline{\mathbf{x}}}_{j b} \hat{\boldsymbol{\gamma}}\right)^{2}}{\left(\sum_{b=1}^{h} m_{b} p_{b}^{*}\left(1-p_{b}^{*}\right) \bar{w}_{b}\right)^{2}},
$$

recalling that $\tilde{T}_{j b}=\left(T_{j b}-p_{b}^{*}\right)$. Following Schochet (2016), the expression in (15) can be justified using the following standard asymptotic expansion for the WLS estimator:

$$
\sqrt{m}\left(\hat{\beta}_{1, R}-\beta_{1, R}\right)=\frac{\sum_{b=1}^{h} \sum_{j=1}^{m_{b}} w_{j b} \tilde{T}_{j b}\left(\bar{y}_{j b}-\beta_{1, R} \tilde{T}_{j b}-\delta_{0, b}-\tilde{\mathbf{x}}_{j b} \boldsymbol{\gamma}\right)}{\sqrt{m} \sum_{b=1}^{h} q_{b} p_{b}\left(1-p_{b}\right) \bar{w}_{b}}+o_{p}(1)
$$

where $o_{p}(1)$ signifies a term that converges in probability to zero. Suppose we insert into (16),

$\bar{y}_{j b}=T_{b j} \bar{Y}_{j b}(1)+\left(1-T_{b j}\right) \bar{Y}_{j b}(0)$ and $\delta_{0, b}=p_{b} \overline{\bar{Y}}_{b}(1)+\left(1-p_{b}\right) \overline{\bar{Y}}_{b}(0)$ (see Section A.4 in the supplement), and then add and subtract $\left(\beta_{1, b}-\beta_{1, R}\right) \widetilde{T}_{j b}$. If we then calculate $\operatorname{Var}\left(\hat{\beta}_{1, R}\right)$ over the randomization distribution, we obtain (15) after some algebra. Hypothesis testing can be conducted using t-tests with $\left(\sum_{b=1}^{h}\left(m_{b}^{1}+m_{b}^{0}\right)-v^{*}-h-1\right)$ degrees of freedom. Note that with $v^{*}=0$, estimation only requires at least 1 treatment and 1 control cluster per block rather than two as for the fully interacted model. A $\left(1-R_{T X}^{2}\right)^{-1}$ correction can also be applied to (15).

\section{Simulation Results}

To examine the statistical properties of the design-based estimator, we conducted simulations for a clustered, non-blocked design $(h=1)$. We applied the variance estimator in (9) with and 
without (1) model covariates, (2) the $R_{T X}^{2}$ adjustment, and (3) the finite population heterogeneity term based on the Cauchy-Schwarz inequality. We also included the standard CRSE estimator to identify sources of differences between the two approaches (our goal is not to compare various proposed CRSE estimators to each other). Note that if $h>1$, differences in block-level estimates between the two approaches will be greater than the simulation results presented here (holding $m$ fixed), because the degrees of freedom adjustments will differ more (see (10) and (11)).

To generate potential outcomes for our primary simulations, we used the following model (see Section B in the supplementary materials for simulation details):

$$
\begin{gathered}
Y_{i j}(0)=x_{i j 1}+x_{i j 2}+u_{j}+e_{i j} \\
Y_{i j}(1)=Y_{i j}(0)+\theta_{j}
\end{gathered}
$$

where $u_{j}, \theta_{j}$ (which captures treatment effect heterogeneity), and $e_{i j}$ are each iid mean zero random errors and $x_{i j 1}$ and $x_{i j 2}$ are independent covariates. For each draw of potential outcomes, we conducted 1,000 replications where we randomly assigned clusters to either the treatment or control group. We ran separate simulations for $m=8$ to 50 clusters.

We examined a range of simulation scenarios for the covariates and model distributions. We generated data with (1) no covariates (excluding $x_{i j 1}$ and $x_{i j 2}$ from (17)); (2) two individuallevel covariates (applying an intraclass correlation coefficient of $\rho_{X}=0$ ); (3) two cluster-level covariates (applying $\rho_{X}=1$ ); and (4) one individual-level and one cluster-level covariate. For the models with individual-level covariates, we calculated the degrees of freedom in three ways, setting $v^{*}$ equal to 0 , the total number of covariates $(v)$, or the number of cluster-level covariates (if applicable). We generated data assuming normal, bimodal, and chi-square distributions for the errors and covariates in (17). 
Finally, to compare the true variances of models estimated using individual and aggregate data (see Sections 4.1 and 4.2), rather than directly generating data for $x_{i j k}$, we instead generated data separately for the between- and within-cluster components, $\bar{x}_{j k}$ and $\left(x_{i j k}-\bar{x}_{j k}\right)$. We then included these components as covariates in (17) to generate the outcomes, allowing for different parameter values, $\gamma_{B k}$ and $\gamma_{W k}$. The individual-level models, however, were estimated using $x_{i j k}$ only (not the components) with parameter $\gamma_{k}$ (see the supplementary materials for details).

Results without covariates. For models without covariates, the simulation results indicate that the design-based estimator without the correction for the finite population heterogeneity term yields Type 1 errors near the 5 percent nominal level and standard errors near true values, even with relatively few clusters (see Figure 1 and Table B.1 for the full results). In contrast, the standard CRSE estimator in (10) yields inflated Type 1 errors similar to those found in the literature using a super-population simulation framework (see, e.g., Cameron et al. 2008; Green and Vavreck 2008; Angrist and Pischke 2009) (Figure 1; Table B.1). These differences arise because the CRSE estimator applies a single degrees of freedom variance adjustment based on the total sample size, whereas the design-based estimator applies a separate adjustment for the treatment and control groups, which inflates the variances. A more minor reason is that the CRSE approach uses $(m-1)$ degrees of freedom for the t-tests rather than $(m-2)$. The design-based variance estimator that includes a correction for the finite population heterogeneity term also overrejects (so we do not focus on this estimator in what follows), but less so than the CRSE estimator (Figure 1; Table B.4). We find similar simulation results using different model distributions (Table B.1) and individual sample sizes (Table B.4).

Biases of the ATE estimators are negligible (Table B.1). Further, mean squared errors of the estimated standard errors are nearly identical for the two approaches, suggesting similar stability 
in estimating uncertainty (Table B.1). This result occurs because the larger variance of the estimated standard errors for the design-based estimator is offset by the smaller bias in its estimated standard errors (relative to the "true" values as measured by the standard deviation of the 1,000 ATE estimates across replications). Finally, for small $m$, power levels are lower for the design-based than CRSE estimator (due to lower Type 1 errors), but the design-based estimator more closely matches power levels calculated using the true standard errors (Table B.2).

Results with covariates. A similar pattern of results arises when covariates are included in the model (and standard errors decrease) (Figure 2; Tables B.3 and B.4). For the model with individual-level covariates only $\left(\rho_{X}=0\right)$, we find that the design-based estimator with $v^{*}=0$ yields Type 1 errors near the nominal level (Model (1) in Figure 2). In this case, the $R_{T X}^{2}$ adjustment has little effect on the results (Table B.3). If we instead apply $v^{*}=2$, the design-based approach becomes conservative (Model (2) in Figure 2). As before, the standard CRSE estimator yields inflated Type 1 errors (Model (3) in Figure 2).

For the model with cluster-level covariates only $\left(\rho_{X}=1 ; v^{*}=2\right)$, which is identical to aggregating the data to the cluster level, the design-based estimator yields Type 1 errors at the nominal level if the $R_{T X}^{2}$ adjustment is applied, even with $m=8$, but overrejects without this adjustment (Models (4) and (5) in Figure 2). For this specification, the CRSE estimator produces Type 1 errors that are more inflated than with individual covariates (Model (6) in Figure 2).

For models containing one individual-level covariate $\left(v_{1}=1\right)$ and one cluster-level covariate $\left(v_{2}=1\right)$, we find that for the design-based estimator, setting $v^{*}=0$ is liberal; setting $v^{*}=v_{2}=1$ yields Type 1 errors close to the nominal rate; and setting $v^{*}=v_{1}+v_{2}=2$ is conservative (Table B.4). As before, the CRSE tends to overreject with few clusters. 
Finally, the simulation results comparing the true variances based on the individual and aggregate data - where we generated data using $\bar{x}_{j k}$ and $\left(x_{i j k}-\bar{x}_{j k}\right)$ but estimated the models using $x_{i j k}$ only—support the theory presented in Sections 4.1 and 4.2 (Table B.5). When $\gamma_{B k}=$ $\gamma_{W k}$, the true variances are always smaller using the individual data because $\gamma_{k}$ is asymptotically efficient and the $T X$ collinearities are always smaller. The differences decrease, however, as $m$ increases and the $T X$ collinearities become negligible. In contrast, when $\gamma_{B k} / \gamma_{W k}=2$, with a single covariate, the aggregate data produce more precise estimates, even when $m=8$. However, with five covariates, the $T X$ collinearities become more problematic, so even when $\gamma_{B k} / \gamma_{W k}=2$, the individual data yield efficiency gains unless $m \geq 50$. With more covariates, the $T X$ collinearities using the aggregate data are severe, favoring the use of the individual data.

Super-population simulation results. To compare our results to those in the literature for the CRSE estimator, we also conducted select simulations using a super-population framework by generating 50,000 separate datasets. These results show a similar pattern to the results above, but with somewhat larger true standard errors (Table B.6).

Discussion. Overall, the simulation results suggest that the design-based estimator has beneficial statistical properties with few clusters for models with or without covariates. For models with covariates and relatively large $n$ (typical for clustered RCTs in practice), the results suggest that setting $v^{*}=v_{2}$ (the number of cluster-level covariates) could be a good general strategy, and that setting $v^{*}=v$ (the total number of covariates) is conservative. The simulations further indicate that even with individual-level covariates, the design-based estimator using data averaged to the cluster-level with the $R_{T X}^{2}$ adjustment and $v^{*}=v$ yields nominal rejection rates. While aggregation can result in losses in statistical power (due to increased $T X$ collinearities), it could be a good strategy with small numbers of covariates if $m$ is moderate. 


\section{Empirical Application Using the Motivating SACD Example}

To demonstrate the considered estimators, we use outcome and baseline data from the SACD evaluation on 4,018 4th graders (2,147 treatments and 1,871 controls) in 84 schools in 7 large school districts. The data were obtained from student reports administered in the classroom, primary caregiver telephone interviews, and teacher reports on students (SACD Consortium, 2010). We analyze six primary study outcome scales (Table 1) and adjust for baseline covariates selected in an initial step from the 46 available, along with their two-way interactions, using Least Absolute Shrinkage and Selection Operator (LASSO) methods with 5-fold cross-validation (Tibshirani 1996). We use the LASSO-WLS hybrid procedure for clustered RCTs developed in Schochet (2020b) based on the design-based estimators presented above (see also Bloniarz et al. 2016). In the first stage, LASSO estimation is conducted using cluster-level averages, and in the second stage, design-based WLS estimation is conducted using the individual data and first stage LASSO covariates. Our goal is not to replicate study results but to illustrate the ATE estimators.

Table 2 presents the estimation results for various model specifications: (1) with and without baseline covariates, (2) with and without block-by-treatment status interaction terms, and (3) with equal weighting of individuals (to estimate ATEs for the average student in the sample) versus equal weighting of sites and clusters (to estimate ATEs for the average school in the average district). Our methods can easily accommodate weights to adjust for data item nonresponse. We present results for both the design-based and standard CRSE estimators.

The results indicate that for all specifications, the behavioral health interventions had no statistically significant effect on any outcome scale, although the negative estimate on the scale measuring fear in school is marginally statistically significant at the 10 percent level for most models with covariates (Table 2). Across the six outcomes, standard errors are about 16 to 35 
percent smaller when covariates are included in the models. Further, we find very similar results for the fully-interacted and restricted models for two reasons: (1) the estimated treatment effects vary little across sites (an average standard deviation of 0.07 across the outcomes) and (2) the two sets of site weights are highly correlated (greater than 0.95 ) because $p_{b}$ is about 0.5 in all sites. Further, because sample sizes do not vary substantially across sites (they range from 425 to 650 students and 10 to 14 schools), findings do not materially differ when individuals versus blocks and clusters are weighted equally, although in the latter case, standard errors increase due to design effects from weighting and the marginally significant impact on the scale measuring fear at school disappears. Finally, consistent with the theory and simulations, standard errors are somewhat larger using the design-based estimators than the parallel CRSE estimators.

\section{Conclusions}

This article considered design-based ratio estimators for clustered, blocked RCTs using the Neyman-Rubin-Holland model and weighted least squares methods. We developed finite population CLTs for the ATE estimators, allowing for baseline covariates to improve precision, general weighting schemes, and several common approaches for handling blocks in the models. We showed that the design-based ratio estimators are attractive in that they yield consistent and asymptotically normal ATE estimators with simple variance estimators based on cluster-level model residuals; apply to continuous, binary, and discrete outcomes; and yield Type 1 errors at nominal levels for models with and without covariates, even in small samples. Our theory applies to analyses conducted using either individual or aggregate (cluster-level) data, where our results suggest that in practice, the use of individual data will tend to yield more precise estimates for models with covariates (that vary both within and between clusters), unless the number of covariates is very small. 
An unexpected finding is that the "conservative" variance estimator that excludes a correction for the finite population heterogeneity term based on the Cauchy-Schwarz inequality improves statistical performance. Further, for models with covariates, an $R_{T X}^{2}$ adjustment for the collinearity between the covariates and treatment indicator improves results in designs with few clusters and subjects.

Our findings justify the CRSE estimator from a finite population perspective (even though it estimates a super-population ATE parameter); this contribution follows similar literature for the individual randomized case (see, e.g., Freedman 2008 and Lin 2013). However, while the structure of the design-based and standard CRSE variance estimators are similar, differences in their degrees of freedom adjustments do affect their statistical performance in small samples (the standard CRSE estimator overrejects in this case). The key difference is simple: the randomization mechanism leads to separate degrees of freedom adjustments for the treatment and control groups based on their respective numbers of clusters (in each block), whereas the CRSE approach often used in practice applies a single adjustment based on the total number of clusters. These differences tend to increase with more blocks.

As discussed in the article, other corrections for the CRSE estimator have been proposed that can improve the Type 1 error inflation rate in general settings. In the RCT setting, however, the advantage of the design-based variance estimator is that it is tailored to experiments, as it is derived directly from principles underlying them. Further, it is simple to apply and parallels design-based estimators for non-clustered RCTs. The free RCT-YES software (www.rctyes.com), funded by the U.S. Department of Education, estimates ATEs for full sample and baseline subgroup analyses using the design-based methods discussed in this article using either $\mathrm{R}$ or Stata, and also allows for multi-armed trials with multiple treatment conditions. 


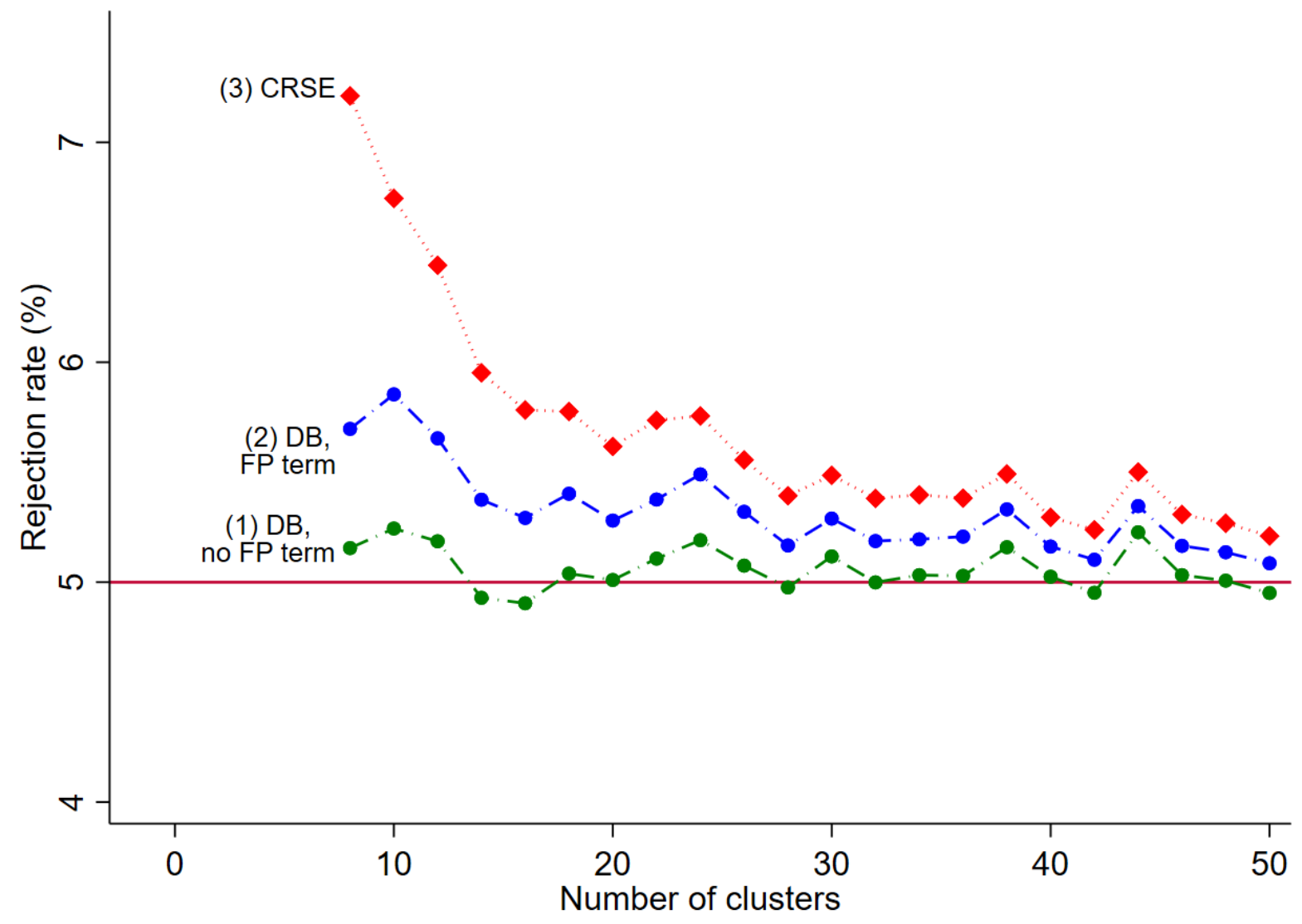

Figure 1. Type I Error Rates for Models without Covariates

Abbreviations. DB = Design-based variance estimator; FP term = Finite population heterogeneity term included based on the Cauchy-Schwarz inequality; CRSE = Standard cluster-robust standard error estimator. 


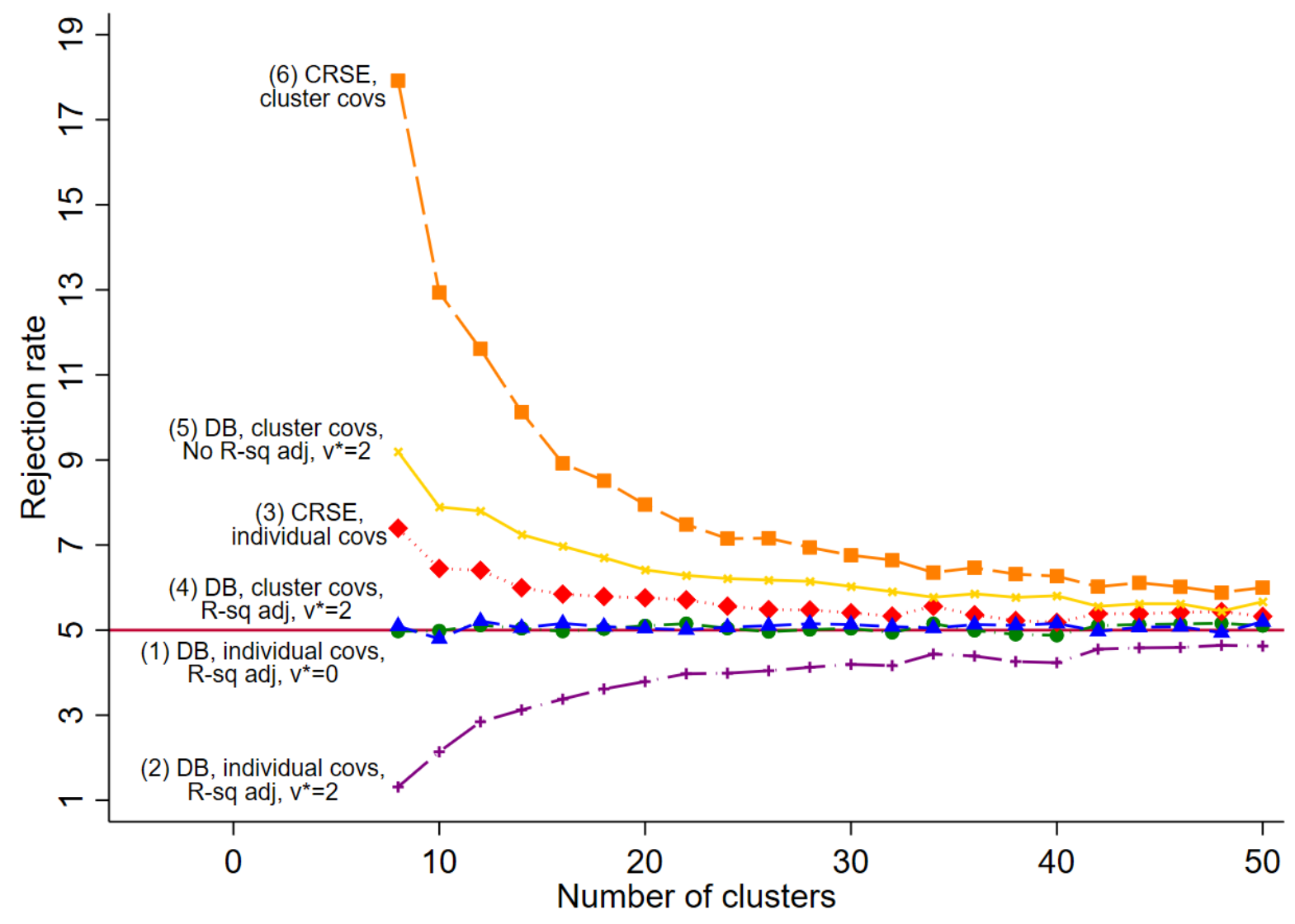

Figure 2. Type I Error Rates for Models with Covariates

Abbreviations. DB = Design-based variance estimator in (9) (without the finite population heterogeneity term); "cluster covs" = Two cluster-level covariates included in the model; "Individual covs" = Two individual-level covariates included in the model; "R-sq-adj" $=R_{T X}^{2}$ adjustment applied to the DB estimator; $v^{*}=$ Degrees of freedom adjustment for the covariates for the DB estimator; CRSE = Standard cluster-robust standard error estimator. 
Table 1. Outcome variables for the empirical analysis using SACD RCT data.

\begin{tabular}{|c|c|c|}
\hline Outcome & $\begin{array}{l}\text { Data source } \\
\text { (Spring 2007) }\end{array}$ & Description of variable \\
\hline Problem behavior & Child report & $\begin{array}{l}\text { Scale ranges from } 0 \text { to } 3 \text { and contains } 6 \text { items from the } \\
\text { Frequency of Delinquent Behavior Scale and } 6 \text { items from the } \\
\text { Aggression Scale; Reliability }=0.86 \text {. }\end{array}$ \\
\hline $\begin{array}{l}\text { Normative beliefs } \\
\text { about aggression }\end{array}$ & Child report & $\begin{array}{l}\text { Scale ranges from } 1 \text { to } 4 \text { and contains } 12 \text { items from the } \\
\text { Normative Beliefs About Aggression Scale; Reliability }=0.83 \text {. }\end{array}$ \\
\hline $\begin{array}{l}\text { Student afraid at } \\
\text { school }\end{array}$ & Child report & $\begin{array}{l}\text { Scale ranges from } 1 \text { to } 4 \text { and contains } 4 \text { items from the } \\
\text { Feelings of Safety at School scale; Reliability }=0.79 \text {. }\end{array}$ \\
\hline Altruistic behavior & $\begin{array}{l}\text { Primary caregiver } \\
\text { report }\end{array}$ & $\begin{array}{l}\text { Scale ranges from } 1 \text { to } 4 \text { and contains } 8 \text { items from the } \\
\text { Altruism Scale, Primary Caregiver Version; Reliability }=0.88\end{array}$ \\
\hline $\begin{array}{l}\text { Positive social } \\
\text { behavior }\end{array}$ & Teacher report & $\begin{array}{l}\text { Scale ranges from } 1 \text { to } 4 \text { and contains } 6 \text { items from the } \\
\text { Responsibility Scale and } 19 \text { items from the Social Competence } \\
\text { Scale and } 8 \text { items from the Altruism Scale, Teacher Version; } \\
\text { Reliability }=0.97 \text {. }\end{array}$ \\
\hline Problem behavior & Teacher report & $\begin{array}{l}\text { Scale ranges from } 1 \text { to } 4 \text { and contains } 14 \text { items from the BASC } \\
\text { Aggression Subscale, Teacher Version, } 7 \text { items from the BASC } \\
\text { Conduct Problems Subscale, Teacher Version and } 2 \text { items from } \\
\text { the Responsibility Scale; Reliability }=0.95 \text {. }\end{array}$ \\
\hline
\end{tabular}

Note: See SACD Research Consortium (2010) for a complete description of the construction of these scales. 
Table 2. Estimated ATEs and standard errors for the SACD study, by model specification.

\begin{tabular}{|c|c|c|c|c|c|c|}
\hline \multirow[b]{3}{*}{$\begin{array}{l}\text { Outcome variable and } \\
\text { covariate specification }\end{array}$} & \multicolumn{4}{|c|}{$\begin{array}{l}\text { Model with site-by-treatment } \\
\text { interaction terms }\end{array}$} & & \\
\hline & \multicolumn{2}{|c|}{$\begin{array}{c}\text { Individuals } \\
\text { weighted equally }\end{array}$} & \multicolumn{2}{|c|}{$\begin{array}{l}\text { Schools and sites } \\
\text { weighted equally }\end{array}$} & \multicolumn{2}{|c|}{$\begin{array}{l}\text { Model with site } \\
\text { fixed effects only }\end{array}$} \\
\hline & $\begin{array}{l}\text { Design- } \\
\text { based }\end{array}$ & $\begin{array}{l}\text { Standard } \\
\text { CRSE }\end{array}$ & $\begin{array}{l}\text { Design- } \\
\text { based }\end{array}$ & $\begin{array}{l}\text { Standard } \\
\text { CRSE }\end{array}$ & $\begin{array}{l}\text { Design- } \\
\text { based }\end{array}$ & $\begin{array}{l}\text { Standard } \\
\text { CRSE }\end{array}$ \\
\hline \multicolumn{7}{|l|}{$\begin{array}{l}\text { Model without } \\
\text { covariates }\end{array}$} \\
\hline $\begin{array}{l}\text { Problem behavior } \\
\text { (CR) }\end{array}$ & $\begin{array}{c}0.006 \\
(0.037)\end{array}$ & $\begin{array}{c}0.006 \\
(0.034)\end{array}$ & $\begin{array}{c}0.011 \\
(0.041)\end{array}$ & $\begin{array}{c}0.011 \\
(0.038)\end{array}$ & $\begin{array}{c}0.006 \\
(0.036)\end{array}$ & $\begin{array}{c}0.006 \\
(0.035)\end{array}$ \\
\hline $\begin{array}{l}\text { Normative beliefs about } \\
\text { aggression (CR) }\end{array}$ & $\begin{array}{c}0.003 \\
(0.031)\end{array}$ & $\begin{array}{c}0.003 \\
(0.029)\end{array}$ & $\begin{array}{c}0.000 \\
(0.038)\end{array}$ & $\begin{array}{c}0.000 \\
(0.035)\end{array}$ & $\begin{array}{c}0.003 \\
(0.031)\end{array}$ & $\begin{array}{c}0.003 \\
(0.030)\end{array}$ \\
\hline $\begin{array}{l}\text { Student afraid at school } \\
\text { (CR) }\end{array}$ & $\begin{array}{l}-0.064 \\
(0.052)\end{array}$ & $\begin{array}{l}-0.064 \\
(0.048)\end{array}$ & $\begin{array}{l}-0.041 \\
(0.061)\end{array}$ & $\begin{array}{l}-0.041 \\
(0.056)\end{array}$ & $\begin{array}{l}-0.064 \\
(0.050)\end{array}$ & $\begin{array}{l}-0.064 \\
(0.048)\end{array}$ \\
\hline $\begin{array}{l}\text { Altruistic behavior } \\
(\mathrm{PCR})\end{array}$ & $\begin{array}{l}-0.006 \\
(0.035)\end{array}$ & $\begin{array}{l}-0.006 \\
(0.032)\end{array}$ & $\begin{array}{l}-0.011 \\
(0.041)\end{array}$ & $\begin{array}{l}-0.011 \\
(0.037)\end{array}$ & $\begin{array}{l}-0.006 \\
(0.034)\end{array}$ & $\begin{array}{l}-0.006 \\
(0.033)\end{array}$ \\
\hline $\begin{array}{l}\text { Positive social behavior } \\
\text { (TR) }\end{array}$ & $\begin{array}{l}-0.046 \\
(0.061)\end{array}$ & $\begin{array}{l}-0.046 \\
(0.056)\end{array}$ & $\begin{array}{l}-0.036 \\
(0.065)\end{array}$ & $\begin{array}{l}-0.036 \\
(0.060)\end{array}$ & $\begin{array}{l}-0.045 \\
(0.060)\end{array}$ & $\begin{array}{l}-0.045 \\
(0.056)\end{array}$ \\
\hline $\begin{array}{l}\text { Problem behavior } \\
\text { (TR) }\end{array}$ & $\begin{array}{c}0.019 \\
(0.040)\end{array}$ & $\begin{array}{c}0.019 \\
(0.036)\end{array}$ & $\begin{array}{c}0.006 \\
(0.044)\end{array}$ & $\begin{array}{c}0.006 \\
(0.040)\end{array}$ & $\begin{array}{c}0.019 \\
(0.039)\end{array}$ & $\begin{array}{c}0.019 \\
(0.038)\end{array}$ \\
\hline \multicolumn{7}{|l|}{ Model with covariates } \\
\hline $\begin{array}{l}\text { Problem behavior } \\
\text { (CR) }\end{array}$ & $\begin{array}{l}-0.006 \\
(0.027)\end{array}$ & $\begin{array}{l}-0.006 \\
(0.025)\end{array}$ & $\begin{array}{l}-0.002 \\
(0.031)\end{array}$ & $\begin{array}{l}-0.002 \\
(0.028)\end{array}$ & $\begin{array}{l}-0.006 \\
(0.027)\end{array}$ & $\begin{array}{l}-0.006 \\
(0.025)\end{array}$ \\
\hline $\begin{array}{l}\text { Normative beliefs about } \\
\text { aggression (CR) }\end{array}$ & $\begin{array}{l}-0.005 \\
(0.026)\end{array}$ & $\begin{array}{l}-0.005 \\
(0.024)\end{array}$ & $\begin{array}{l}-0.009 \\
(0.033)\end{array}$ & $\begin{array}{l}-0.009 \\
(0.030)\end{array}$ & $\begin{array}{l}-0.005 \\
(0.026)\end{array}$ & $\begin{array}{l}-0.005 \\
(0.025)\end{array}$ \\
\hline $\begin{array}{l}\text { Student afraid at school } \\
\text { (CR) }\end{array}$ & $\begin{array}{l}-0.067 * \\
(0.040)\end{array}$ & $\begin{array}{l}-0.067 * \\
(0.035)\end{array}$ & $\begin{array}{l}-0.047 \\
(0.045)\end{array}$ & $\begin{array}{l}-0.047 \\
(0.040)\end{array}$ & $\begin{array}{l}-0.067 * \\
(0.037)\end{array}$ & $\begin{array}{l}-0.067 * \\
(0.036)\end{array}$ \\
\hline $\begin{array}{l}\text { Altruistic behavior } \\
(\mathrm{PCR})\end{array}$ & $\begin{array}{l}-0.016 \\
(0.028)\end{array}$ & $\begin{array}{l}-0.016 \\
(0.024)\end{array}$ & $\begin{array}{l}-0.013 \\
(0.031)\end{array}$ & $\begin{array}{l}-0.013 \\
(0.027)\end{array}$ & $\begin{array}{l}-0.017 \\
(0.027)\end{array}$ & $\begin{array}{l}-0.017 \\
(0.026)\end{array}$ \\
\hline $\begin{array}{l}\text { Positive social behavior } \\
\text { (TR) }\end{array}$ & $\begin{array}{l}-0.011 \\
(0.045)\end{array}$ & $\begin{array}{l}-0.011 \\
(0.039)\end{array}$ & $\begin{array}{l}-0.015 \\
(0.045)\end{array}$ & $\begin{array}{l}-0.015 \\
(0.040)\end{array}$ & $\begin{array}{l}-0.010 \\
(0.043)\end{array}$ & $\begin{array}{l}-0.010 \\
(0.040)\end{array}$ \\
\hline $\begin{array}{l}\text { Problem behavior } \\
\text { (TR) }\end{array}$ & $\begin{array}{l}-0.009 \\
(0.025)\end{array}$ & $\begin{array}{l}-0.009 \\
(0.023)\end{array}$ & $\begin{array}{l}-0.011 \\
(0.026)\end{array}$ & $\begin{array}{l}-0.011 \\
(0.023)\end{array}$ & $\begin{array}{l}-0.009 \\
(0.025)\end{array}$ & $\begin{array}{l}-0.009 \\
(0.023)\end{array}$ \\
\hline
\end{tabular}

Abbreviations. $\mathrm{CR}=$ child report, $\mathrm{PCR}=$ primary caregiver report, $\mathrm{TR}=$ teacher report, $\mathrm{CRSE}=\mathrm{Cluster}-$ robust standard error estimator.

* Statistically significant at the 10 percent level, two-tailed test. 


\section{References}

Abadie A., Athey, S., Imbens, G., and Wooldridge, J. (2017), "When Should You Adjust Standard Errors for Clustering?" arxiv: 1710.02926[Math.ST]

Angrist, J. and Pischke, S. (2009), Mostly Harmless Econometrics, Princeton NJ: Princeton University Press.

Angrist, J. D. and Lavy, V. (2002), "The Effect of High School Matriculation Awards: Evidence from Randomized Trials," American Economic Review, 99, 1384-1414.

Aronow, P. M. and Middleton, J. A. (2013), “A Class of Unbiased Estimators of the Average Treatment Effect in Randomized Experiments," Journal of Causal Inference, 1, 135-154.

Aronow, P. M., Green, D. P. and Lee, D. K. K. (2014), "Sharp Bounds on the Variance in Randomized Experiments," Annals of Statistics," 42, 850-871.

Bell, R. and McCaffrey, D. (2002), "Bias Reduction in Standard Errors for Linear Regression with Multi-Stage Samples," Survey Methodology, 28, 169-181.

Bickel, B. J. and Freedman, D. A. (1984), "Asymptotic Normality and the Bootstrap in Stratified Sampling," The Annals of Statistics, 12(2), 470-482.

Bland, J. M. (2004), "Cluster Randomised Trials in the Medical Literature: Two Bibliometric Surveys," BMC Medical Research Methodology, 4, 21.

Bloniarz A., Liu H., Zhang C, Sekhon J. S., and Yu B. (2016), "Lasso Adjustments of Treatment Effect Estimates in Randomized Experiments," Proceedings of the National Academy of Sciences, 113: 7383-7390.

Cameron, A. C., and Miller, D. L. (2015), “A Practitioner's Guide to Cluster-Robust Inference," Journal of Human Resources, 50, 317-372.

Cameron, A. C., Gelbach, J. G., and Miller, D. L. (2008), "Bootstrap-Based Improvements for Inference with Clustered Errors," Review of Economics and Statistics, 90, 414-27.

Cochran, W. (1977), Sampling Techniques, New York: John Wiley and Sons.

Ding, P., Feller, A., \& Miratrix, L. (2018). Decomposing treatment effect variation. Journal of the American Statistical Association, 114(525), 304-317.

Donald, S. G., and Lang, K. L. (2007), "Inference with Difference-in-Differences and Other Panel Data," Review of Economics and Statistics, 89, 221-33.

Freedman, D. (2008), “On Regression Adjustments to Experimental Data," Advances in Applied Mathematics 40, 180-193.

Fuller, W. A. (1975), “Regression Analysis for Sample Survey,” Sankya, 37, Series C, 117-132.

Fuller, W. A. (2009), Sampling Statistics, Hoboken, NJ: Wiley. 
Green, D.P. and Vavrek, L. (2008), "Analysis of Cluster-Randomized Experiments: A Comparison of Alternative Estimation Approaches," Political Analysis, 16, 138-152.

Hansen, C. B. (2007), “Asymptotic Properties of a Robust Variance Matrix Estimator for Panel Data When T Is Large,” Journal of Econometrics, 141, 597-620.

Hansen, B. B., and Bowers, J. (2009), “Attributing Effects to a Cluster-Randomized Get-Outthe-Vote Campaign," Journal of the American Statistical Association, 104, 873-885.

Hedges, L. (2007), "Correcting a Significance Test for Clustering," Journal of Educational and Behavioral Statistics, 32, 151-179.

Holland, P. W. (1986), "Statistics and Causal Inference," Journal of the American Statistical Association, 81, 945-960.

Huber, P. J. (1967), “The Behavior of Maximum Likelihood Estimates Under Nonstandard Conditions," Procedures of the Fifth Berkeley Symposium on Math and Statistical Probability, 1, 221-233.

Imai, K., King, G. and Nall, C. (2009), "The Essential Role of Pair Matching in ClusterRandomized Experiments, with an Application to the Mexican Universal Health Insurance Evaluation," Statistical Science, 24, 29-53.

Imbens G. (2004), "Nonparametric Estimation of Average Treatment Effects Under Exogeneity: A Review," Review of Economics and Statistics, 86, 4-29.

Imbens, G. and Rubin, D. (2015), Causal inference for Statistics, Social, and Biomedical Sciences: An Introduction, Cambridge, UK: Cambridge University Press.

Kish, L. (1995), Survey Sampling, New York, NY: John Wiley and Sons.

Li, X. and Ding, P. (2017), "General Forms of Finite Population Central Limit Theorems with Applications to Causal inference," Journal of the American Statistical Association, 112, 1759-1769.

Liu, H. and Yang, Y. (2020), "Regression-Adjusted Average Treatment Effect Estimates in Stratified Randomized Experiments, Biometrika, asaa038, https://doi.org/10.1093/biomet/asaa038.

Liang, K. and Zeger, S. (1986), "Longitudinal Data Analysis Using Generalized Linear Models," Biometrika, 73, 13-22.

Lin, W. (2013), “Agnostic Notes on Regression Adjustments to Experimental Data:

Reexamining Freedman's Critique,” Annals of Applied Statistics, 7, 295-318.

Lohr, S. L. (2009), Sampling: Design and Analysis, Second Edition. Pacific Grove, CA: Duxbury Press.

Mackinnon, J. G. and White, H. (1985), "Some Heteroskedasticity-Consistent Covariance Matrix Estimators with Improved Finite Sample Properties," Journal of Econometrics 29, 305-25. 
Middleton, J. A. (2018), “A Unified Theory of Regression Adjustment for Design-based Inference," https://arxiv.org/abs/1803.06011.

Middleton, J. A. and Aronow, P. M. (2015), "Unbiased Estimation of the Average Treatment Effect in Cluster-Randomized Experiments," Statistics, Politics and Policy, 6, 39-75.

Miratrix, L.W., Sekhon, J. B. and Yu, B. (2013), "Adjusting Treatment Effect Estimates in Randomized Experiments," Journal of the Royal Statistical Society B, 75, 369-396.

Neyman, J. (1923), "On the Application of Probability Theory to Agricultural Experiments: Essay on Principles," Section 9, Translated in Statistical Science, 1990, 5, 465-472.

Pashley, N. E., and Miratrix, L. W. "Insights on variance estimation for blocked and matched pairs designs." Journal of Educational and Behavioral Statistics (2020): Online First.

Pustejovsky, J. E., and Tipton, E. (2018), "Small-Sample Methods for Cluster-Robust Variance Estimation and Hypothesis Testing in Fixed Effects Models," Journal of Business and Economic Statistics, 36, 672-683.

Rao, J. N. K., and Shao, J. (1999), "Modified Balanced Repeated Replication for Complex Survey Data," Biometrika, 86, 403-415.

Rubin, D. B. (1974), "Estimating Causal Effects of Treatments in Randomized and Nonrandomized Studies," Journal of Education Psychology, 66, 688-701.

Rubin, D. B. (1977), “Assignment to Treatment Group on the Basis of a Covariate," Journal of Education Statistics, 2, 1-26.

Rubin, D. B. (1986), "Which Ifs Have Causal Answers? Discussion of Holland's "Statistics and Causal inference," Journal of the American Statistical Association, 81, 961-962.

SACD Research Consortium (2010). "Efficacy of Schoolwide Programs to Promote Social and Character Development and Reduce Problem Behavior in Elementary School Children." Washington DC: Final Report: Institute for Education Sciences, U.S. Department of Education. https://ies.ed.gov/ncer/pubs/20112001/

Samii, C. and Aronow, P. M. (2012), "On Equivalencies Between Design-Based and RegressionBased Variance Estimators for Randomized Experiments," Statistics \& Probability Letters, 82, 365-370.

Schochet, P. Z. (2008), "Statistical Power for Random Assignment Evaluations of Education Programs," Journal of Educational and Behavioral Statistics, 33, 62-87.

Schochet, P. Z. (2010), "Is Regression Adjustment Supported by the Neyman Model for Causal inference?" Journal of Statistical Planning and inference, 140, 246-259.

Schochet, P. Z. (2013), "Estimators for Clustered Education RCTs Using the Neyman Model for Causal inference," Journal of Educational and Behavioral Statistics, 38, 219-238. 
Schochet, P. Z. (2016) Second Edition. Statistical Theory for the RCT-YES Software: DesignBased Causal inference for RCTs (NCEE 2015-4011), Washington, DC: National Center for Education Evaluation and Regional Assistance, Institute of Education Sciences, U.S. Department of Education. https://ies.id.gov/ncee/pubs/20154011/pdf/20154011.pdf.

Schochet P. Z. (2020a), "Analyzing Grouped Administrative Data for RCTs Using Design-Based Methods," Journal of Educational and Behavioral Statistics, 45: 32-57.

Schochet P. Z. (2020b), "A Lasso-OLS Hybrid Approach to Covariate Selection and Average Treatment Effect Estimation for Clustered RCTs Using Design-Based Methods.” Extracted from https://arxiv.org/abs/2005.02502, Under journal review.

Scott, A. and Wu, C. F. (1981), "On the Asymptotic Distribution of Ratio and Regression Estimators," Journal of the American Statistical Association, 112, 1759-1769.

Tibshirani, R. (1996), "Regression Shrinkage and Selection via the Lasso," Journal of the Royal Statistical Society. Series B, 58, 267-288.

Webb, M. D. (2013), "Reworking Wild Bootstrap Based Inference for Clustered Errors," Queens Economics Department Working Paper 1315.

White, H. (1980), "A Heteroskedasticity-Consistent Covariance Matrix Estimator and a Direct Test for Heteroskedasticity," Econometrica, 48, 817-838.

Wolter, K.M. (2007), Introduction to Variance Estimation, Second Edition. Springer Verlag.

Wooldridge, J. M. (2006), "Cluster Sample Methods in Applied Econometrics," Michigan State University, Working Paper.

Yang, L. and Tsiatis, A. (2001), "Efficiency Study of Estimators for a Treatment Effect in a Pretest-Posttest Trial," American Statistician, 55, 314-321. 


\section{Supplementary Materials for "Design-Based Ratio Estimators and Central Limit Theorems for Clustered, Blocked RCTs"}

\section{A Proofs for asymptotic results}

\section{A.1 Asymptotic properties of ratio estimators}

In this section, we develop two results that will help us prove asymptotic normality of our weighted least squares regression estimators. We derive these results generally, assuming arbitrary cluster level ${ }^{1}$ potential outcomes $C_{j}(t)$ for cluster $j$ with $t \in\{0,1\}$. These results could alternatively be obtained using a finite population delta method (see Pashley, 2019).

\section{A.1.1 Asymptotic normality of one ratio}

We first derive a result on the asymptotic distribution of a single ratio estimator, very similar to Theorem 1 in Scott and Wu (1981), but using conditions from Li and Ding (2017). To do this, we redefine the potential outcomes in a way that allows us to use known normality results for an estimator that is close to our ratio estimator. Then we use an application of Slutsky's theorem to get the normality result for our estimator of interest. Before the result, we must define some notation. Let us have arbitrary cluster level potential outcomes $C_{j}(t)$ for $t \in\{0,1\}$ and cluster weights $w_{j}$, with $T_{j}$ being the indicator of treatment assignment for cluster $j$. Following Li and Ding (2017), our finite population is within a sequence of finite populations where $m$ and $m^{t}$ go to $\infty$, which naturally holds if we grow the population with a fixed proportion of treated clusters, $p$. Further define

$$
\begin{aligned}
\bar{w} & =\frac{1}{m} \sum_{j=1}^{m} w_{j} \\
\bar{w}^{t} & =\frac{1}{m^{t}} \sum_{j: T_{j}=t} w_{j}, \\
\overline{\bar{C}}(t) & \left.=\frac{\frac{1}{m} \sum_{j=1}^{m} w_{j} C_{j}(t)}{\bar{w}} \quad \text { (the overall weighted mean of } C_{j}(t)\right), \\
\overline{\bar{c}}(t) & =\frac{\frac{1}{m^{t}} \sum_{j: T_{j}=t} w_{j} C_{j}(t)}{\bar{w}^{t}},
\end{aligned}
$$

\footnotetext{
${ }^{1}$ The same mathematical arguments are immediately extendable to the case where we have individuals rather than clusters.
} 
and

$$
\hat{\bar{C}}(t)=\overline{\bar{c}}(t) \bar{w}
$$

In particular, we are interested in the asymptotic distribution of the ratio estimator $\overline{\bar{c}}(t)$, unlike Scott and $\mathrm{Wu}$ (1981) who found the distribution for an estimator closer to the form $\hat{\bar{C}}(t)$. In our case, $\overline{\bar{c}}(t)$ is an estimator of $\overline{\bar{C}}(t)$. We will see this is a straightforward change.

Define the following finite population variance of the weights:

$$
S^{2}(w)=\frac{1}{m-1} \sum_{j=1}^{m}\left(w_{j}-\bar{w}\right)^{2}
$$

Let $D_{j}(t)$ by a scaled deviation of $C_{j}(t)$ from the overall weighted mean. That is,

$$
D_{j}(t)=\left(w_{j} C_{j}(t)-w_{j} \overline{\bar{C}}(t)\right) / \bar{w}
$$

Then the mean of the $D_{j}(t)^{\prime}$ 's, $\bar{D}(t)$, equals 0 as

$$
\sum_{j=1}^{m} w_{j} \overline{\bar{C}}(t)=m \overline{\bar{w}}(t)=\sum_{j=1}^{m} w_{j} C_{j}(t)
$$

and the variance expression for the $D_{j}(t)$, which is a weighted variance of the $C_{j}(t)$, is

$$
S_{D}^{2}(t)=\frac{1}{m-1} \sum_{j=1}^{m} D_{j}^{2}(t)=\frac{1}{m-1} \sum_{j=1}^{m} \frac{w_{j}^{2}}{\bar{w}^{2}}\left(C_{j}(t)-\overline{\bar{C}}(t)\right)^{2}
$$

Lemma A.1.1. Assume we have the following conditions:

(a) Defining $g(t)=\max _{1 \leq j \leq m}\left(D_{j}(t)\right)^{2}$

$$
\frac{1}{\min \left(m^{1}, m-m^{1}\right)} \frac{g(t)}{S_{D}^{2}(t)} \rightarrow 0, \quad \text { as } m \rightarrow \infty .
$$

(b) For $t \in\{1,0\}$,

$$
\left(1-f^{t}\right) \frac{S^{2}(w)}{m^{t} \bar{w}^{2}} \rightarrow 0 \text { as } m \rightarrow \infty
$$

Under these conditions,

$$
\frac{\overline{\bar{c}}(t)-\overline{\bar{C}}(t)}{\sqrt{\frac{1-f^{t}}{m^{t}}} S_{D}(t) / \bar{w}} \stackrel{d}{\rightarrow} N(0,1)
$$


Proof. The proof of this result closely follows the proof in Scott and Wu (1981). We are interested in the asymptotic distribution of the ratio estimator $\overline{\bar{c}}(t)$, but to find this we will first find the distribution of $\hat{\bar{C}}(t)$.

Let $\bar{d}(t)=\sum_{j: T_{j}=t} D_{j}(t) / m^{t}$. Then

$$
\bar{d}(t)=(\overline{\bar{c}}(t)-\overline{\bar{C}}(t)) \frac{\bar{w}^{t}}{\bar{w}} .
$$

Assuming our first condition given by Equation S1, by Theorem 1 of Li and Ding (2017),

$$
\frac{\bar{d}(t)}{\sqrt{\frac{1-f^{t}}{m^{t}}} S_{D}(t)} \stackrel{d}{\rightarrow} N(0,1) .
$$

Further, from Theorem B of Scott and Wu (1981), given our second condition given by Equation S2,

$$
\bar{w}^{t} / \bar{w}-1 \stackrel{p}{\rightarrow} 0 \text { as } m \rightarrow \infty .
$$

Thus, $\bar{w}^{t} / \bar{w} \stackrel{p}{\rightarrow} 1$. Note the condition on the weights would cause issues if the average weight were to go to 0 as the sample size increased, but, as discussed in Section A.2.3, this is easy to avoid by using unnormalized weights. This in turn means by Slutsky's Theorem, recalling that $\overline{\bar{c}}(t)-\overline{\bar{C}}(t)=\bar{d}(t) \bar{w} / \bar{w}^{t}$, we have our result in Equation S3.

\section{A.1.2 Asymptotic normality of difference of ratios (Main result)}

We will now show the asymptotic normality of a difference of ratios. In particular, we are interested in the asymptotic distribution of $\overline{\bar{c}}(1)-\overline{\bar{c}}(0)$, using notation from Section A.1.1. We will again define new potential outcomes that are easier to work with than the direct ratio. First we define some notation. Construct new potential outcomes $D_{j}(t)=\left(w_{j} C_{j}(t)-\right.$ $\left.w_{j} \overline{\bar{C}}(t)\right) / \bar{w}$ with notation defined in Section A.1.1, so $\bar{D}(1)=0, \bar{D}(0)=0$ and

$$
\bar{d}(t)=\frac{1}{m^{t}} \sum_{j: T_{j}=t} D_{j}(t) .
$$

Further define an intermediate estimator $\hat{D}=\bar{d}(1)-\bar{d}(0)$ and

$$
S_{D}^{2}(1,0)=\frac{1}{m-1} \sum_{j=1}^{m} D_{j}(1) D_{j}(0) .
$$

Recall for $t \in\{0,1\}$

$$
S_{D}^{2}(t)=\frac{1}{m-1} \sum_{j=1}^{m} D_{j}^{2}(t)
$$

and further define

$$
S^{2}(D)=\frac{1}{m-1} \sum_{j=1}^{m}\left(D_{j}(1)-D_{j}(0)\right)^{2} .
$$

We will use these notations to get the final result. 
Lemma A.1.2. Let us have the following conditions ${ }^{2}$ :

(a) Let $g(t)=\max _{1 \leq j \leq m}\left(D_{j}(t)\right)^{2}$ and as $m \rightarrow \infty$

$$
\max _{t \in\{0,1\}} \frac{1}{\left(m^{t}\right)^{2}} \frac{g(t)}{\operatorname{Var}(\hat{D})} \rightarrow 0 .
$$

(b) $m^{t} / m$ has a limiting value in $(0,1)$ and $S_{D}^{2}(t)$ and $S_{D}^{2}(1,0)$ have limiting values.

(c) For $t \in\{1,0\}$,

$$
\left(1-f^{t}\right) \frac{S^{2}(w)}{m^{t} \bar{w}^{2}} \rightarrow 0 \text { as } m \rightarrow \infty
$$

Then we have that

$$
\frac{(\overline{\bar{c}}(1)-\overline{\bar{c}}(0))-(\overline{\bar{C}}(1)-\overline{\bar{C}}(0))}{\sqrt{\operatorname{Var}(\hat{D})}} \stackrel{d}{\rightarrow} N(0,1)
$$

where

$$
\operatorname{Var}(\hat{D})=\frac{S_{D}^{2}(1)}{m^{1}}+\frac{S_{D}^{2}(0)}{m^{0}}-\frac{S^{2}(D)}{m} .
$$

Proof. First we derive a normality result for $\hat{D}$. If we randomly select $p m$ clusters to assign to treatment, then from Theorem 3 in Li and Ding (2017), $\hat{D}$ has mean 0 and variance

$$
\operatorname{Var}(\hat{D})=\frac{S_{D}^{2}(1)}{m^{1}}+\frac{S_{D}^{2}(0)}{m^{0}}-\frac{S^{2}(D)}{m} .
$$

If we have the condition of Equation S4, then under Theorem 4 of Li and Ding (2017),

$$
\frac{\hat{D}}{\sqrt{\operatorname{Var}(\hat{D})}} \stackrel{d}{\rightarrow} \mathrm{N}(0,1) \text {. }
$$

Now note that

$$
\begin{aligned}
\frac{\hat{D}}{\sqrt{\operatorname{Var}(\hat{D})}} & =\frac{\bar{d}(1)-\bar{d}(0)}{\sqrt{\operatorname{Var}(\hat{D})}} \\
& =\frac{\frac{1}{m^{1}} \sum_{j=1}^{m} T_{j}\left(w_{j} C_{j}(1)-w_{j} \overline{\bar{C}}(1)\right) / \bar{w}-\frac{1}{m^{0}} \sum_{j=1}^{m}\left(1-T_{j}\right)\left(w_{j} C_{j}(0)-w_{j} \overline{\bar{C}}(0)\right) / \bar{w}}{\sqrt{\operatorname{Var}(\hat{D})}} \\
& =\frac{\frac{\bar{w}^{1}}{\bar{w}}(\overline{\bar{c}}(1)-\overline{\bar{C}}(1))-\frac{\bar{w}^{0}}{\bar{w}}(\overline{\bar{c}}(0)-\overline{\bar{C}}(0))}{\sqrt{\operatorname{Var}(\hat{D})}} .
\end{aligned}
$$

\footnotetext{
${ }^{2}$ It is interesting to note that a delta method approach would rely on slightly different conditions. In particular, the delta method does not explicitly require limiting values on asymptotic variances, as given in the second condition, but it does require a convergence in probability result that is satisfied by those limiting values for the asymptotic variances. Comparing this derivation to proofs via the delta method is left for future work.
} 
We can now get back to our estimator of interest, $\overline{\bar{c}}(1)-\overline{\bar{c}}(0)$ :

$$
\begin{aligned}
& \frac{(\overline{\bar{c}}(1)-\overline{\bar{C}}(1))-(\overline{\bar{c}}(0)-\overline{\bar{C}}(0))}{\sqrt{\operatorname{Var}(\hat{D})}} \\
& =\frac{\left(1-\frac{\bar{w}^{1}}{\bar{w}}\right)(\overline{\bar{c}}(1)-\overline{\bar{C}}(1))-\left(1-\frac{\bar{w}^{0}}{\bar{w}}\right)(\overline{\bar{c}}(0)-\overline{\bar{C}}(0))}{\sqrt{\operatorname{Var}(\hat{D})}} \\
& +\frac{\frac{\bar{w}^{1}}{\bar{w}}(\overline{\bar{c}}(1)-\overline{\bar{C}}(1))-\frac{\bar{w}^{0}}{\bar{w}}(\overline{\bar{c}}(0)-\overline{\bar{C}}(0))}{\sqrt{\operatorname{Var}(\hat{D})}} \\
& =\left(1-\frac{\bar{w}^{1}}{\bar{w}}\right) \frac{(\overline{\bar{c}}(1)-\overline{\bar{C}}(1))}{\sqrt{\operatorname{Var}(\hat{D})}}-\left(1-\frac{\bar{w}^{0}}{\bar{w}}\right) \frac{(\overline{\bar{c}}(0)-\overline{\bar{C}}(0))}{\sqrt{\operatorname{Var}(\hat{D})}} \\
& +\frac{\hat{D}}{\sqrt{\operatorname{Var}(\hat{D})}}
\end{aligned}
$$

We know the asymptotic distribution of the third term, so we need to show that the first two terms vanish. As stated in Lemma A.1.1, if we have the conditions given by Equation S4 and Equation S5, then

$$
\frac{\overline{\bar{c}}(t)-\overline{\bar{C}}(t)}{\sqrt{\frac{1-f^{t}}{m^{t}}} S_{D}(t)} \stackrel{d}{\rightarrow} N(0,1) .
$$

Recall from Section A.1.1 that the fourth condition gives us

$$
\frac{\bar{w}^{t}}{\bar{w}} \stackrel{p}{\rightarrow} 1
$$

To use these notes, we need to rewrite out previous result:

$$
\begin{aligned}
\frac{(\overline{\bar{c}}(1)-\overline{\bar{C}}(1))-(\overline{\bar{c}}(0)-\overline{\bar{C}}(0))}{\sqrt{\operatorname{Var}(\hat{D})}}=\left(1-\frac{\bar{w}^{1}}{\bar{w}}\right) \frac{\sqrt{\frac{1}{m^{1}}-\frac{1}{m}} S_{D}(1)}{\sqrt{\operatorname{Var}(\hat{D})}} \frac{(\overline{\bar{c}}(1)-\overline{\bar{C}}(1))}{\sqrt{\frac{1}{m^{1}}-\frac{1}{m}} S_{D}(1)} \\
\\
-\left(1-\frac{\bar{w}^{0}}{\bar{w}}\right) \frac{\sqrt{\frac{1}{m^{0}}-\frac{1}{m}} S_{D}(0)}{\sqrt{\operatorname{Var}(\hat{D})}} \frac{(\overline{\bar{c}}(0)-\overline{\bar{C}}(0))}{\sqrt{\frac{1}{m^{0}}-\frac{1}{m}} S_{D}(0)} \\
+\frac{\hat{D}}{\sqrt{\operatorname{Var}(\hat{D})}} .
\end{aligned}
$$

The second condition puts limiting values on our asymptotic variances. That is, the second condition (see Theorems 3 and 5 of Li and Ding (2017)), gives us that for $t \in\{0,1\}$, 
$m\left(\frac{1-f^{t}}{m^{t}}\right) S_{D}^{2}(t)$ has some limiting value $\sigma_{D}^{2}(t)$ and $m \operatorname{Var}(\hat{D})$ has some limiting value $V$. This allows us to use Slutsky's theorem to get

$$
\left(1-\frac{\bar{w}^{1}}{\bar{w}}\right) \frac{\sqrt{\frac{1}{m^{1}}-\frac{1}{m}} S_{D}(t) / \bar{w}}{\sqrt{\operatorname{Var}(\hat{D})}} \frac{(\overline{\bar{c}}(t)-\overline{\bar{C}}(t))}{\sqrt{\frac{1-f^{t}}{m^{t}}} S_{D}(t)} \stackrel{p}{\rightarrow} 0 .
$$

To break this step down more, note that the first term goes to 0 in probability, the second goes to a constant by the third condition, and the final term is asymptotically normal.

Finally, Slutsky's theorem gives the result in Equation S6.

\section{A.2 Theorem 1 without covariates}

In this section, we explore the impact estimator of a weighted least squares regression without covariate adjustment. We prove Theorem 1 under this special case and also find the bias of our estimator. We start by deriving the form of the estimator, then finding bias and consistency results. In particular, the estimator has finite sample bias but is consistent. We then show the asymptotic normality result given in Theorem 1 . Without covariates, the interactions in our regression imply that block means and effects are estimated independently, and so we can focus on the estimator for a single block, $b$. In other words, for now we can act as if there is only one block.

\section{A.2.1 The estimator}

We assume that we have one block, arbitrarily block $b$, with $m_{b}$ clusters. We assign $m_{b}^{1}=$ $p_{b} m_{b}$ clusters to treatment and the rest of the $m_{b}^{0}$ clusters to control. Define shorthand $f_{b}^{t}=m_{b}^{t} / m_{b}$. When we have multiple blocks, we have block indicators $S_{i j b}$ and $S_{j b}$ which equals 1 if unit $i$ or cluster $j$ belong to block $b$, and 0 otherwise. Each unit $i$, of $n_{j b}$ units, in cluster $j$ of block $b$ has associated weight $w_{i j b}$ and we have $w_{j b}=\sum w_{i j b}$. Denote $w_{b}^{0}=\sum_{j=1}^{m_{b}}\left(1-T_{j b}\right) w_{j b}, w_{b}^{1}=\sum_{j=1}^{m_{b}} T_{j b} w_{j b}$, and $w_{b}=\sum_{j=1}^{m_{b}} w_{j b}$. Each unit $i$ in cluster $j$ has potential outcomes $Y_{i j b}(1)$ under treatment and $Y_{i j b}(0)$ under control, with treatment assigned at the cluster level. $y_{i j b}$ is the observed outcome for unit $i$ in cluster $j$ of block $b$ and $\bar{y}_{j b}$ is the observed weighted average outcome for all units in cluster $j$ of block $b$. We have by definition that $y_{i j b}=T_{j b} Y_{i j b}(1)+\left(1-T_{j b}\right) Y_{i j b}(0)$ and for $t \in\{0,1\}$,

$$
\bar{Y}_{j b}(t)=\frac{1}{w_{j b}} \sum_{i: T_{j b}=t} w_{i j b} Y_{i j b}(t)
$$

and $\bar{y}_{j b}=T_{j b} \bar{Y}_{j b}(1)+\left(1-T_{j b}\right) \bar{Y}_{j b}(0)$. We also define for $t \in\{0,1\}$,

$$
\overline{\bar{y}}_{b}(t)=\frac{1}{\sum_{j: T_{j b}=t} w_{j b}} \sum_{j: T_{j b}=t} w_{j b} \bar{y}_{j b} .
$$

We have finite population parameters

$$
\overline{\bar{Y}}_{b}(t)=\frac{1}{\sum_{j=1}^{m_{b}} w_{j b}} \sum_{j=1}^{m_{b}} w_{j b} \bar{Y}_{j b}(t)
$$


Throughout, we will treat $\beta_{1, b}$ as the finite population parameter of the weighted average of cluster impacts,

$$
\beta_{1, b}=\frac{\sum_{j=1}^{m_{b}} w_{j b}\left(\bar{Y}_{j b}(1)-\bar{Y}_{j b}(0)\right)}{\sum_{j=1}^{m_{b}} w_{j b}}=\overline{\bar{Y}}_{b}(1)-\overline{\bar{Y}}_{b}(0) .
$$

We start by finding the exact form of the regression estimator $\hat{\beta}_{1}$. For our regression, we have $\boldsymbol{z}_{i j b}=\left(S_{i j b} \tilde{T}_{j b}, S_{i j b}\right)=\left(\tilde{T}_{j b}, 1\right)$ because we only have one block. Here we define

$$
p_{b}^{*}=\frac{1}{w_{b}} \sum_{j=1}^{m_{b}} T_{j b} w_{j b}=\frac{w_{b}^{1}}{w_{b}}
$$

and

$$
\tilde{T}_{j b}=T_{j b}-p_{b}^{*},
$$

so that

$$
\sum_{j=1}^{m_{b}} w_{j_{b}} \tilde{T}_{j b}=\sum_{j=1}^{m_{b}} w_{j_{b}}\left(T_{j b}-p_{b}^{*}\right)=0 .
$$

The estimated parameter vector from weighted least squares regressing the individual $y_{i j b}$ on an intercept and centered treatment indicator $\tilde{T}_{j b}$ with weights $w_{i j b}$ is

$$
\left(\begin{array}{l}
\hat{\beta}_{1, b} \\
\hat{\beta}_{0, b}
\end{array}\right)=\left[\left(\sum_{j=1}^{m_{b}} \sum_{i=1}^{n_{j b}} w_{i j b} \boldsymbol{z}_{i j b}^{\prime} \boldsymbol{z}_{i j b}\right)^{-1} \sum_{j=1}^{m_{b}} \sum_{i=1}^{n_{j b}} w_{i j b} \boldsymbol{z}_{i j b}^{\prime} y_{i j b}\right] .
$$

Result: $\hat{\beta}_{1, b}=\overline{\bar{y}}_{b}(1)-\overline{\bar{y}}_{b}(0)$.

We have by simple algebra simplifications and matrix inversion,

$$
\begin{aligned}
\left(\begin{array}{c}
\hat{\beta}_{1, b} \\
\hat{\beta}_{0, b}
\end{array}\right) & =\left(\begin{array}{cc}
\sum_{j=1}^{m_{b}} w_{j b} \tilde{T}_{j b}^{2} & \sum_{j=1}^{m_{b}} w_{j b} \tilde{T}_{j b} \\
\sum_{j=1}^{m_{b}} w_{j b} \tilde{T}_{j b} & \sum_{j=1}^{m_{b}} w_{j b}
\end{array}\right)^{-1}\left(\begin{array}{c}
\sum_{j=1}^{m_{b}} w_{j b} \tilde{T}_{j b} \bar{y}_{j b} \\
\sum_{j=1}^{m_{b}} w_{j b} \bar{y}_{j b}
\end{array}\right) \\
& =\left(\begin{array}{cc}
\sum_{j=1}^{m_{b}} w_{j b} \tilde{T}_{j b}^{2} & 0 \\
0 & w_{b}
\end{array}\right)^{-1}\left(\begin{array}{c}
\sum_{j b}^{m_{b}} w_{j b} \tilde{T}_{j b} \bar{y}_{j b} \\
w_{b}^{1} \overline{\bar{y}}_{b}(1)+w_{b}^{0} \overline{\bar{y}}_{b}(0)
\end{array}\right) \\
& =\left(\begin{array}{cc}
\frac{1}{\sum_{j=1}^{m_{b}} w_{j b} \tilde{T}_{j b}^{2}} & 0 \\
0 & \frac{1}{w_{b}}
\end{array}\right)\left(\begin{array}{c}
\sum_{j=1}^{m_{b}} w_{j b} \tilde{T}_{j b} \bar{y}_{j b} \\
w_{b}^{1} \overline{\bar{y}}_{b}(1)+w_{b}^{0} \overline{\bar{y}}_{b}(0)
\end{array}\right)
\end{aligned}
$$

To simplify this final form, note a few useful algebra results:

$$
\begin{aligned}
\sum_{j=1}^{m_{b}} w_{j b} \tilde{T}_{j b}^{2} & =\sum_{j=1}^{m_{b}} w_{j b}\left(T_{j b}^{2}-2 T_{j b} p_{b}^{*}+\left(p_{b}^{*}\right)^{2}\right) \\
& =w_{b}^{1}-\frac{\left(w_{b}^{1}\right)^{2}}{w_{b}} \\
& =\frac{w_{b}^{1} w_{b}^{0}}{w_{b}}
\end{aligned}
$$


and

$$
\begin{aligned}
\sum_{j=1}^{m_{b}} w_{j b} \tilde{T}_{j b} \bar{y}_{j b} & =\sum_{j=1}^{m_{b}} w_{j b}\left(T_{j b}-p_{b}^{*}\right) \bar{y}_{j b} \\
& =w_{b}^{1} \overline{\bar{y}}_{b}(1)-\frac{w_{b}^{1}}{w_{b}}\left(w_{b}^{1} \overline{\bar{y}}_{b}(1)+w_{b}^{0} \overline{\bar{y}}_{b}(0)\right) \\
& =\frac{w_{b}^{1} w_{b}^{0}}{w_{b}}\left(\overline{\bar{y}}_{b}(1)-\overline{\bar{y}}_{b}(0)\right) .
\end{aligned}
$$

We then have the following for our regression estimator for the treatment impact (and control mean):

$$
\begin{aligned}
\left(\begin{array}{c}
\hat{\beta}_{1, b} \\
\hat{\beta}_{0, b}
\end{array}\right) & =\left(\begin{array}{cc}
\frac{w_{b}}{w_{b}^{1} w_{b}^{0}} & 0 \\
0 & \frac{1}{w_{b}}
\end{array}\right)\left(\begin{array}{c}
\frac{w_{b}^{1} w_{b}^{0}}{w_{b}}\left(\overline{\bar{y}}_{b}(1)-\overline{\bar{y}}_{b}(0)\right) \\
w_{b}^{1} \overline{\bar{y}}_{b}(1)+w_{b}^{0} \overline{\bar{y}}_{b}(0)
\end{array}\right) \\
& =\left(\begin{array}{c}
\overline{\bar{y}}_{b}(1)-\overline{\bar{y}}_{b}(0) \\
\frac{w_{b}^{1}}{w_{b}} \overline{\bar{y}}_{b}(1)+\frac{w_{b}^{0}}{w_{b}} \overline{\bar{y}}_{b}(0)
\end{array}\right) .
\end{aligned}
$$

\section{A.2.2 Finite sample bias}

Here we explore the finite sample bias of the regression estimator. Using Theorem 1 in Middleton (2008) or Equation 7 in Middleton and Aronow (2015), originally from Hartley and Ross (1954), the bias for a ratio estimator is

$$
E\left[\frac{u}{v}\right]=\frac{1}{E[v]}\left[E[u]-\operatorname{Cov}\left(\frac{u}{v}, v\right)\right]
$$

assuming that $v>0$.

We then have, assuming that $w^{1}>0$ and $w^{0}>0$,

$$
\begin{aligned}
E\left[\overline{\bar{y}}_{b}(1)\right] & =E\left[\frac{\sum_{j=1}^{m_{b}} T_{j b} w_{j b} \bar{y}_{b j}}{\sum_{j=1}^{m_{b}} w_{j b} T_{j b}}\right] \\
& =\frac{1}{\frac{p_{b}}{m_{b}^{1}} \sum_{j=1}^{m_{b}} w_{j b}}\left[\frac{p_{b}}{m_{b}^{1}} \sum_{j=1}^{m_{b}} w_{j b} \bar{Y}_{j b}(1)-\operatorname{Cov}\left(\frac{\sum_{j=1}^{m_{b}} T_{j b} w_{j b} \bar{y}_{j b}}{\sum_{j=1}^{m_{b}} w_{j b} T_{j b}}, \frac{1}{m_{b}^{1}} \sum_{j=1}^{m_{b}} T_{j b} w_{j b}\right)\right] \\
& =\overline{\bar{Y}}_{b}(1)-\frac{m_{b}}{w_{b}} \operatorname{Cov}\left(\overline{\bar{y}}_{b}(1), \frac{w_{b}^{1}}{m_{b}^{1}}\right) .
\end{aligned}
$$

It is then straightforward to find the bias of our estimator as follows:

$$
E\left[\hat{\beta}_{1, b}\right]-\beta_{1, b}=-\frac{m_{b}}{w_{b}} \operatorname{Cov}\left(\overline{\bar{y}}_{b}(1), \frac{w_{b}^{1}}{m_{b}^{1}}\right)+\frac{m_{b}}{w_{b}} \operatorname{Cov}\left(\overline{\bar{y}}_{b}(0), \frac{w_{b}^{0}}{m_{b}^{0}}\right) .
$$

This means, as pointed out by Middleton and Aronow (2015), that the bias will depend on (1) the covariance between cluster size (as captured by the $w_{j b}$ ) and outcomes and (2) the variability of cluster sizes. 


\section{A.2.3 Consistency}

Here we give conditions for consistency of the regression estimator. It is useful to assume limiting values on the finite population parameters. This can be handled in a few ways. Following Middleton and Aronow (2015), we may envision a sequence of $h$ finite populations such that as $h \rightarrow \infty$, the finite population increases by copying the original $m_{b}$ clusters in block $b h$ times and then randomization occurs independently within each copy with probability $p_{b}$. This keeps our estimands and parameters constant as the population grows.

A bit more generally, we may have limiting values for the finite population parameters. For a given finite population with $m$ clusters, define the following quantities:

$$
\begin{aligned}
& {\overline{w Y(1)_{b}}}=\frac{1}{m_{b}} \sum_{j=1}^{m_{b}} w_{j b} \bar{Y}_{j b}(1), \\
& \overline{w Y(0)_{b}}=\frac{1}{m_{b}} \sum_{j=1}^{m_{b}} w_{j b} \bar{Y}_{j b}(0),
\end{aligned}
$$

and

$$
\bar{w}_{b}=\frac{1}{m_{b}} \sum_{j=1}^{m_{b}} w_{j b} .
$$

Given these definitions, our $\beta_{1, b}$ is then

$$
\beta_{1, b}=\frac{{\overline{w Y(1)_{b}}}_{b}}{\bar{w}_{b}}-\frac{\overline{w Y(0)}_{b}}{\bar{w}_{b}} .
$$

Assume limiting values $\mu_{b}^{*}(1), \mu_{b}^{*}(0)$, and $\omega_{b}$ such that as $m_{b} \rightarrow \infty$,

$$
\begin{aligned}
& \overline{w Y(1)_{b}} \stackrel{p}{\rightarrow} \mu_{b}^{*}(1), \\
& \overline{w Y(0)_{b}} \stackrel{p}{\rightarrow} \mu_{b}^{*}(0),
\end{aligned}
$$

and

$$
\bar{w}_{b} \stackrel{p}{\rightarrow} \omega_{b}>0
$$

If we were normalizing the weights across the whole population, it could be the case that $\omega_{b} \rightarrow 0$; we eliminate this issue by using unnormalized weights (which don't change the estimand or estimator because we use weights in the numerator and denominator). This gives a limiting value for $\beta_{1, b}$ of $\beta_{1, b}^{*}=\left(\mu_{b}^{*}(1)-\mu_{b}^{*}(0)\right) / \omega_{b}$.

We next show that $\hat{\beta}_{1, b} \stackrel{p}{\rightarrow} \beta_{1, b}^{*}$. Denote

$$
\begin{aligned}
& S_{w, b}^{2}(1)=\frac{1}{m_{b}-1} \sum_{j=1}^{m_{b}}\left(w_{j b} \bar{Y}_{j b}(1)-\overline{w Y(1)_{b}}\right)^{2} \\
& S_{w, b}^{2}(0)=\frac{1}{m_{b}-1} \sum_{j=1}^{m_{b}}\left(w_{j} \bar{Y}_{j b}(0)-\overline{w Y(0)_{b}}\right)^{2}
\end{aligned}
$$


and

$$
S^{2}\left(w_{b}\right)=\frac{1}{m_{b}-1} \sum_{j=1}^{m_{b}}\left(w_{j b}-\bar{w}_{b}\right)^{2} .
$$

Assume that $m_{b}^{1} / m_{b} \rightarrow p_{b} \in(0,1)$. Using Theorem B from Scott and Wu (1981), under simple random sampling of clusters into treatment, if our weighted variances do not go to infinity as we increase sample size, specifically if as $m_{b} \rightarrow \infty$,

$$
\begin{aligned}
& S_{w, b}^{2}(1) / m_{b} \rightarrow 0, \\
& S_{w, b}^{2}(0) / m_{b} \rightarrow 0,
\end{aligned}
$$

and

$$
S^{2}\left(w_{b}\right) / m_{b} \rightarrow 0
$$

then

$$
\begin{gathered}
\frac{1}{m_{b}^{1}} \sum_{j=1}^{m_{b}} w_{j b} T_{j b} \bar{Y}_{j b}(1)-\overline{w Y(1)_{b}} \stackrel{p}{\rightarrow} 0, \\
\frac{1}{m_{b}^{0}} \sum_{j=1}^{m_{b}} w_{j b}\left(1-T_{j b}\right) \bar{Y}_{j b}(0)-\overline{w Y(0)_{b}} \stackrel{p}{\rightarrow} 0, \\
\frac{1}{m_{b}^{1}} \sum_{j=1}^{m_{b}} w_{j b} T_{j b}-\bar{w}_{b} \stackrel{p}{\rightarrow} 0,
\end{gathered}
$$

and

$$
\frac{1}{m_{b}^{0}} \sum_{j=1}^{m_{b}} w_{j b}\left(1-T_{j b}\right)-\bar{w}_{b} \stackrel{p}{\rightarrow} 0
$$

as $m_{b} \rightarrow \infty$.

We next need a small convergence lemma:

Lemma A.2.1. If $A_{m}-B_{m} \stackrel{p}{\rightarrow} 0$ and $B_{m} \stackrel{p}{\rightarrow} k$, with $k$ a constant, as $m \rightarrow \infty$ then $A_{m} \stackrel{p}{\rightarrow} k$ as $m \rightarrow \infty$.

Proof.

$$
A_{m}-k=A_{m}-B_{m}+B_{m}-k \stackrel{p}{\rightarrow} 0
$$

Using Lemma A.2.1, we have

$$
\frac{1}{m_{b}^{t}} \sum_{j: T_{j b}=t} w_{j b} \bar{Y}_{j b}(t)=\frac{w_{b}^{t}}{m_{b}^{t}} \overline{\bar{y}}_{b}(t) \stackrel{p}{\rightarrow} \mu_{b}^{*}(t)
$$

and

$$
\frac{1}{m_{b}^{t}} \sum_{j: T_{j b}=t} w_{j b}=\frac{w_{b}^{t}}{m_{b}^{t}} \stackrel{p}{\rightarrow} \omega_{b}
$$


Then by Slutsky's theorem,

$$
\hat{\beta}_{1, b}=\overline{\bar{y}}_{b}(1)-\overline{\bar{y}}_{b}(0) \stackrel{p}{\rightarrow} \frac{\mu_{b}^{*}(1)}{\omega_{b}}-\frac{\mu_{b}^{*}(0)}{\omega_{b}}=\beta_{1, b}^{*} .
$$

Hence in this setting our estimator is consistent.

\section{A.2.4 Asymptotic normality of one ratio}

We are interested in the asymptotic behavior of, for $t \in\{0,1\}$,

$$
\overline{\bar{y}}_{b}(t)=\frac{1}{\sum_{j=1}^{m_{b}} w_{j b} T_{j b}} \sum_{j=1}^{m_{b}} T_{j b} w_{j b} \bar{Y}_{j b}(t) .
$$

We use the notation from Section A.1.1 but now add subscripts $b$ to indicate that we are referring to block $b$. For $t \in\{1,0\}$, let $C_{j b}(t)=\bar{Y}_{j b}(t)$, so that $\overline{\bar{c}}_{b}(t)=\overline{\bar{y}}_{b}(t)$ and $\overline{\bar{C}}_{b}(t)=\overline{\bar{Y}}_{b}(t)$.

Corollary A.2.2. Under the conditions of Lemma A.1.1, we have

$$
\begin{aligned}
\frac{\overline{\bar{c}}_{b}(t)-\overline{\bar{C}}_{b}(t)}{\sqrt{\frac{1-f_{b}^{t}}{m_{b}^{t}}} S_{R, b}(t) / \bar{w}} & =\frac{\left(\overline{\bar{y}}_{b}(t)-\overline{\bar{Y}}_{b}(t)\right)}{\sqrt{\frac{1-f_{b}^{t}}{m_{b}^{t}}} \sqrt{\frac{1}{m_{b}-1} \sum_{j=1}^{m_{b}}\left(R_{j b}(t) / \bar{w}_{b}\right)^{2}}} \\
& \stackrel{d}{\rightarrow} N(0,1) .
\end{aligned}
$$

The term in the denominator expands as follows:

$$
\begin{aligned}
\frac{1}{m_{b}-1} \sum_{j=1}^{m_{b}}\left(R_{j b}(t) / \bar{w}_{b}\right)^{2} & =\frac{1}{m_{b}-1} \sum_{j=1}^{m_{b}}\left(\left(w_{j b} C_{j b}(t)-w_{j b} \overline{\bar{C}}_{b}(t)\right) / \bar{w}_{b}\right)^{2} \\
& =\frac{1}{\left(m_{b}-1\right)} \sum_{j=1}^{m_{b}} \frac{w_{j b}^{2}}{\bar{w}_{b}^{2}}\left(\bar{Y}_{j b}(t)-\overline{\bar{Y}}_{b}(t)\right)^{2} .
\end{aligned}
$$

\section{A.2.5 Asymptotic normality}

We are interested in the ratio difference estimator, $\overline{\bar{y}}_{b}(1)-\overline{\bar{y}}_{b}(0)$.

Let $D_{j b}(t)=\left(w_{j b} C_{j b}(t)-w_{j b} \overline{\bar{C}}_{b}(t)\right) / \bar{w}_{b}=w_{j b}\left(\bar{Y}_{j b}(t)-\overline{\bar{Y}}_{b}(t)\right) / \bar{w}_{b}$, with $C_{j b}(t)$ defined as in Section A.2.4. Note that this means that we still have $\overline{\bar{c}}_{b}(t)=\overline{\bar{y}}_{b}(t)$ and $\overline{\bar{C}}_{b}(t)=\overline{\bar{Y}}_{b}(t)$. Now we have reformulated our complicated difference in ratio estimators into a form that's easier to work with.

Corollary A.2.3. Under the conditions of Lemma A.1.2, we have

$$
\begin{aligned}
\frac{\left(\overline{\bar{c}}_{b}(1)-\overline{\bar{C}}_{b}(1)\right)-\left(\overline{\bar{c}}_{b}(0)-\overline{\bar{C}}_{b}(0)\right)}{\sqrt{\operatorname{Var}\left(\hat{D}_{b}\right)}} & =\frac{\left(\overline{\bar{y}}_{b}(1)-\overline{\bar{Y}}_{b}(1)\right)-\left(\overline{\bar{y}}_{b}(0)-\overline{\bar{Y}}_{b}(0)\right)}{\sqrt{\operatorname{Var}\left(\hat{D}_{b}\right)}} \\
& \stackrel{d}{\rightarrow} N(0,1) .
\end{aligned}
$$


The denominator simplifies as follows:

$$
\begin{aligned}
& \operatorname{Var}\left(\hat{D}_{b}\right)= \frac{S_{D, b}^{2}(1)}{m_{b}^{1}}+\frac{S_{D, b}^{2}(0)}{m_{b}^{0}}-\frac{S^{2}\left(D_{b}\right)}{m_{b}} \\
&= \frac{\frac{1}{m_{b}-1} \sum_{j=1}^{m_{b}} D_{j b}^{2}(1)}{m_{b}^{1}}+\frac{\frac{1}{m_{b}-1} \sum_{j=1}^{m_{b}} D_{j b}^{2}(0)}{m_{b}^{0}}-\frac{\frac{1}{m_{b}-1} \sum_{j=1}^{m_{b}}\left(D_{j b}(1)-D_{j b}(0)\right)^{2}}{m_{b}} \\
&= \frac{\frac{1}{m_{b}-1} \sum_{j=1}^{m_{b}}\left(w_{j b}\left(\bar{Y}_{j b}(1)-\overline{\bar{Y}}_{b}(1)\right) / \bar{w}_{b}\right)^{2}}{m_{b}^{1}}+\frac{\frac{1}{m_{b}-1} \sum_{j=1}^{m_{b}}\left(w_{j b}\left(\bar{Y}_{j b}(0)-\overline{\bar{Y}}_{b}(0)\right) / \bar{w}_{b}\right)^{2}}{m_{b}^{0}} \\
&-\frac{\frac{1}{m_{b}-1} \sum_{j=1}^{m_{b}}\left(w_{j b}\left(\bar{Y}_{j b}(1)-\overline{\bar{Y}}_{b}(1)\right) / \bar{w}_{b}-w_{j b}\left(\bar{Y}_{j b}(0)-\overline{\bar{Y}}_{b}(0)\right) / \bar{w}_{b}\right)^{2}}{m_{b}} \\
&= \frac{1}{m_{b}-1} \sum_{j=1}^{m_{b}} \frac{w_{j b}^{2}}{\bar{w}_{b}^{2}}\left(\bar{Y}_{j b}(1)-\overline{\bar{Y}}_{b}(1)\right)^{2} \\
& m_{b}^{1}\left.\frac{1}{m_{b}-1} \sum_{j=1}^{m_{b}} \frac{w_{j b}^{2}}{\bar{w}_{b}^{2}}\left(\bar{Y}_{j b}(0)-\overline{\bar{Y}}_{b}(0)\right)\right)^{2} \\
& m_{b}^{0} \\
&-\frac{\frac{1}{m_{b}-1} \sum_{j=1}^{m_{b}} \frac{w_{j b}^{2}}{\bar{w}_{b}^{2}}\left(\bar{Y}_{j b}(1)-\bar{Y}_{j b}(0)-\left(\overline{\bar{Y}}_{b}(1)-\overline{\bar{Y}}_{b}(0)\right)\right)^{2}}{m_{b}} .
\end{aligned}
$$

\section{A.3 Theorem 1 with covariates, multiple blocks}

In this section we explore the impact estimator from a weighted least squares regression with additional covariate adjustment. We start by deriving the closed form of this estimator, which is $\hat{\beta}_{1, b}=\overline{\bar{y}}_{b}(1)-\overline{\bar{y}}_{b}(0)-\left(\overline{\overline{\boldsymbol{x}}}_{b}^{1}-\overline{\overline{\boldsymbol{x}}}_{b}^{0}\right) \hat{\boldsymbol{\gamma}}$ for block $b$. We then find consistency results and show the asymptotic normality result given in Theorem 1 . We do not specifically include interactions between the additional covariates and the block indicators as this immediately follows by generating the fully interacted set of covariates and then using our results on that extended set.

\section{A.3.1 The estimator}

We now have multiple blocks with covariate adjustment but not interactions between blocks and additional covariates in our regression. However, we continue to include an interaction between block and treatment. We assume that $m_{b} / m \stackrel{p}{\rightarrow} q_{b}$ where $0<q_{b}<1$. Let there be $h$ blocks. Accordingly, $\hat{\boldsymbol{\beta}}_{1}$ and $\hat{\boldsymbol{\beta}}_{0}$ are vectors with an entry for each block. Thus we have $\hat{\beta}_{1, b}$, the $b$ th entry of $\hat{\boldsymbol{\beta}}_{1}$, is still the treatment effect estimator for block $b$.

Now let $\tilde{\boldsymbol{x}}_{i j b}=\boldsymbol{x}_{i j b}-\overline{\overline{\boldsymbol{x}}}_{b}$ with

$$
\overline{\overline{\boldsymbol{x}}}_{b}=\frac{1}{w_{b}} \sum_{j=1}^{m_{b}} \sum_{i=1}^{n_{j b}} w_{i j b} \boldsymbol{x}_{i j b}
$$

and $\tilde{\overline{\boldsymbol{x}}}_{j b}=\overline{\boldsymbol{x}}_{j b}-\overline{\overline{\boldsymbol{x}}}_{b}$ with

$$
\overline{\boldsymbol{x}}_{j b}=\frac{1}{w_{j b}} \sum_{i=1}^{n_{j b}} w_{i j b} \boldsymbol{x}_{i j b}
$$


We also define

$$
\overline{\overline{\boldsymbol{x}}}_{b}^{1}=\frac{\sum_{j=1}^{m_{b}} w_{j b} T_{j b} \overline{\boldsymbol{x}}_{j b}}{\sum_{j=1}^{m_{b}} w_{j b} T_{j b}}
$$

and

$$
\overline{\overline{\boldsymbol{x}}}_{b}^{0}=\frac{\sum_{j=1}^{m_{b}} w_{j b}\left(1-T_{j b}\right) \overline{\boldsymbol{x}}_{j b}}{\sum_{j=1}^{m_{b}} w_{j b}\left(1-T_{j b}\right)} .
$$

We have $\boldsymbol{z}_{i j b}=\left(S_{i j 1} \tilde{T}_{j 1}, \ldots, S_{i j h} \tilde{T}_{j h}, S_{i j 1}, \ldots, S_{i j h}, \tilde{\boldsymbol{x}}_{i j b}\right)$. The estimated parameter vector is

$$
\left(\begin{array}{l}
\hat{\boldsymbol{\beta}}_{1} \\
\hat{\boldsymbol{\beta}}_{0}
\end{array}\right)=\left[\left(\sum_{b=1}^{h} \sum_{j=1}^{m_{b}} \sum_{i=1}^{n_{j b}} w_{i j b} \boldsymbol{z}_{i j b}^{\prime} \boldsymbol{z}_{i j b}\right)^{-1} \sum_{b=1}^{h} \sum_{j=1}^{m_{b}} \sum_{i=1}^{n_{j b}} w_{i j b} \boldsymbol{z}_{i j b}^{\prime} y_{i j b}\right] .
$$

\section{Result:}

$$
\hat{\beta}_{1, b}=\overline{\bar{y}}_{b}(1)-\overline{\bar{y}}_{b}(0)-\left(\overline{\overline{\boldsymbol{x}}}_{b}^{1}-\overline{\overline{\boldsymbol{x}}}_{b}^{0}\right) \hat{\boldsymbol{\gamma}}
$$

Remark. When we have interactions between blocks and additional covariates, we will have a different $\hat{\gamma}$ for each block and the estimators for each block will be independent. In that case, $\hat{\beta}_{1, b}$ is the same as the estimator as if we had only run the regression with block $b$, i.e. as if we only had one block. Therefore, these results directly extend to that case.

We have

$$
\begin{aligned}
& \left(\begin{array}{c}
\hat{\boldsymbol{\beta}}_{1} \\
\hat{\boldsymbol{\beta}}_{0} \\
\hat{\boldsymbol{\gamma}}
\end{array}\right) \\
& =\left(\begin{array}{ccccccc}
\sum_{j=1}^{m_{1}} w_{j 1} \tilde{T}_{j 1}^{2} & \cdots & 0 & 0 & \cdots & 0 & \sum_{j=1}^{m_{1}} w_{j 1} \tilde{T}_{j 1} \tilde{\overline{\boldsymbol{x}}}_{j 1} \\
\vdots & \ddots & \vdots & \vdots & \ddots & \vdots & \vdots \\
0 & \cdots & \sum_{j=1}^{m_{h}} w_{j h} \tilde{T}_{j h}^{2} & 0 & \cdots & 0 & \sum_{j=1}^{m_{h}} w_{j h} \tilde{T}_{j h} \tilde{\overline{\boldsymbol{x}}}_{j h} \\
0 & \cdots & 0 & w_{1} & \cdots & 0 & \mathbf{0}_{v} \\
\vdots & \ddots & \vdots & \vdots & \ddots & \vdots & \mathbf{0}_{v} \\
0 & \cdots & 0 & 0 & \cdots & w_{h} & \mathbf{0}_{v} \\
\sum_{j=1}^{m_{1}} w_{j 1} \tilde{T}_{j 1} \tilde{\overline{\boldsymbol{x}}}_{j 1}^{\prime} & \cdots & \sum_{j=1}^{m_{h}} w_{j h} \tilde{T}_{j h} \tilde{\overline{\boldsymbol{x}}}_{j h}^{\prime} & \mathbf{0}_{v}^{\prime} & \cdots & \mathbf{0}_{v}^{\prime} & \sum_{b=1}^{h} \sum_{j=1}^{m_{b}} \sum_{i=1}^{n_{j b}} w_{i j b} \tilde{\boldsymbol{x}}_{i j b}^{\prime} \tilde{\boldsymbol{x}}_{i j b}
\end{array}\right)^{-1} \\
& \times\left(\begin{array}{c}
\sum_{j=1}^{m_{1}} w_{j 1} \tilde{T}_{j 1} \bar{y}_{j 1} \\
\vdots \\
\sum_{j=1}^{m_{h}} w_{j h} \tilde{T}_{j h} \bar{y}_{j h} \\
\sum_{j=1}^{m_{1}} w_{j 1} \bar{y}_{j 1} \\
\vdots \\
\sum_{j=1}^{m_{h}} w_{j h} \bar{y}_{j h} \\
\sum_{b=1}^{h} \sum_{j=1}^{m_{b}} \sum_{i=1}^{n_{j b}} w_{i j b} \tilde{\boldsymbol{x}}_{i j b}^{\prime} y_{i j b}
\end{array}\right) .
\end{aligned}
$$


We start by performing the matrix inversion. We can break this matrix into the following blocks:

$$
\begin{aligned}
& \boldsymbol{A}=\left(\begin{array}{cccccc}
\sum_{j=1}^{m_{1}} w_{j 1} \tilde{T}_{j 1}^{2} & \cdots & 0 & 0 & \cdots & 0 \\
\vdots & \ddots & \vdots & \vdots & \ddots & \vdots \\
0 & \cdots & \sum_{j=1}^{m_{h}} w_{j h} \tilde{T}_{j h}^{2} & 0 & \cdots & 0 \\
0 & \cdots & 0 & w_{1} & \cdots & 0 \\
\vdots & \ddots & \vdots & \vdots & \ddots & \vdots \\
0 & \cdots & 0 & 0 & \cdots & w_{h}
\end{array}\right) \\
& =\left(\begin{array}{cccccc}
\frac{w_{1}^{1} w_{1}^{0}}{w_{1}} & \cdots & 0 & 0 & \cdots & 0 \\
\vdots & \ddots & \vdots & \vdots & \ddots & \vdots \\
0 & \cdots & \frac{w_{h}^{1} w_{h}^{0}}{w_{h}} & 0 & \cdots & 0 \\
0 & \cdots & 0 & w_{1} & \cdots & 0 \\
\vdots & \ddots & \vdots & \vdots & \ddots & \vdots \\
0 & \cdots & 0 & 0 & \cdots & w_{h}
\end{array}\right) \\
& \boldsymbol{B}=\left(\begin{array}{c}
\sum_{j=1}^{m_{1}} w_{j 1} \tilde{T}_{j 1} \tilde{\overline{\boldsymbol{x}}}_{j 1} \\
\vdots \\
\sum_{j=1}^{m_{h}} w_{j h} \tilde{T}_{j h} \tilde{\overline{\boldsymbol{x}}}_{j h} \\
\mathbf{0}_{v} \\
\vdots \\
\mathbf{0}_{v}
\end{array}\right) \\
& =\left(\begin{array}{c}
\frac{w_{1}^{1} w_{1}^{0}}{w_{1}}\left(\overline{\overline{\boldsymbol{x}}}_{1}^{1}-\overline{\overline{\boldsymbol{x}}}_{1}^{0}\right) \\
\vdots \\
\frac{w_{h}^{1} w_{h}^{0}}{w_{h}}\left(\overline{\overline{\boldsymbol{x}}}_{h}^{1}-\overline{\overline{\boldsymbol{x}}}_{h}^{0}\right) \\
\mathbf{0}_{v} \\
\vdots \\
\mathbf{0}_{v}
\end{array}\right)
\end{aligned}
$$

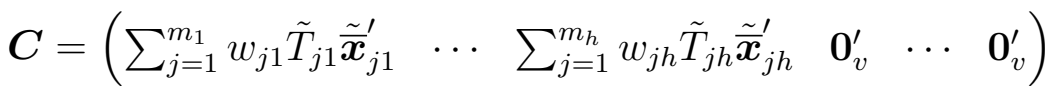

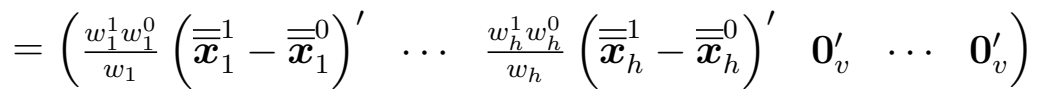

$$
\begin{aligned}
& \boldsymbol{D}=\left(\sum_{b=1}^{h} \sum_{j=1}^{m_{b}} \sum_{i=1}^{n_{j b}} w_{i j b} \tilde{\boldsymbol{x}}_{i j b}^{\prime} \tilde{\boldsymbol{x}}_{i j b}\right) \text {. }
\end{aligned}
$$

We can then use the following matrix inversion formula:

$$
\left(\begin{array}{ll}
\boldsymbol{A} & \boldsymbol{B} \\
\boldsymbol{C} & \boldsymbol{D}
\end{array}\right)^{-1}=\left(\begin{array}{cc}
\left(\boldsymbol{A}-\boldsymbol{B} \boldsymbol{D}^{-1} \boldsymbol{C}\right)^{-1} & -\boldsymbol{A}^{-1} \boldsymbol{B}\left(\boldsymbol{D}-\boldsymbol{C A}^{-1} \boldsymbol{B}\right)^{-1} \\
-\boldsymbol{D}^{-1} \boldsymbol{C}\left(\boldsymbol{A}-\boldsymbol{B} \boldsymbol{D}^{-1} \boldsymbol{C}\right)^{-1} & \left(\boldsymbol{D}-\boldsymbol{C} \boldsymbol{A}^{-1} \boldsymbol{B}\right)^{-1}
\end{array}\right) .
$$


To make things easier, we will first derive a result for

$$
\hat{\boldsymbol{\beta}}_{1}+\left(\begin{array}{c}
\overline{\overline{\boldsymbol{x}}}_{1}^{1}-\overline{\overline{\boldsymbol{x}}}_{1}^{0} \\
\vdots \\
\overline{\overline{\boldsymbol{x}}}_{h}^{1}-\overline{\overline{\boldsymbol{x}}}_{h}^{0}
\end{array}\right) \hat{\boldsymbol{\gamma}}
$$

We have, based on our matrix inversion, that

$$
\begin{aligned}
& \hat{\boldsymbol{\beta}}_{1}=\left(\begin{array}{cccccc}
1 & \cdots & 0 & 0 & \cdots & 0 \\
\vdots & \ddots & \vdots & \vdots & \ddots & \vdots \\
0 & \cdots & 1 & 0 & \cdots & 0
\end{array}\right)\left(\left(\boldsymbol{A}-\boldsymbol{B} \boldsymbol{D}^{-1} \boldsymbol{C}\right)^{-1}-\boldsymbol{A}^{-1} \boldsymbol{B}\left(\boldsymbol{D}-\boldsymbol{C A}^{-1} \boldsymbol{B}\right)^{-1}\right) \\
& \times\left(\begin{array}{c}
\sum_{j=1}^{m_{1}} w_{j 1} \tilde{T}_{j 1} \bar{y}_{j 1} \\
\vdots \\
\sum_{j=1}^{m_{h}} w_{j h} \tilde{T}_{j h} \bar{y}_{j h} \\
\sum_{j=1}^{m_{1}} w_{j 1} \bar{y}_{j 1} \\
\vdots \\
\sum_{j=1}^{m_{h}} w_{j h} \bar{y}_{j h} \\
\sum_{b=1}^{h} \sum_{j=1}^{m_{b}} \sum_{i=1}^{n_{j b}} w_{i j b} \tilde{\boldsymbol{x}}_{i j b}^{\prime} y_{i j b}
\end{array}\right) \\
& =\left(\begin{array}{cccccc}
1 & \cdots & 0 & 0 & \cdots & 0 \\
\vdots & \ddots & \vdots & \vdots & \ddots & \vdots \\
0 & \cdots & 1 & 0 & \cdots & 0
\end{array}\right)\left(\left(\boldsymbol{A}-\boldsymbol{B} \boldsymbol{D}^{-1} \boldsymbol{C}\right)^{-1}-\boldsymbol{A}^{-1} \boldsymbol{B}\left(\boldsymbol{D}-\boldsymbol{C A}^{-1} \boldsymbol{B}\right)^{-1}\right) \\
& \times\left(\begin{array}{c}
\frac{w_{1}^{1} w_{1}^{0}}{w_{1}}\left(\overline{\bar{y}}_{1}(1)-\overline{\bar{y}}_{1}(0)\right) \\
\vdots \\
\frac{w_{h}^{1} w_{h}^{0}}{w_{h}}\left(\overline{\bar{y}}_{h}(1)-\overline{\bar{y}}_{h}(0)\right) \\
\sum_{j=1}^{m_{1}} w_{j 1} \bar{y}_{j 1} \\
\vdots \\
\sum_{j=1}^{m_{h}} w_{j h} \bar{y}_{j h} \\
\sum_{b=1}^{h} \sum_{j=1}^{m_{b}} \sum_{i=1}^{n_{j b}} w_{i j b} \tilde{\boldsymbol{x}}_{i j b}^{\prime} y_{i j b}
\end{array}\right)
\end{aligned}
$$

and

$$
\begin{aligned}
& \left(\begin{array}{c}
\overline{\overline{\boldsymbol{x}}}_{1}^{1}-\overline{\overline{\boldsymbol{x}}}_{1}^{0} \\
\vdots \\
\overline{\overline{\boldsymbol{x}}}_{h}^{1}-\overline{\overline{\boldsymbol{x}}}_{h}^{0}
\end{array}\right) \hat{\gamma} \\
& =\left(\begin{array}{c}
\overline{\overline{\boldsymbol{x}}}_{1}^{1}-\overline{\overline{\boldsymbol{x}}}_{1}^{0} \\
\vdots \\
\overline{\overline{\boldsymbol{x}}}_{h}^{1}-\overline{\overline{\boldsymbol{x}}}_{h}^{0}
\end{array}\right)\left(-\boldsymbol{D}^{-1} \boldsymbol{C}\left(\boldsymbol{A}-\boldsymbol{B} \boldsymbol{D}^{-1} \boldsymbol{C}\right)^{-1} \quad\left(\boldsymbol{D}-\boldsymbol{C} \boldsymbol{A}^{-1} \boldsymbol{B}\right)^{-1}\right) \\
& \left(\begin{array}{c}
\frac{w_{1}^{1} w_{1}^{0}}{w_{1}}\left(\overline{\bar{y}}_{1}(1)-\overline{\bar{y}}_{1}(0)\right) \\
\vdots \\
\frac{w_{h}^{1} w_{h}^{0}}{w_{h}}\left(\overline{\bar{y}}_{h}(1)-\overline{\bar{y}}_{h}(0)\right) \\
\sum_{j=1}^{m_{1}} w_{j 1} \bar{y}_{j 1} \\
\vdots \\
\sum_{j=1}^{m_{h}} w_{j h} \bar{y}_{j h} \\
\sum_{b=1}^{h} \sum_{j=1}^{m_{b}} \sum_{i=1}^{n_{j b}} w_{i j b} \tilde{\boldsymbol{x}}_{i j b}^{\prime} y_{i j b}
\end{array}\right) .
\end{aligned}
$$


We see there are a lot of common terms when we add these expressions. Let's first simplify

$$
\begin{aligned}
& -\left(\begin{array}{cccccc}
1 & \cdots & 0 & 0 & \cdots & 0 \\
\vdots & \ddots & \vdots & \vdots & \ddots & \vdots \\
0 & \cdots & 1 & 0 & \cdots & 0
\end{array}\right) \boldsymbol{A}^{-1} \boldsymbol{B}+\left(\begin{array}{c}
\overline{\overline{\boldsymbol{x}}}_{1}^{1}-\overline{\overline{\boldsymbol{x}}}_{1}^{0} \\
\vdots \\
\overline{\overline{\boldsymbol{x}}}_{h}^{1}-\overline{\overline{\boldsymbol{x}}}_{h}^{0}
\end{array}\right) . \\
& \left(\begin{array}{cccccc}
1 & \cdots & 0 & 0 & \cdots & 0 \\
\vdots & \ddots & \vdots & \vdots & \ddots & \vdots \\
0 & \cdots & 1 & 0 & \cdots & 0
\end{array}\right) \boldsymbol{A}^{-1} \boldsymbol{B}=\left(\begin{array}{cccccc}
1 & \cdots & 0 & 0 & \cdots & 0 \\
\vdots & \ddots & \vdots & \vdots & \ddots & \vdots \\
0 & \cdots & 1 & 0 & \cdots & 0
\end{array}\right)\left(\begin{array}{ccccccc}
\frac{w_{1}}{w_{1}^{1} w_{1}^{0}} & \cdots & 0 & 0 & \cdots & 0 \\
\vdots & \ddots & \vdots & \vdots & \ddots & \vdots \\
0 & \cdots & \frac{w_{h}}{w_{h}^{h}} & 0 & \cdots & 0 \\
0 & \cdots & 0 & \frac{1}{w_{1}} & \cdots & 0 \\
\vdots & \ddots & \vdots & \vdots & \ddots & \vdots \\
0 & \cdots & 0 & 0 & \cdots & \frac{1}{w_{h}}
\end{array}\right) \\
& \times\left(\begin{array}{c}
\frac{w_{1}^{1} w_{1}^{0}}{w_{1}}\left(\overline{\overline{\boldsymbol{x}}}_{1}^{1}-\overline{\overline{\boldsymbol{x}}}_{1}^{0}\right) \\
\vdots \\
\frac{w_{h}^{1} w_{h}^{0}}{w_{h}}\left(\overline{\overline{\boldsymbol{x}}}_{h}^{1}-\overline{\overline{\boldsymbol{x}}}_{h}^{0}\right) \\
\mathbf{0}_{v} \\
\vdots \\
\mathbf{0}_{v}
\end{array}\right) \\
& =\left(\begin{array}{cccccc}
1 & \cdots & 0 & 0 & \cdots & 0 \\
\vdots & \ddots & \vdots & \vdots & \ddots & \vdots \\
0 & \cdots & 1 & 0 & \cdots & 0
\end{array}\right)\left(\begin{array}{c}
\overline{\overline{\boldsymbol{x}}}_{1}^{1}-\overline{\overline{\boldsymbol{x}}}_{1}^{0} \\
\vdots \\
\overline{\overline{\boldsymbol{x}}}_{h}^{1}-\overline{\overline{\boldsymbol{x}}}_{h}^{0} \\
\mathbf{0}_{v} \\
\vdots \\
\mathbf{0}_{v}
\end{array}\right) \\
& =\left(\begin{array}{c}
\overline{\overline{\boldsymbol{x}}}_{1}^{1}-\overline{\overline{\boldsymbol{x}}}_{1}^{0} \\
\vdots \\
\overline{\overline{\boldsymbol{x}}}_{h}^{1}-\overline{\overline{\boldsymbol{x}}}_{h}^{0}
\end{array}\right)
\end{aligned}
$$

We see that

$$
-\left(\begin{array}{cccccc}
1 & \cdots & 0 & 0 & \cdots & 0 \\
\vdots & \ddots & \vdots & \vdots & \ddots & \vdots \\
0 & \cdots & 1 & 0 & \cdots & 0
\end{array}\right) \boldsymbol{A}^{-1} \boldsymbol{B}+\left(\begin{array}{c}
\overline{\overline{\boldsymbol{x}}}_{1}^{1}-\overline{\overline{\boldsymbol{x}}}_{1}^{0} \\
\vdots \\
\overline{\overline{\boldsymbol{x}}}_{h}^{1}-\overline{\overline{\boldsymbol{x}}}_{h}^{0}
\end{array}\right)=\left(\begin{array}{c}
\mathbf{0}_{v} \\
\vdots \\
\mathbf{0}_{v}
\end{array}\right) .
$$

Now we need to simplify

$$
-\left(\begin{array}{cccccc}
1 & \cdots & 0 & 0 & \cdots & 0 \\
\vdots & \ddots & \vdots & \vdots & \ddots & \vdots \\
0 & \cdots & 1 & 0 & \cdots & 0
\end{array}\right)-\left(\begin{array}{c}
\overline{\overline{\boldsymbol{x}}}_{1}^{1}-\overline{\overline{\boldsymbol{x}}}_{1}^{0} \\
\vdots \\
\overline{\overline{\boldsymbol{x}}}_{h}^{1}-\overline{\overline{\boldsymbol{x}}}_{h}^{0}
\end{array}\right) \boldsymbol{D}^{-1} \boldsymbol{C} .
$$


But first let's look at $\boldsymbol{A}-\boldsymbol{B} \boldsymbol{D}^{-1} \boldsymbol{C}$.

$$
\begin{aligned}
& \boldsymbol{A}-\boldsymbol{B} \boldsymbol{D}^{-1} \boldsymbol{C}=\left(\begin{array}{cccccc}
\frac{w_{1}^{1} w_{1}^{0}}{w_{1}} & \cdots & 0 & 0 & \cdots & 0 \\
\vdots & \ddots & \vdots & \vdots & \ddots & \vdots \\
0 & \cdots & \frac{w_{h}^{1} w_{h}^{0}}{w_{h}} & 0 & \cdots & 0 \\
0 & \cdots & 0 & w_{1} & \cdots & 0 \\
\vdots & \ddots & \vdots & \vdots & \ddots & \vdots \\
0 & \cdots & 0 & 0 & \cdots & w_{h}
\end{array}\right)-\left(\begin{array}{c}
\frac{w_{1}^{1} w_{1}^{0}}{w_{1}}\left(\overline{\overline{\boldsymbol{x}}}_{1}^{1}-\overline{\boldsymbol{x}}_{1}^{0}\right) \\
\vdots \\
\frac{w_{h}^{1} w_{h}^{0}}{w_{h}}\left(\overline{\overline{\boldsymbol{x}}}_{h}^{1}-\overline{\overline{\boldsymbol{x}}}_{h}^{0}\right) \\
\mathbf{0}_{v} \\
\vdots \\
\mathbf{0}_{v}
\end{array}\right) \boldsymbol{D}^{-1} \boldsymbol{C} \\
& =\left(\begin{array}{cccccc}
\frac{w_{1}^{1} w_{1}^{0}}{w_{1}} & \cdots & 0 & 0 & \cdots & 0 \\
\vdots & \ddots & \vdots & \vdots & \ddots & \vdots \\
0 & \cdots & \frac{w_{h}^{1} w_{h}^{0}}{w_{h}} & 0 & \cdots & 0 \\
0 & \cdots & 0 & w_{1} & \cdots & 0 \\
\vdots & \ddots & \vdots & \vdots & \ddots & \vdots \\
0 & \cdots & 0 & 0 & \cdots & w_{h}
\end{array}\right)\left(\left(\begin{array}{cccccc}
1 & \cdots & 0 & 0 & \cdots & 0 \\
\vdots & \ddots & \vdots & \vdots & \ddots & \vdots \\
0 & \cdots & 1 & 0 & \cdots & 0 \\
0 & \cdots & 0 & 1 & \cdots & 0 \\
\vdots & \ddots & \vdots & \vdots & \ddots & \vdots \\
0 & \cdots & 0 & 0 & \cdots & 1
\end{array}\right)-\left(\begin{array}{c}
\left(\overline{\bar{x}}_{1}^{1}-\overline{\overline{\boldsymbol{x}}}_{1}^{0}\right) \\
\vdots \\
\left(\overline{\boldsymbol{x}}_{h}^{1}-\overline{\overline{\boldsymbol{x}}}_{h}^{0}\right) \\
\mathbf{0}_{v} \\
\vdots \\
\mathbf{0}_{v}
\end{array}\right) \boldsymbol{D}^{-1} \boldsymbol{C}\right)
\end{aligned}
$$

Now let's return to

$$
\left(\begin{array}{cccccc}
1 & \cdots & 0 & 0 & \cdots & 0 \\
\vdots & \ddots & \vdots & \vdots & \ddots & \vdots \\
0 & \cdots & 1 & 0 & \cdots & 0 \\
0 & \cdots & 0 & 1 & \cdots & 0 \\
\vdots & \ddots & \vdots & \vdots & \ddots & \vdots \\
0 & \cdots & 0 & 0 & \cdots & 1
\end{array}\right)-\left(\begin{array}{c}
\overline{\overline{\boldsymbol{x}}}_{1}^{1}-\overline{\overline{\boldsymbol{x}}}_{1}^{0} \\
\vdots \\
\overline{\overline{\boldsymbol{x}}}_{h}^{1}-\overline{\overline{\boldsymbol{x}}}_{h}^{0}
\end{array}\right) \boldsymbol{D}^{-1} \boldsymbol{C}
$$

$$
\left(\begin{array}{cccccc}
1 & \cdots & 0 & 0 & \cdots & 0 \\
\vdots & \ddots & \vdots & \vdots & \ddots & \vdots \\
0 & \cdots & 1 & 0 & \cdots & 0 \\
0 & \cdots & 0 & 1 & \cdots & 0 \\
\vdots & \ddots & \vdots & \vdots & \ddots & \vdots \\
0 & \cdots & 0 & 0 & \cdots & 1
\end{array}\right)-\left(\begin{array}{c}
\overline{\overline{\boldsymbol{x}}}_{1}^{1}-\overline{\overline{\boldsymbol{x}}}_{1}^{0} \\
\vdots \\
\overline{\boldsymbol{x}}_{h}^{1}-\overline{\overline{\boldsymbol{x}}}_{h}^{0}
\end{array}\right) \boldsymbol{D}^{-1} \boldsymbol{C}=\left(\begin{array}{cccccc}
\frac{w_{1}}{w_{1}^{1} w_{1}^{0}} & \cdots & 0 & 0 & \cdots & 0 \\
\vdots & \ddots & \vdots & \vdots & \ddots & \vdots \\
0 & \cdots & \frac{w_{2}}{w_{2}^{1} w_{2}^{0}} & 0 & \cdots & 0
\end{array}\right)\left(\boldsymbol{A}-\boldsymbol{B} \boldsymbol{D}^{-1} \boldsymbol{C}\right)
$$

Hence,

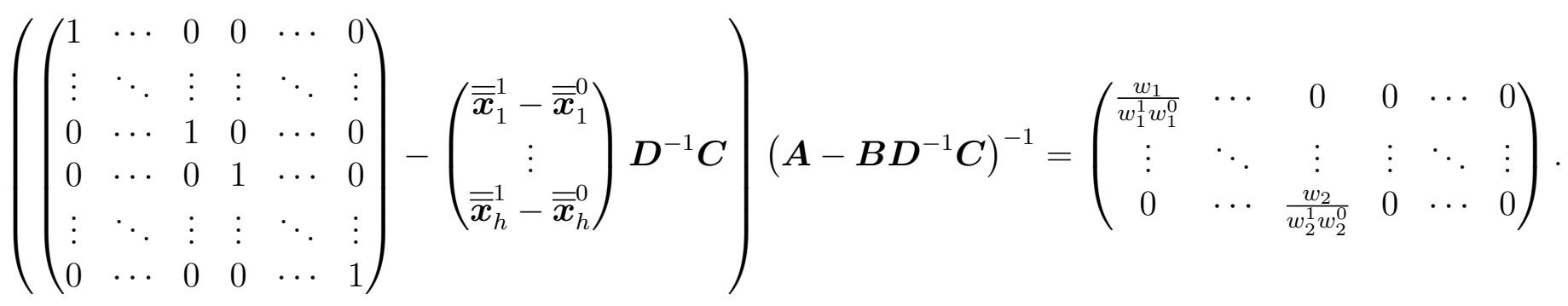


Putting it all together:

$$
\begin{aligned}
& \hat{\boldsymbol{\beta}}_{1}+\left(\begin{array}{c}
\overline{\overline{\boldsymbol{x}}}_{1}^{1}-\overline{\overline{\boldsymbol{x}}}_{1}^{0} \\
\vdots \\
\overline{\overline{\boldsymbol{x}}}_{h}^{1}-\overline{\overline{\boldsymbol{x}}}_{h}^{0}
\end{array}\right) \hat{\boldsymbol{\gamma}}=\left(\begin{array}{cccccc}
\frac{w_{1}}{w_{1}^{1} w_{1}^{0}} & \cdots & 0 & 0 & \cdots & 0 \\
\vdots & \ddots & \vdots & \vdots & \ddots & \vdots \\
0 & \cdots & \frac{w_{2}}{w_{2}^{1} w_{2}^{0}} & 0 & \cdots & 0
\end{array}\right)\left(\begin{array}{c}
\frac{w_{1}^{1} w_{1}^{0}}{w_{1}}\left(\overline{\bar{y}}_{1}(1)-\overline{\bar{y}}_{1}(0)\right) \\
\vdots \\
\frac{w_{h}^{1} w_{h}^{0}}{w_{h}}\left(\overline{\bar{y}}_{h}(1)-\overline{\bar{y}}_{h}(0)\right) \\
\sum_{j=1}^{m_{1}} w_{j 1} \bar{y}_{j 1} \\
\vdots \\
\sum_{j=1}^{m_{h}} w_{j h} \bar{y}_{j h} \\
\sum_{b=1}^{h} \sum_{j=1}^{m_{b}} \sum_{i=1}^{n_{j b}} w_{i j b} \tilde{\boldsymbol{x}}_{i j b}^{\prime} y_{i j b}
\end{array}\right) \\
&=\left(\begin{array}{c}
\overline{\bar{y}}_{1}(1)-\overline{\bar{y}}_{1}(0) \\
\vdots \\
\bar{y}_{h}(1)-\overline{\bar{y}}_{h}(0)
\end{array}\right) .
\end{aligned}
$$

Hence, we have the desired result,

$$
\hat{\boldsymbol{\beta}}_{1}=\left(\begin{array}{c}
\overline{\bar{y}}_{1}(1)-\overline{\bar{y}}_{1}(0) \\
\vdots \\
\overline{\bar{y}}_{h}(1)-\overline{\bar{y}}_{h}(0)
\end{array}\right)-\left(\begin{array}{c}
\overline{\overline{\boldsymbol{x}}}_{1}^{1}-\overline{\overline{\boldsymbol{x}}}_{1}^{0} \\
\vdots \\
\overline{\overline{\boldsymbol{x}}}_{h}^{1}-\overline{\overline{\boldsymbol{x}}}_{h}^{0}
\end{array}\right) \hat{\boldsymbol{\gamma}}
$$

\section{A.3.2 Consistency}

Here we will show that $\hat{\beta}_{1, b} \stackrel{p}{\rightarrow} \frac{1}{\omega_{b}}\left(\mu_{b}^{*}(1)-\mu_{b}^{*}(0)\right)=\beta_{1, b}^{*}$. We assume the same limiting values for our average potential outcomes and weights as in Section A.2.3. Assume that we have finite limiting values on the following weighted variance/covariance expressions, denoted as follows:

$$
\boldsymbol{S}_{\boldsymbol{x}, b}^{2}=\frac{1}{m_{b}} \sum_{j=1}^{m_{b}} \sum_{i=1}^{n_{j b}} w_{i j b}\left(\boldsymbol{x}_{i j b}-\overline{\overline{\boldsymbol{x}}}_{b}\right)^{\prime}\left(\boldsymbol{x}_{i j b}-\overline{\overline{\boldsymbol{x}}}_{b}\right) \stackrel{p}{\rightarrow} \boldsymbol{\Sigma}_{\boldsymbol{x}, b}^{2}
$$

and

$$
\boldsymbol{S}_{\boldsymbol{x}, Y, b}^{2}(t)=\frac{1}{m_{b}} \sum_{j=1}^{m_{b}} \sum_{i=1}^{n_{j b}} w_{i j b} \boldsymbol{x}_{i j b}^{\prime} Y_{i j b}(t)-\overline{\overline{\boldsymbol{x}}}_{b}^{\prime} \overline{w Y(t)} \stackrel{p}{\rightarrow} \boldsymbol{\Sigma}_{\boldsymbol{x}, Y(t), b}^{2} .
$$

Assume we also have a (unnamed) limiting value on the following variance expression:

$$
\boldsymbol{S}_{\boldsymbol{x} Y, b}^{2}(t)=\frac{1}{m_{b}} \sum_{j=1}^{m_{b}} \sum_{i=1}^{n_{j b}}\left(w_{i j b} \boldsymbol{x}_{i j b}^{\prime} Y_{i j b}(t)-\overline{w x Y(t)}\right)^{2}
$$

with

$$
\overline{w x Y(t)_{b}}=\frac{1}{m_{b}} \sum_{j=1}^{m_{b}} \sum_{i=1}^{n_{j b}} w_{i j b} \boldsymbol{x}_{i j b}^{\prime} Y_{i j b}(t) .
$$

Also assume that we have finite limiting values on the variances for the potential outcomes.

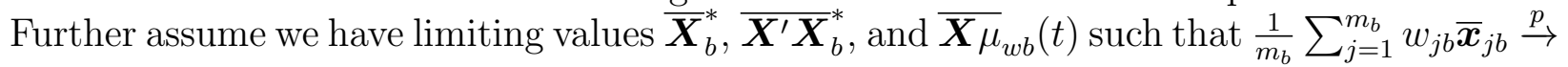
$\overline{\boldsymbol{X}}_{b}^{*}, \frac{1}{m_{b}} \sum_{j=1}^{m_{b}} \sum_{i=1}^{n_{j b}} w_{i j b} \boldsymbol{x}_{i j b}^{\prime} \boldsymbol{x}_{i j b} \stackrel{p}{\rightarrow} \overline{\boldsymbol{X}^{\prime} \boldsymbol{X}_{b}^{*}}$, and $\frac{1}{m_{b}} \sum_{j=1}^{m_{b}} \sum_{i=1}^{n_{j b}} w_{i j b} \tilde{\boldsymbol{x}}_{i j b}^{\prime} Y_{i j b}(t) \stackrel{p}{\rightarrow} \overline{\boldsymbol{X}}_{\mu_{b}}^{*}(t)$. Again, 
we assume that $m_{b} / m \stackrel{p}{\rightarrow} q_{b}$ where $0<q_{b}<1$, such that each block is growing to infinity with $m=\sum_{b=1}^{h} m_{b}$. In Section A.2.3, we already showed that $\overline{\bar{y}}_{b}(1)-\overline{\bar{y}}_{b}(0) \stackrel{p}{\rightarrow} \mu_{b}^{*}(1)-\mu_{b}^{*}(0)$. Thus, we now need to examine the extra term in this new $\hat{\beta}_{1, b}$, for which we need to simplify the asymptotic form of $\hat{\gamma}$.

$$
\begin{aligned}
& \left(\begin{array}{c}
\hat{\boldsymbol{\beta}}_{1} \\
\hat{\boldsymbol{\beta}}_{0} \\
\hat{\boldsymbol{\gamma}}
\end{array}\right) \\
& =\left(\begin{array}{ccccccc}
\sum_{j=1}^{m_{1}} w_{j 1} \tilde{T}_{j 1}^{2} & \cdots & 0 & 0 & \cdots & 0 & \sum_{j=1}^{m_{1}} w_{j 1} \tilde{T}_{j 1} \tilde{\boldsymbol{\boldsymbol { x }}}_{j 1} \\
\vdots & \ddots & \vdots & \vdots & \ddots & \vdots & \vdots \\
0 & \cdots & \sum_{j=1}^{m_{h}} w_{j h} \tilde{T}_{j h}^{2} & 0 & \cdots & 0 & \sum_{j=1}^{m_{h}} w_{j h} \tilde{T}_{j h} \tilde{\overline{\boldsymbol{x}}}_{j h} \\
0 & \cdots & 0 & w_{1} & \cdots & 0 & \mathbf{0}_{v} \\
\vdots & \ddots & \vdots & \vdots & \ddots & \vdots & \mathbf{0}_{v} \\
0 & \cdots & 0 & 0 & \cdots & w_{h} & \mathbf{0}_{v} \\
\sum_{j=1}^{m_{1}} w_{j 1} \tilde{T}_{j 1} \tilde{\overline{\boldsymbol{x}}}_{j 1}^{\prime} & \cdots & \sum_{j=1}^{m_{h}} w_{j h} \tilde{T}_{j h} \tilde{\overline{\boldsymbol{x}}}_{j h}^{\prime} & \mathbf{0}_{v}^{\prime} & \cdots & \mathbf{0}_{v}^{\prime} & \sum_{b=1}^{h} \sum_{j=1}^{m_{b}} \sum_{i=1}^{n_{j b}} w_{i j b} \tilde{\boldsymbol{x}}_{i j b}^{\prime} \tilde{\boldsymbol{x}}_{i j b}
\end{array}\right)^{-1} \\
& \times\left(\begin{array}{c}
\sum_{j=1}^{m_{1}} w_{j 1} \tilde{T}_{j 1} \bar{y}_{j 1} \\
\vdots \\
\sum_{j=1}^{m_{h}} w_{j h} \tilde{T}_{j h} \bar{y}_{j h} \\
\sum_{j=1}^{m_{1}} w_{j 1} \bar{y}_{j 1} \\
\vdots \\
\sum_{j=1}^{m_{h}} w_{j h} \bar{y}_{j h} \\
\sum_{b=1}^{h} \sum_{j=1}^{m_{b}} \sum_{i=1}^{n_{j b}} w_{i j b} \tilde{\boldsymbol{x}}_{i j b}^{\prime} y_{i j b}
\end{array}\right) \\
& =\left(\begin{array}{ccccccc}
\frac{1}{m_{1}} \sum_{j=1}^{m_{1}} w_{j 1} \tilde{T}_{j 1}^{2} & \cdots & 0 & 0 & \cdots & 0 & \frac{1}{m_{1}} \sum_{j=1}^{m_{1}} w_{j 1} \tilde{T}_{j 1} \tilde{\overline{\boldsymbol{x}}}_{j 1} \\
\vdots & \ddots & \vdots & \vdots & \ddots & \vdots & \vdots \\
0 & \cdots & \frac{1}{m_{h}} \sum_{j=1}^{m_{h}} w_{j h} \tilde{T}_{j h}^{2} & 0 & \cdots & 0 & \frac{1}{m_{h}} \sum_{j=1}^{m_{h}} w_{j h} \tilde{T}_{j h} \tilde{\overline{\boldsymbol{x}}}_{j h} \\
0 & \cdots & 0 & \frac{1}{m_{1}} w_{1} & \cdots & 0 & \mathbf{0}_{v} \\
\vdots & \ddots & \vdots & \vdots & \ddots & \vdots & \mathbf{0}_{v} \\
0 & \cdots & 0 & 0 & \cdots & \frac{1}{m_{h}} w_{h} & \mathbf{0}_{v} \\
\frac{1}{m} \sum_{j=1}^{m_{1}} w_{j 1} \tilde{T}_{j 1} \tilde{\overline{\boldsymbol{x}}}_{j 1}^{\prime} & \cdots & \frac{1}{m} \sum_{j=1}^{m_{h}} w_{j h} \tilde{T}_{j h} \tilde{\overline{\boldsymbol{x}}}_{j h}^{\prime} & \mathbf{0}_{v}^{\prime} & \cdots & \mathbf{0}_{v}^{\prime} & \frac{1}{m} \sum_{b=1}^{h} \sum_{j=1}^{m_{b}} \sum_{i=1}^{n_{j b}} w_{i j b} \tilde{\boldsymbol{x}}_{i j b}^{\prime} \tilde{\boldsymbol{x}}_{i j b}
\end{array}\right)^{-1} \\
& \times\left(\begin{array}{c}
\frac{1}{m_{1}} \sum_{j=1}^{m_{1}} w_{j 1} \tilde{T}_{j 1} \bar{y}_{j 1} \\
\vdots \\
\frac{1}{m_{h}} \sum_{j=1}^{m_{h}} w_{j h} \tilde{T}_{j h} \bar{y}_{j h} \\
\frac{1}{m_{1}} \sum_{j=1}^{m_{1}} w_{j 1} \bar{y}_{j 1} \\
\vdots \\
\frac{1}{m_{h}} \sum_{j=1}^{m_{h}} w_{j h} \bar{y}_{j h} \\
\frac{1}{m} \sum_{b=1}^{h} \sum_{j=1}^{m_{b}} \sum_{i=1}^{n_{j b}} w_{i j b} \tilde{\boldsymbol{x}}_{i j b}^{\prime} y_{i j b}
\end{array}\right)
\end{aligned}
$$


We have

$$
\begin{gathered}
\frac{1}{m_{b}} \sum_{j=1}^{m_{b}} w_{j b} \tilde{T}_{j b}^{2}=\frac{1}{m_{b}} \frac{w_{b}^{1} w_{b}^{0}}{w_{b}} \stackrel{p}{\rightarrow} p_{b}\left(1-p_{b}\right) \omega_{b}, \\
\frac{1}{m_{b}} \sum_{j=1}^{m_{b}} w_{j b} \stackrel{p}{\rightarrow} \omega_{b},
\end{gathered}
$$

and

$$
\frac{1}{m_{b}} \sum_{j=1}^{m_{b}} w_{j b} \tilde{T}_{j b} \tilde{\overline{\boldsymbol{x}}}_{j b}=\frac{1}{m_{b}} \frac{w_{b}^{0} w_{b}^{1}}{w_{b}}\left(\overline{\overline{\boldsymbol{x}}}_{b}^{1}-\overline{\overline{\boldsymbol{x}}}_{b}^{0}\right) \stackrel{p}{\rightarrow} 0
$$

because

$$
\overline{\overline{\boldsymbol{x}}}_{b}^{1}-\overline{\overline{\boldsymbol{x}}}_{b}^{0} \stackrel{p}{\rightarrow} \frac{\overline{\boldsymbol{X}}_{b}^{*}}{\omega_{b}}-\frac{\overline{\boldsymbol{X}}_{b}^{*}}{\omega_{b}}=0 .
$$

We also have

$$
\begin{aligned}
& \frac{1}{m} \sum_{b=1}^{h} \sum_{j=1}^{m_{b}} \sum_{i=1}^{n_{j b}} w_{i j b} \tilde{\boldsymbol{x}}_{i j b}^{\prime} \tilde{\boldsymbol{x}}_{i j b} \\
& =\frac{1}{m} \sum_{b=1}^{h} \sum_{j=1}^{m_{b}} \sum_{i=1}^{n_{j b}} w_{i j b}\left(\boldsymbol{x}_{i j b}-\overline{\overline{\boldsymbol{x}}}_{b}\right)^{\prime}\left(\boldsymbol{x}_{i j b}-\overline{\overline{\boldsymbol{x}}}_{b}\right) \\
& =\sum_{b=1}^{h} \frac{m_{b}}{m} \frac{1}{m_{b}} \sum_{j=1}^{m_{b}} \sum_{i=1}^{n_{j b}} w_{i j b}\left(\boldsymbol{x}_{i j b}-\overline{\overline{\boldsymbol{x}}}_{b}\right)^{\prime}\left(\boldsymbol{x}_{i j b}-\overline{\overline{\boldsymbol{x}}}_{b}\right) \\
& \stackrel{p}{\rightarrow} \sum_{b=1}^{h} q_{b} \boldsymbol{\Sigma}_{\boldsymbol{x}, b}^{2} .
\end{aligned}
$$

Then we have

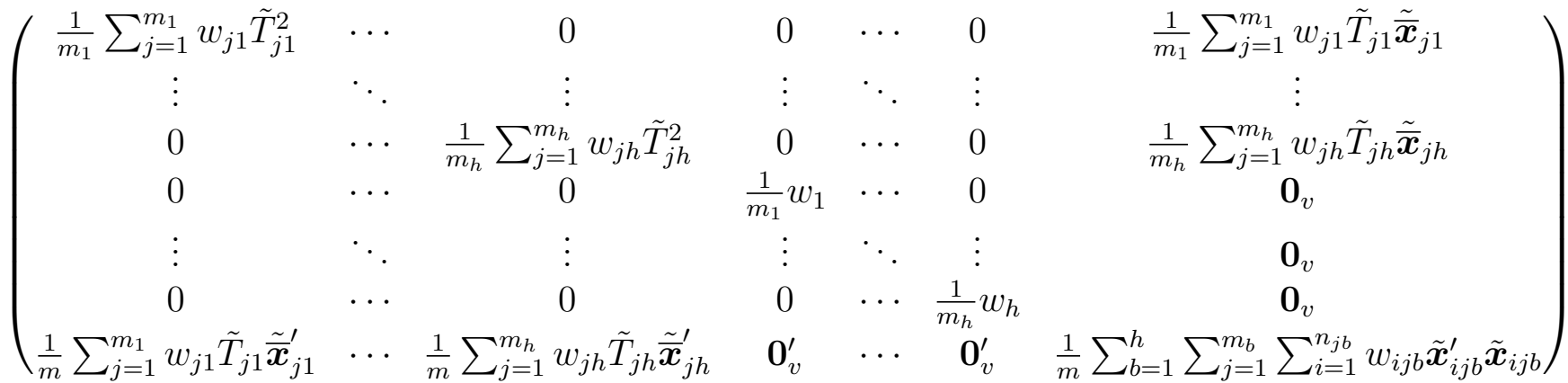

$$
\begin{aligned}
& \stackrel{p}{\rightarrow}\left(\begin{array}{ccccccc}
p_{1}\left(1-p_{1}\right) \omega_{1} & \cdots & 0 & 0 & \cdots & 0 & \mathbf{0}_{v} \\
\vdots & \ddots & \vdots & \vdots & \ddots & \vdots & \vdots \\
0 & \cdots & p_{h}\left(1-p_{h}\right) \omega_{h} & 0 & \cdots & 0 & \mathbf{0}_{v} \\
0 & \cdots & 0 & \omega_{1} & \cdots & 0 & \mathbf{0}_{v} \\
\vdots & \ddots & \vdots & \vdots & \ddots & \vdots & \vdots \\
0 & \cdots & 0 & 0 & \cdots & \omega_{h} & \mathbf{0}_{v} \\
\mathbf{0}_{v}^{\prime} & \cdots & \mathbf{0}_{v}^{\prime} & \mathbf{0}_{v}^{\prime} & \cdots & \mathbf{0}_{v}^{\prime} & \sum_{b=1}^{h} q_{b} \boldsymbol{\Sigma}_{\boldsymbol{x}, b}^{2}
\end{array}\right) .
\end{aligned}
$$


Hence, by continuity of the inverse and Slutsky's theorem,

$$
\begin{aligned}
& \left(\begin{array}{ccccccc}
\frac{1}{m_{1}} \sum_{j=1}^{m_{1}} w_{j 1} \tilde{T}_{j 1}^{2} & \cdots & 0 & 0 & \cdots & 0 & \frac{1}{m_{1}} \sum_{j=1}^{m_{1}} w_{j 1} \tilde{T}_{j 1} \tilde{\overline{\boldsymbol{x}}}_{j 1} \\
\vdots & \ddots & \vdots & \vdots & \ddots & \vdots & \vdots \\
0 & \cdots & \frac{1}{m_{h}} \sum_{j=1}^{m_{h}} w_{j h} \tilde{T}_{j h}^{2} & 0 & \cdots & 0 & \frac{1}{m_{h}} \sum_{j=1}^{m_{h}} w_{j h} \tilde{T}_{j h} \tilde{\overline{\boldsymbol{x}}}_{j h} \\
0 & \cdots & 0 & \frac{1}{m_{1}} w_{1} & \cdots & 0 & \mathbf{0}_{v} \\
\vdots & \ddots & \vdots & \vdots & \ddots & \vdots & \mathbf{0}_{v} \\
0 & \cdots & 0 & 0 & \cdots & \frac{1}{m_{h}} w_{h} & \mathbf{0}_{v} \\
\frac{1}{m} \sum_{j=1}^{m_{1}} w_{j 1} \tilde{T}_{j 1} \tilde{\overline{\boldsymbol{x}}}_{j 1}^{\prime} & \cdots & \frac{1}{m} \sum_{j=1}^{m_{h}} w_{j h} \tilde{T}_{j h} \tilde{\overline{\boldsymbol{x}}}_{j h}^{\prime} & \mathbf{0}_{v}^{\prime} & \cdots & \mathbf{0}_{v}^{\prime} & \frac{1}{m} \sum_{b=1}^{h} \sum_{j=1}^{m_{b}} \sum_{i=1}^{n_{j b}} w_{i j b} \tilde{\boldsymbol{x}}_{i j b}^{\prime} \tilde{\boldsymbol{x}}_{i j b}
\end{array}\right)^{-1} \\
& \stackrel{p}{\rightarrow}\left(\begin{array}{ccccccc}
\frac{1}{p_{1}\left(1-p_{1}\right) \omega_{1}} & \cdots & 0 & 0 & \cdots & 0 & \mathbf{0}_{v} \\
\vdots & \ddots & \vdots & \vdots & \ddots & \vdots & \vdots \\
0 & \cdots & \frac{1}{p_{h}\left(1-p_{h}\right) \omega_{h}} & 0 & \cdots & 0 & \mathbf{0}_{v} \\
0 & \cdots & 0 & \frac{1}{\omega_{1}} & \cdots & 0 & \mathbf{0}_{v} \\
\vdots & \ddots & \vdots & \vdots & \ddots & \vdots & \vdots \\
0 & \cdots & 0 & 0 & \cdots & \frac{1}{\omega_{h}} & \mathbf{0}_{v} \\
\mathbf{0}_{v}^{\prime} & \cdots & \mathbf{0}_{v}^{\prime} & \mathbf{0}_{v}^{\prime} & \cdots & \mathbf{0}_{v}^{\prime} & \left(\sum_{b=1}^{h} q_{b} \boldsymbol{\Sigma}_{\boldsymbol{x}, b}^{2}\right)^{-1}
\end{array}\right) .
\end{aligned}
$$

We also have

$$
\begin{aligned}
\frac{1}{m_{b}} \sum_{j=1}^{m_{b}} w_{j b} \tilde{T}_{j b} \bar{y}_{j b}=\frac{1}{m_{b}} \frac{w_{b}^{1} w_{b}^{0}}{w_{b}}\left(\overline{\bar{y}}_{b}(1)-\overline{\bar{y}}_{b}(0)\right) \stackrel{p}{\rightarrow} p_{b}\left(1-p_{b}\right) \omega_{b}\left(\frac{\mu_{b}^{*}(1)}{\omega_{b}}-\frac{\mu_{b}^{*}(0)}{\omega_{b}}\right) \\
=p_{b}\left(1-p_{b}\right)\left(\mu_{b}^{*}(1)-\mu_{b}^{*}(0)\right)
\end{aligned}
$$

and

$$
\frac{1}{m_{b}} \sum_{j=1}^{m_{b}} w_{j b} \bar{y}_{j b}=p_{b} \frac{1}{m_{b}^{1}} \sum_{j=1}^{m_{b}} w_{j b} T_{j b} \bar{Y}_{j b}(1)+\left(1-p_{b}\right) \frac{1}{m_{b}^{0}} \sum_{j=1}^{m_{b}} w_{j b}\left(1-T_{j b}\right) \bar{Y}_{j b}(0) \stackrel{p}{\rightarrow} p_{b} \mu_{b}^{*}(1)+\left(1-p_{b}\right) \mu_{b}^{*}(0) .
$$

Additionally,

$$
\begin{aligned}
& \frac{1}{m} \sum_{b=1}^{h} \sum_{j=1}^{m_{b}} \sum_{i=1}^{n_{j b}} w_{i j b} \tilde{\boldsymbol{x}}_{i j b}^{\prime} y_{i j b} \\
& =\frac{1}{m} \sum_{b=1}^{h} \sum_{j=1}^{m_{b}} \sum_{i=1}^{n_{j b}} T_{j b} w_{i j b}\left(\boldsymbol{x}_{i j b}-\overline{\boldsymbol{x}}_{b}\right) Y_{i j b}(1)+\frac{1}{m} \sum_{b=1}^{h} \sum_{j=1}^{m_{b}} \sum_{i=1}^{n_{j b}}\left(1-T_{j b}\right) w_{i j b}\left(\boldsymbol{x}_{i j b}-\overline{\boldsymbol{x}}_{b}\right) Y_{i j b}(0) \\
& =\sum_{b=1}^{h} \frac{m_{b}^{1}}{m}\left[\frac{1}{m_{b}^{1}} \sum_{j=1}^{m_{b}} \sum_{i=1}^{n_{j b}} T_{j b} w_{i j b} \boldsymbol{x}_{i j b} Y_{i j b}(1)-\overline{\overline{\boldsymbol{x}}}_{b}\left(\frac{1}{m_{b}^{1}} \sum_{j=1}^{m_{b}} T_{j b} w_{j b} \bar{Y}_{j b}(1)\right)\right] \\
& +\sum_{b=1}^{h} \frac{m_{b}^{0}}{m}\left[\frac{1}{m_{b}^{0}} \sum_{j=1}^{m_{b}} \sum_{i=1}^{n_{j b}}\left(1-T_{j b}\right) w_{i j b} \boldsymbol{x}_{i j b} Y_{i j b}(0)-\overline{\boldsymbol{x}}_{b}\left(\frac{1}{m_{b}^{0}} \sum_{j=1}^{m_{b}}\left(1-T_{j b}\right) w_{j b} \bar{Y}_{j b}(0)\right)\right] \\
& \stackrel{p}{\rightarrow} \sum_{b=1}^{h} p_{b} q_{b} \boldsymbol{\Sigma}_{\boldsymbol{x}, Y(1), b}^{2}+\sum_{b=1}^{h}\left(1-p_{b}\right) q_{b} \boldsymbol{\Sigma}_{\boldsymbol{x}, Y(0), b}^{2} .
\end{aligned}
$$


This last line comes from the following two intermediate steps:

(a) Because we have limiting values on the variances of our potential outcomes, we have a law of large numbers type result (see Theorem B of Scott and Wu (1981))

$$
\frac{1}{m_{b}^{1}} \sum_{j: T_{j b}=t} \sum_{i=1}^{n_{j b}} w_{i j b} \boldsymbol{x}_{i j b} Y_{i j b}(t)-\overline{\overline{\boldsymbol{x}}}_{b}\left(\frac{1}{m_{b}^{1}} \sum_{j: T_{j b}=t} w_{j b} \bar{Y}_{j b}(t)\right)-\boldsymbol{S}_{\boldsymbol{x}, Y, b}^{2}(t) \stackrel{p}{\rightarrow} 0 .
$$

(b) This implies (see Lemma A.2.1) that

$$
\frac{1}{m_{b}^{1}} \sum_{j: T_{j b}=t} \sum_{i=1}^{n_{j b}} w_{i j b} \boldsymbol{x}_{i j b} Y_{i j b}(t)-\overline{\overline{\boldsymbol{x}}}_{b}\left(\frac{1}{m_{b}^{1}} \sum_{j: T_{j b}=t} w_{j b} \bar{Y}_{j b}(t)\right) \stackrel{p}{\rightarrow} \boldsymbol{\Sigma}_{\boldsymbol{x}, Y(t), b}^{2} .
$$

Then we have

$$
\begin{gathered}
\left(\begin{array}{c}
\frac{1}{m_{1}} \sum_{j=1}^{m_{1}} w_{j 1} \tilde{T}_{j 1} \bar{y}_{j 1} \\
\vdots \\
\frac{1}{m_{h}} \sum_{j=1}^{m_{h}} w_{j h} \tilde{T}_{j h} \bar{y}_{j h} \\
\frac{1}{m_{1}} \sum_{j=1}^{m_{1}} w_{j 1} \bar{y}_{j 1} \\
\vdots \\
\frac{1}{m_{h}} \sum_{j=1}^{m_{h}} w_{j h} \bar{y}_{j h} \\
\frac{1}{m} \sum_{b=1}^{h} \sum_{j=1}^{m_{b}} \sum_{i=1}^{n_{j b}} w_{i j b} \tilde{\boldsymbol{x}}_{i j b}^{\prime} y_{i j b}
\end{array}\right) \\
p_{1}\left(1-p_{1}\right)\left(\mu_{1}^{*}(1)-\mu_{1}^{*}(0)\right) \\
\vdots \\
p_{h}\left(1-p_{h}\right)\left(\mu_{h}^{*}(1)-\mu_{h}^{*}(0)\right) \\
p_{1} \mu_{1}^{*}(1)+\left(1-p_{1}\right) \mu_{1}^{*}(0) \\
\vdots \\
p_{h} \mu_{h}^{*}(1)+\left(1-p_{h}\right) \mu_{h}^{*}(0) \\
\rightarrow\left(\begin{array}{c}
h \\
\sum_{b=1}^{h} p_{b} q_{b} \Sigma_{x, Y(1), b}^{2}+\sum_{b=1}^{h}\left(1-p_{b}\right) q_{b} \Sigma_{\boldsymbol{x}, Y(0), b}^{2}
\end{array}\right)
\end{gathered}
$$


Putting this all together, we have the following result:

$$
\begin{aligned}
& \left(\begin{array}{c}
\hat{\boldsymbol{\beta}}_{1} \\
\hat{\boldsymbol{\beta}}_{0} \\
\hat{\boldsymbol{\gamma}}
\end{array}\right) \stackrel{p}{\rightarrow}\left(\begin{array}{ccccccc}
\frac{1}{p_{1}\left(1-p_{1}\right) \omega_{1}} & \cdots & 0 & 0 & \cdots & 0 & \mathbf{0}_{v} \\
\vdots & \ddots & \vdots & \vdots & \ddots & \vdots & \vdots \\
0 & \cdots & \frac{1}{p_{h}\left(1-p_{h}\right) \omega_{h}} & 0 & \cdots & 0 & \mathbf{0}_{v} \\
0 & \cdots & 0 & \frac{1}{\omega_{1}} & \cdots & 0 & \mathbf{0}_{v} \\
\vdots & \ddots & \vdots & \vdots & \ddots & \vdots & \vdots \\
0 & \cdots & 0 & 0 & \cdots & \frac{1}{\omega_{h}} & \mathbf{0}_{v} \\
\mathbf{0}_{v}^{\prime} & \cdots & \mathbf{0}_{v}^{\prime} & \mathbf{0}_{v}^{\prime} & \cdots & \mathbf{0}_{v}^{\prime} & \left(\sum_{b=1}^{h} q_{b} \boldsymbol{\Sigma}_{\boldsymbol{x}, b}^{2}\right)^{-1}
\end{array}\right) \\
& \times\left(\begin{array}{c}
p_{1}\left(1-p_{1}\right)\left(\mu_{1}^{*}(1)-\mu_{1}^{*}(0)\right) \\
\vdots \\
p_{h}\left(1-p_{h}\right)\left(\mu_{h}^{*}(1)-\mu_{h}^{*}(0)\right) \\
p_{1} \mu_{1}^{*}(1)+\left(1-p_{1}\right) \mu_{1}^{*}(0) \\
\vdots \\
p_{h} \mu_{h}^{*}(1)+\left(1-p_{h}\right) \mu_{h}^{*}(0) \\
\sum_{b=1}^{h} p_{b} q_{b} \Sigma_{\boldsymbol{x}, Y(1), b}^{2}+\sum_{b=1}^{h}\left(1-p_{b}\right) q_{b} \Sigma_{\boldsymbol{x}, Y(0), b}^{2}
\end{array}\right) \\
& =\left(\begin{array}{c}
\frac{1}{\omega_{1}}\left(\mu_{1}^{*}(1)-\mu_{1}^{*}(0)\right) \\
\vdots \\
\frac{1}{\omega_{h}}\left(\mu_{h}^{*}(1)-\mu_{h}^{*}(0)\right) \\
\frac{1}{\omega_{1}}\left(p_{1} \mu_{1}^{*}(1)+\left(1-p_{1}\right) \mu_{1}^{*}(0)\right) \\
\vdots \\
\frac{1}{\omega_{h}}\left(p_{2} \mu_{h}^{*}(1)+\left(1-p_{h}\right) \mu_{h}^{*}(0)\right) \\
\Gamma
\end{array}\right) \\
& =\left(\begin{array}{c}
\beta_{11}^{*} \\
\vdots \\
\beta_{1 h}^{*} \\
\vdots \\
\frac{1}{\omega_{1}}\left(p_{1} \mu_{1}^{*}(1)+\left(1-p_{1}\right) \mu_{1}^{*}(0)\right) \\
\frac{1}{\omega_{h}}\left(p_{h} \mu_{h}^{*}(1)+\left(1-p_{h}\right) \mu_{h}^{*}(0)\right) \\
\Gamma
\end{array}\right),
\end{aligned}
$$

where

$$
\boldsymbol{\Gamma}=\left(\sum_{b=1}^{h} q_{b} \boldsymbol{\Sigma}_{\boldsymbol{x}, b}^{2}\right)^{-1}\left(\sum_{b=1}^{h} p_{b} q_{b} \boldsymbol{\Sigma}_{\boldsymbol{x}, Y(1), b}^{2}+\sum_{b=1}^{h}\left(1-p_{b}\right) q_{b} \boldsymbol{\Sigma}_{\boldsymbol{x}, Y(0), b}^{2}\right) .
$$

Hence, we have that $\hat{\beta}_{1, b} \stackrel{p}{\rightarrow} \beta_{1 b}^{*}$. 


\section{A.3.3 Asymptotic normality with known $\gamma$}

We ultimately want to find asymptotic normality results for the estimator for a single block $b$ with covariate adjustment across blocks, $\hat{\beta}_{1, b}=\overline{\bar{y}}_{b}(1)-\overline{\bar{y}}_{b}(0)-\left(\overline{\overline{\boldsymbol{x}}}_{b}^{1}-\overline{\overline{\boldsymbol{x}}}_{b}^{0}\right) \hat{\boldsymbol{\gamma}}$. But first, let us show, similar to $\mathrm{Li}$ and Ding (2017), that asymptotic normality holds for $\tilde{\beta}_{1 b}=$ $\overline{\bar{y}}_{b}(1)-\overline{\bar{y}}_{b}(0)-\left(\overline{\overline{\boldsymbol{x}}}_{b}^{1}-\overline{\overline{\boldsymbol{x}}}_{b}^{0}\right) \boldsymbol{\gamma}$, where $\boldsymbol{\gamma}$ is the finite population regression estimator we would have obtained if we had run the regression on the full schedule of potential outcomes, and is thus constant for each treatment arm. Stated differently, we first find the result when we know $\gamma$ and do not have to estimate it. In particular,

$$
\boldsymbol{\gamma}=\left(\sum_{b=1}^{h} q_{b} \boldsymbol{S}_{\boldsymbol{x}, b}^{2}\right)^{-1}\left(\sum_{b=1}^{h} p_{b} q_{b} \boldsymbol{S}_{\boldsymbol{x}, Y, b}^{2}(1)+\sum_{b=1}^{h}\left(1-p_{b}\right) q_{b} \boldsymbol{S}_{\boldsymbol{x}, Y, b}^{2}(0)\right)
$$

In this setting, we have that $C_{j b}(t)=\left(\bar{Y}_{j b}(t)-\overline{\boldsymbol{x}}_{j b} \boldsymbol{\gamma}\right)$ and so $D_{j b}(t)=w_{j b}\left(\bar{Y}_{j b}(t)-\overline{\boldsymbol{x}}_{j b} \boldsymbol{\gamma}-\right.$ $\left.\overline{\bar{Y}}_{b}(t)+\overline{\overline{\boldsymbol{x}}}_{b} \gamma\right) / \bar{w}_{b}$. Hence $\overline{\bar{c}}_{b}(t)=\overline{\bar{y}}_{b}(t)-\overline{\overline{\boldsymbol{x}}}_{b}^{1} \boldsymbol{\gamma}$ and $\overline{\bar{C}}_{b}(t)=\overline{\bar{Y}}_{b}(t)-\overline{\overline{\boldsymbol{x}}}_{b} \boldsymbol{\gamma}$.

Corollary A.3.1. Under the conditions of Lemma A.1.2 for block b,

$$
\begin{aligned}
\frac{\left(\overline{\bar{c}}_{b}(1)-\overline{\bar{c}}_{b}(0)\right)-\left(\overline{\bar{C}}_{b}(1)-\overline{\bar{C}}_{b}(0)\right)}{\sqrt{\operatorname{Var}\left(\hat{D}_{b}\right)}} & =\frac{\tilde{\beta}_{1 b}-\left(\overline{\bar{Y}}_{b}(1)-\overline{\bar{Y}}_{b}(0)\right)}{\sqrt{\operatorname{Var}\left(\hat{D}_{b}\right)}} \\
& \stackrel{d}{\rightarrow} N(0,1) .
\end{aligned}
$$

Proof. The result is a direct consequence of Lemma A.1.2. The equality holds by noting that

$$
\frac{\sum_{j=1}^{m_{b}} w_{j b}\left(\bar{Y}_{j b}(1)-\overline{\boldsymbol{x}}_{j b} \gamma\right)}{\sum_{j=1}^{m_{b}} w_{j b}}-\frac{\sum_{j=1}^{m_{b}} w_{j b}\left(\bar{Y}_{j b}(0)-\overline{\boldsymbol{x}}_{j b} \gamma\right)}{\sum_{j=1}^{m_{b}} w_{j b}}=\overline{\bar{Y}}_{b}(1)-\overline{\bar{Y}}_{b}(0)
$$


The variance in the denominator of our asymptotic expression simplifies as follows:

$$
\begin{aligned}
& \operatorname{Var}\left(\hat{D}_{b}\right)= \frac{S_{D, b}^{2}(1)}{m^{1}}+\frac{S_{D, b}^{2}(0)}{m^{0}}-\frac{S^{2}\left(D_{b}\right)}{m} \\
&= \frac{\frac{1}{m_{b}-1} \sum_{j=1}^{m_{b}} D_{j b}^{2}(1)}{m_{b}^{1}}+\frac{\frac{1}{m_{b}-1} \sum_{j=1}^{m_{b}} D_{j b}^{2}(0)}{m_{b}^{0}}-\frac{\frac{1}{m_{b}-1} \sum_{j=1}^{m_{b}}\left(D_{j b}(1)-D_{j b}(0)\right)^{2}}{m_{b}} \\
&= \frac{\frac{1}{m_{b}-1} \sum_{j=1}^{m_{b}} \frac{w_{j b}^{2}}{\bar{w}_{b}^{2}}\left(\bar{Y}_{j b}(1)-\overline{\bar{Y}}_{b}(1)-\left(\overline{\boldsymbol{x}}_{j b}-\overline{\overline{\boldsymbol{x}}}_{b}\right) \gamma\right)^{2}}{m_{b}^{1}} \\
&+\frac{\frac{1}{m_{b}-1} \sum_{j=1}^{m_{b}} \frac{w_{j b}^{2}}{\bar{w}_{b}^{2}}\left(\bar{Y}_{j b}(0)-\overline{\bar{Y}}_{b}(0)-\left(\overline{\boldsymbol{x}}_{j b}-\overline{\overline{\boldsymbol{x}}}_{b}\right) \gamma\right)^{2}}{m_{b}^{0}} \\
&-\frac{\frac{1}{m_{b}-1} \sum_{j=1}^{m_{b}} \frac{w_{j b}^{2}}{\bar{w}_{b}^{2}}\left(\bar{Y}_{j b}(1)-\bar{Y}_{j b}(0)-\left(\overline{\bar{Y}}_{b}(1)-\overline{\bar{Y}}_{b}(0)\right)\right)^{2}}{m_{b}} \\
& \frac{1}{m_{b}-1} \sum_{j=1}^{m_{b}} \frac{w_{j b}^{2}}{\bar{w}_{b}^{2}}\left(\bar{Y}_{j b}(1)-\overline{\bar{Y}}_{b}(1)-\tilde{\overline{\boldsymbol{x}}}_{j b} \gamma\right)^{2} \\
&-\frac{1}{m_{b}^{1}}+\frac{\sum_{b}-1}{m_{b}} \frac{w_{j b}^{2}}{\bar{w}_{b}^{2}}\left(\bar{Y}_{j b}(0)-\overline{\bar{Y}}_{b}(0)-\tilde{\overline{\boldsymbol{x}}}_{j b} \gamma\right)^{2} \\
& m_{b}-1 \\
& \sum_{j=1}^{m_{b}} \frac{w_{j b}^{2}}{\bar{w}_{b}^{2}}\left(\bar{Y}_{j b}(1)-\bar{Y}_{j b}(0)-\left(\overline{\bar{Y}}_{b}(1)-\overline{\bar{Y}}_{b}(0)\right)\right)^{2}
\end{aligned}
$$

\section{A.3.4 Joint asymptotic normality with known $\gamma$}

We now examine the joint convergence of $\tilde{\boldsymbol{\beta}}_{1}$. We see that each element of $\tilde{\boldsymbol{\beta}}_{1}, \tilde{\beta}_{1 b}$, is independent. Thus, we have the characteristic function for $A_{b}=\frac{\tilde{\beta}_{1 b}-\left(\overline{\bar{Y}}_{b}(1)-\overline{\bar{Y}}_{b}(0)\right)}{\sqrt{\operatorname{Var}\left(\hat{D}_{b}\right)}}$, which we denote $\phi_{b}(t)$, converges as follows:

$$
\phi_{b}(t) \rightarrow e^{-t^{2} / 2}
$$

by the equivalency of convergence in distribution and point-wise convergence of characteristic functions and using Corollary A.3.1.

Now take any linear combination $L$, defined as

$$
L=\sum_{b=1}^{h} s_{b} A_{b} .
$$


Then the characteristic function of $L$ is

$$
\begin{aligned}
\phi_{L}(t) & =E\left[e^{i t L}\right] \\
& =E\left[e^{i t \sum_{b=1}^{h} s_{b} A_{b}}\right] \\
& =E\left[\prod_{b=1}^{h} e^{i t s_{b} A_{b}}\right] \\
& =\prod_{b=1}^{h} E\left[e^{i t s_{b} A_{b}}\right] \\
& =\prod_{b=1}^{h} \phi_{b}\left(s_{b} t\right) \\
& \rightarrow \prod_{b=1}^{h} e^{-s_{b}^{2} t^{2} / 2} \\
& =e^{-\left(\sum_{b=1}^{h} s_{b}^{2}\right) t^{2} / 2} .
\end{aligned}
$$

Hence, $L \stackrel{d}{\rightarrow} N\left(0, \sum_{b=1}^{h} s_{b}^{2}\right)$. Therefore, by the Cramer-Wold device,

$$
\left(\begin{array}{c}
\frac{\tilde{\beta}_{11}-\left(\overline{\bar{Y}}_{1}(1)-\overline{\bar{Y}}_{1}(0)\right)}{\sqrt{\operatorname{Var}\left(\hat{D}_{1}\right)}} \\
\vdots \\
\frac{\tilde{\beta}_{1 h}-\left(\overline{\bar{Y}}_{h}(1)-\overline{\bar{Y}}_{h}(0)\right)}{\sqrt{\operatorname{Var}\left(\hat{D}_{h}\right)}}
\end{array}\right) \stackrel{d}{\rightarrow} N\left(\mathbf{0}_{h}, \boldsymbol{I}_{h}\right) \text {. }
$$

\section{A.3.5 Theorem 1: Asymptotic normality with estimated $\hat{\gamma}$}

We now move to our primary result, Theorem 1 . To obtain this result, we first find asymptotic normality results for $\hat{\beta}_{1, b}=\overline{\bar{y}}_{b}(1)-\overline{\bar{y}}_{b}(0)-\left(\overline{\overline{\boldsymbol{x}}}_{b}^{1}-\overline{\overline{\boldsymbol{x}}}_{b}^{0}\right) \hat{\boldsymbol{\gamma}}$ for a single block $b$. To do this, we need to show $\hat{\beta}_{1, b}$ has the same asymptotic distribution as $\tilde{\beta}_{1, b}=\overline{\bar{y}}_{b}(1)-\overline{\bar{y}}_{b}(0)-\left(\overline{\overline{\boldsymbol{x}}}_{b}^{1}-\overline{\overline{\boldsymbol{x}}}_{b}^{0}\right) \boldsymbol{\gamma}$, as done in Li and Ding (2017) for the unweighted case. Following that paper, we aim to show that the difference is order $o_{p}\left(m_{b}^{-1 / 2}\right)$.

First note that we can use results from Section A.2.4 to put a convergence rate on $\overline{\overline{\boldsymbol{x}}}_{b}^{1}-\overline{\overline{\boldsymbol{x}}}_{b}^{0}$. Convergence in probability of each element of $\overline{\boldsymbol{x}}_{b}^{1}$ and $\overline{\overline{\boldsymbol{x}}}_{b}^{0}$ implies convergence of the entire vector. Hence we can look at one entry of $\overline{\overline{\boldsymbol{x}}}_{b}^{1}$ and $\overline{\overline{\boldsymbol{x}}}_{b}^{0}$ at a time (since we can use componentwise convergence in probability). For the $k$ th component, let $C_{j b, k}(t)=\left[\overline{\boldsymbol{x}}_{j b}\right]_{k}$, noting that this does not change under treatment or control. Then under the conditions of Lemma A.1.1 we have asymptotic normality results for each of the components of $\overline{\boldsymbol{x}}_{b}^{1}$ and $\overline{\boldsymbol{x}}_{b}^{0}$. Assuming a limiting value on the variance, this in turn means that we have, for the $k$ th component, $\left[\overline{\overline{\boldsymbol{x}}}_{b}^{1}-\overline{\overline{\boldsymbol{x}}}_{b}\right]_{k}=O_{p}\left(m_{b}^{-1 / 2}\right)$ and $\left[\overline{\overline{\boldsymbol{x}}}_{b}^{0}-\overline{\overline{\boldsymbol{x}}}_{b}\right]_{k}=O_{p}\left(m_{b}^{-1 / 2}\right)$. 
Then we have

$$
\begin{aligned}
{\left[\overline{\overline{\boldsymbol{x}}}_{b}^{1}-\overline{\overline{\boldsymbol{x}}}_{b}^{0}\right]_{k} } & =\left[\overline{\overline{\boldsymbol{x}}}_{b}^{1}-\overline{\overline{\boldsymbol{x}}}_{b}-\left(\overline{\overline{\boldsymbol{x}}}_{b}^{0}-\overline{\overline{\boldsymbol{x}}}_{b}\right)\right]_{k} \\
& =O_{p}\left(m_{b}^{-1 / 2}\right) .
\end{aligned}
$$

Now note that

$$
\begin{aligned}
\hat{\beta}_{1, b} & =\overline{\bar{y}}_{b}(1)-\overline{\bar{y}}_{b}(0)-\left(\overline{\overline{\boldsymbol{x}}}_{b}^{1}-\overline{\overline{\boldsymbol{x}}}_{b}^{0}\right) \hat{\boldsymbol{\gamma}} \\
& =\frac{\sum_{j=1}^{m_{b}} T_{j b} w_{j b}\left(\bar{y}_{j b}-\overline{\boldsymbol{x}}_{j b} \boldsymbol{\gamma}\right)}{\sum_{j=1}^{m_{b}} T_{j b} w_{j b}}-\frac{\sum_{j=1}^{m_{b}}\left(1-T_{j b}\right) w_{j b}\left(\bar{y}_{j b}-\overline{\boldsymbol{x}}_{j b} \boldsymbol{\gamma}\right)}{\sum_{j=1}^{m_{b}}\left(1-T_{j b}\right) w_{j b}}-\left(\overline{\overline{\boldsymbol{x}}}_{b}^{1}-\overline{\overline{\boldsymbol{x}}}_{b}^{0}\right)(\hat{\boldsymbol{\gamma}}-\boldsymbol{\gamma}) .
\end{aligned}
$$

From the limiting value assumptions in Section A.3.2, $\hat{\boldsymbol{\gamma}}-\boldsymbol{\gamma} \stackrel{p}{\rightarrow} 0$ and so $\left(\overline{\overline{\boldsymbol{x}}}_{b}^{1}-\overline{\overline{\boldsymbol{x}}}_{b}^{0}\right)(\hat{\boldsymbol{\gamma}}-\boldsymbol{\gamma})=$ $o_{p}\left(m_{b}^{-1 / 2}\right)$. This means that $\hat{\beta}_{1, b}$ has the same asymptotic distribution as $\tilde{\beta}_{1 b}$ and so we can use Corollary A.3.1.

In Theorem 1, we assume the conditions of Corollary A.3.1, the conditions of Lemma A.1.1 applied to each of the components of $\overline{\boldsymbol{x}}_{b}^{1}$ and $\overline{\overline{\boldsymbol{x}}}_{b}^{0}$ as well as limiting values on the variance expression of each component. Also assume limiting values on the following variance expressions:

$$
\begin{aligned}
\boldsymbol{S}_{\boldsymbol{x}, b}^{2} & =\frac{1}{m_{b}} \sum_{j=1}^{m_{b}} \sum_{i=1}^{n_{j b}} w_{i j b}\left(\boldsymbol{x}_{i j b}-\overline{\overline{\boldsymbol{x}}}_{b}\right)^{\prime}\left(\boldsymbol{x}_{i j b}-\overline{\overline{\boldsymbol{x}}}_{b}\right) \\
\boldsymbol{S}_{\boldsymbol{x}, Y, b}^{2}(t) & =\frac{1}{m_{b}} \sum_{j=1}^{m_{b}} \sum_{i=1}^{n_{j b}} w_{i j b} \boldsymbol{x}_{i j b}^{\prime} Y_{i j b}(t)-\overline{\overline{\boldsymbol{x}}}_{b}^{\prime} \overline{w Y(t)_{b}} \\
\boldsymbol{S}_{\boldsymbol{x} Y, b}^{2}(t) & =\frac{1}{m_{b}} \sum_{j=1}^{m_{b}} \sum_{i=1}^{n_{j b}}\left(w_{i j b} \boldsymbol{x}_{i j b}^{\prime} Y_{i j b}(t)-\overline{w x Y(t)_{b}}\right)^{2} \text { for } t \in\{0,1\} \text { with } \\
\overline{w x Y(t)_{b}} & =\frac{1}{m_{b}} \sum_{j=1}^{m_{b}} \sum_{i=1}^{n_{j b}} w_{i j b} \boldsymbol{x}_{i j b}^{\prime} Y_{i j b}(t) .
\end{aligned}
$$

Then we have the result of Theorem 1 ,

$$
\frac{\hat{\beta}_{1, b}-\left(\overline{\bar{Y}}_{b}(1)-\overline{\bar{Y}}_{b}(0)\right)}{\sqrt{\operatorname{Var}\left(\hat{D}_{b}\right)}} \stackrel{d}{\rightarrow} N(0,1) .
$$

with

$$
\begin{aligned}
\operatorname{Var}\left(\hat{D}_{b}\right)= & \frac{\frac{1}{m_{b}-1} \sum_{j=1}^{m_{b}} \frac{w_{j b}^{2}}{\bar{w}_{b}^{2}}\left(\bar{Y}_{j b}(1)-\overline{\bar{Y}}_{b}(1)-\tilde{\overline{\boldsymbol{x}}}_{j b} \gamma\right)^{2}}{m_{b}^{1}}+\frac{\frac{1}{m_{b}-1} \sum_{j=1}^{m_{b}} \frac{w_{j b}^{2}}{\bar{w}_{b}^{2}}\left(\bar{Y}_{j b}(0)-\overline{\bar{Y}}_{b}(0)-\tilde{\overline{\boldsymbol{x}}}_{j b} \gamma\right)^{2}}{m_{b}^{0}} \\
& -\frac{\frac{1}{m_{b}-1} \sum_{j=1}^{m_{b}} \frac{w_{j b}^{2}}{\bar{w}_{b}^{2}}\left(\bar{Y}_{j b}(1)-\bar{Y}_{j b}(0)-\left(\overline{\bar{Y}}_{b}(1)-\overline{\bar{Y}}_{b}(0)\right)\right)^{2}}{m_{b}}
\end{aligned}
$$




\section{A.3.6 Joint asymptotic normality with estimated $\hat{\gamma}$}

We now investigate joint asymptotic normality results for the vector $\hat{\boldsymbol{\beta}}_{1}$. Each element of $\hat{\boldsymbol{\beta}}_{1}, \hat{\beta}_{1, b}$, is dependent because of the shared $\hat{\gamma}$ term.

With block-covariate interactions, each $\hat{\beta}_{1, b}$ is independent and thus the joint asymptotic result is immediate.

Without these interactions, we first define $C_{b}=\frac{\hat{\beta}_{1, b}-\left(\overline{\bar{Y}}_{b}(1)-\bar{Y}_{b}(0)\right)}{\sqrt{\operatorname{Var}\left(\hat{D}_{b}\right)}}$. Based on results from Section A.3.5, we have that $C_{b}$ converges to the same distribution that

$$
A_{b}=\frac{\tilde{\beta}_{1 b}-\left(\overline{\bar{Y}}_{b}(1)-\overline{\bar{Y}}_{b}(0)\right)}{\sqrt{\operatorname{Var}\left(\hat{D}_{b}\right)}}
$$

converges to. This implies that the linear combination

$$
S=\sum_{b=1}^{h} s_{b} C_{b}
$$

has the same asymptotic distribution as

$$
L=\sum_{b=1}^{h} s_{b} A_{b}
$$

In particular,

$$
S=\sum_{b=1}^{h} s_{b} \frac{\hat{\beta}_{1, b}-\left(\overline{\bar{Y}}_{b}(1)-\overline{\bar{Y}}_{b}(0)\right)}{\sqrt{\operatorname{Var}\left(\hat{D}_{b}\right)}}=\sum_{b=1}^{h} s_{b} A_{b}-\sum_{b=1}^{h} s_{b}\left(\overline{\overline{\boldsymbol{x}}}_{b}^{1}-\overline{\overline{\boldsymbol{x}}}_{b}^{0}\right)(\hat{\boldsymbol{\gamma}}-\boldsymbol{\gamma})
$$

and the last sum is a finite sum of terms that are $o_{p}\left(m_{b}^{-1 / 2}\right)$.

Hence, $S \stackrel{d}{\rightarrow} N\left(0, \sum_{b=1}^{h} s_{b}^{2}\right)$. Therefore, by the Cramer-Wold device,

$$
\left(\begin{array}{c}
\frac{\hat{\beta}_{1,1}-\left(\overline{\bar{Y}}_{1}(1)-\overline{\bar{Y}}_{1}(0)\right)}{\sqrt{\operatorname{Var}\left(\hat{D}_{1}\right)}} \\
\vdots \\
\frac{\hat{\beta}_{1, h}-\left(\overline{\bar{Y}}_{h}(1)-\overline{\bar{Y}}_{h}(0)\right)}{\sqrt{\operatorname{Var}\left(\hat{D}_{h}\right)}}
\end{array}\right) \stackrel{d}{\rightarrow} N\left(\mathbf{0}_{h}, \boldsymbol{I}_{h}\right) .
$$

\section{A.4 Restricted model}

In this section, we explore the impact estimator from a weighted least squares regression with additional covariate adjustment and without interactions between block indicators and treatment indicators. We now have a single treatment effect estimator that aggregates across blocks. We first find the closed form for this estimator as

$$
\hat{\beta}_{1}=\frac{\sum_{b=1}^{h} \frac{w_{b}^{1} w_{b}^{0}}{w_{b}}\left(\overline{\bar{y}}_{b}(1)-\overline{\bar{y}}_{b}(0)\right)}{\sum_{b=1}^{h} \frac{w_{b}^{1} w_{b}^{0}}{w_{b}}}-\frac{\sum_{b=1}^{h} \frac{w_{b}^{1} w_{b}^{0}}{w_{b}}\left(\overline{\overline{\boldsymbol{x}}}_{b}^{1}-\overline{\overline{\boldsymbol{x}}}_{b}^{0}\right)}{\sum_{b=1}^{h} \frac{w_{b}^{1} w_{b}^{0}}{w_{b}}} \hat{\boldsymbol{\gamma}}
$$


We then find consistency results and show an asymptotic normality result. As before, we first assume a known $\gamma$ to focus on the pooling of individual treatment impacts across blocks and then extend to an estimated $\hat{\gamma}$.

\section{A.4.1 The estimator}

We now examine the model with no interactions between treatment and blocks . In this case, there is a single treatment effect estimator, $\hat{\beta}_{1}$ for all blocks. We have $\boldsymbol{z}_{i j b}=\left(\tilde{T}_{j b}, S_{i j 1}, \ldots, S_{i j h}, \tilde{\boldsymbol{x}}_{i j b}\right)$. The estimated parameter vector is

$$
\left(\begin{array}{c}
\hat{\beta}_{1} \\
\hat{\boldsymbol{\beta}}_{0} \\
\hat{\boldsymbol{\gamma}}
\end{array}\right)=\left[\left(\sum_{b=1}^{h} \sum_{j=1}^{m_{b}} \sum_{i=1}^{n_{j b}} w_{i j b} \boldsymbol{z}_{i j b}^{\prime} \boldsymbol{z}_{i j b}\right)^{-1} \sum_{b=1}^{h} \sum_{j=1}^{m_{b}} \sum_{i=1}^{n_{j b}} w_{i j b} \boldsymbol{z}_{i j b}^{\prime} y_{i j b}\right] .
$$

Using the same techniques as in Section A.3.1 we find the following:

Result:

$$
\hat{\beta}_{1}=\frac{\sum_{b=1}^{h} \frac{w_{b}^{1} w_{b}^{0}}{w_{b}}\left(\overline{\bar{y}}_{b}(1)-\overline{\bar{y}}_{b}(0)\right)}{\sum_{b=1}^{h} \frac{w_{b}^{1} w_{b}^{0}}{w_{b}}}-\frac{\sum_{b=1}^{h} \frac{w_{b}^{1} w_{b}^{0}}{w_{b}}\left(\overline{\overline{\boldsymbol{x}}}_{b}^{1}-\overline{\overline{\boldsymbol{x}}}_{b}^{0}\right)}{\sum_{b=1}^{h} \frac{w_{b}^{1} w_{b}^{0}}{w_{b}}} \hat{\boldsymbol{\gamma}}
$$

Derivations available upon request.

\section{A.4.2 Consistency}

Result:

$$
\begin{aligned}
\hat{\beta}_{1} & =\frac{\sum_{b=1}^{h} \frac{w_{b}^{1} w_{b}^{0}}{w_{b}}\left(\overline{\bar{y}}_{b}(1)-\overline{\bar{y}}_{b}(0)\right)}{\sum_{b=1}^{h} \frac{w_{b}^{1} w_{b}^{0}}{w_{b}}}-\frac{\sum_{b=1}^{h} \frac{w_{b}^{1} w_{b}^{0}}{w_{b}}\left(\overline{\overline{\boldsymbol{x}}}_{b}^{1}-\overline{\overline{\boldsymbol{x}}}_{b}^{0}\right)}{\sum_{b=1}^{h} \frac{w_{b}^{1} w_{b}^{0}}{w_{b}}} \hat{\boldsymbol{\gamma}} \\
& \stackrel{p}{\rightarrow} \frac{\sum_{b=1}^{h} q_{b} p_{b}\left(1-p_{b}\right)\left(\mu_{b}^{*}(1)-\mu_{b}^{*}(0)\right)}{\sum_{b=1}^{h} q_{b} p_{b}\left(1-p_{b}\right) \omega_{b}}
\end{aligned}
$$

Using results and limiting values given in Section A.2.3 and Section A.3.2 and also 
$m_{b} / m \stackrel{p}{\rightarrow} q_{b}\left(0<q_{b}<1\right)$, we have

$$
\begin{aligned}
& \left(\begin{array}{c}
\hat{\beta}_{1} \\
\hat{\boldsymbol{\beta}}_{0} \\
\hat{\boldsymbol{\gamma}}
\end{array}\right)=\left(\begin{array}{ccccc}
\frac{1}{m} \sum_{b=1}^{h} \frac{w_{b}^{1} w_{b}^{0}}{w_{b}} & 0 & \cdots & 0 & \frac{1}{m} \sum_{b=1}^{h} \frac{w_{b}^{1} w_{b}^{0}}{w_{b}}\left(\overline{\overline{\boldsymbol{x}}}_{b}^{1}-\overline{\overline{\boldsymbol{x}}}_{b}^{0}\right) \\
0 & \frac{1}{m_{1}} w_{1} & \cdots & 0 & \mathbf{0}_{v} \\
\vdots & \vdots & \ddots & \vdots & \vdots \\
0 & 0 & \cdots & \frac{1}{m_{h}} w_{h} & \mathbf{0}_{v} \\
\frac{1}{m} \sum_{b=1}^{h} \frac{w_{b}^{1} w_{b}^{0}}{w_{b}}\left(\overline{\overline{\boldsymbol{x}}}_{b}^{1^{\prime}}-\overline{\overline{\boldsymbol{x}}}_{b}^{0^{\prime}}\right) & \mathbf{0}_{v}^{\prime} & \cdots & \mathbf{0}_{v}^{\prime} & \frac{1}{m} \sum_{b=1}^{h} \sum_{j=1}^{m_{b}} \sum_{i=1}^{n_{j b}} w_{i j b} \tilde{\boldsymbol{x}}_{i j b}^{\prime} \tilde{\boldsymbol{x}}_{i j b}
\end{array}\right)^{-1} \\
& \times\left(\begin{array}{c}
\frac{1}{m} \sum_{b=1}^{h} \frac{w_{b}^{1} w_{b}^{0}}{w_{b}}\left(\overline{\bar{y}}_{b}(1)-\overline{\bar{y}}_{b}(0)\right) \\
\frac{1}{m_{1}}\left(w_{1}^{1} \overline{\bar{y}}_{1}(1)+w_{1}^{0} \overline{\bar{y}}_{1}(0)\right) \\
\vdots \\
\frac{1}{m_{h}}\left(w_{h}^{1} \overline{\bar{y}}_{h}(1)+w_{h}^{0} \overline{\bar{y}}_{h}(0)\right) \\
\frac{1}{m} \sum_{b=1}^{h} \sum_{j=1}^{m_{b}} \sum_{i=1}^{n_{j b}} w_{i j b} \tilde{\boldsymbol{x}}_{i j b}^{\prime} \bar{y}_{i j b}
\end{array}\right) \\
& \stackrel{p}{\rightarrow}\left(\begin{array}{ccccc}
\sum_{b=1}^{h} q_{b} p_{b}\left(1-p_{b}\right) \omega_{b} & 0 & \cdots & 0 & 0 \\
0 & \omega_{1} & \cdots & 0 & \mathbf{0}_{v} \\
\vdots & \vdots & \ddots & \vdots & \vdots \\
0 & 0 & \cdots & \omega_{h} & \mathbf{0}_{v} \\
0 & \mathbf{0}_{v}^{\prime} & \cdots & \mathbf{0}_{v}^{\prime} & \sum_{b=1}^{h} q_{b} \boldsymbol{\Sigma}_{\boldsymbol{x}, b}^{2}
\end{array}\right)^{-1} \\
& \times\left(\begin{array}{c}
\sum_{b=1}^{h} q_{b} p_{b}\left(1-p_{b}\right)\left(\mu_{b}^{*}(1)-\mu_{b}^{*}(0)\right) \\
p_{1} \mu_{1}(1)+\left(1-p_{1}\right) \mu_{1}(0) \\
\vdots \\
p_{h} \mu_{h}(1)+\left(1-p_{h}\right) \mu_{h}(0) \\
\sum_{b=1}^{h} p_{b} q_{b} \Sigma_{\boldsymbol{x}, Y(1), b}^{2}+\sum_{b=1}^{h}\left(1-p_{b}\right) q_{b} \Sigma_{\boldsymbol{x}, Y(0), b}^{2}
\end{array}\right) \\
& =\left(\begin{array}{c}
\frac{\sum_{b=1}^{h} q_{b} p_{b}\left(1-p_{b}\right)\left(\mu_{b}^{*}(1)-\mu_{b}^{*}(0)\right)}{\sum_{b=1}^{h} q_{b} p_{b}\left(1-p_{b}\right) \omega_{b}} \\
\frac{1}{\omega_{1}}\left(p_{1} \mu_{1}(1)+\left(1-p_{1}\right) \mu_{1}(0)\right) \\
\vdots \\
\frac{1}{\omega_{h}}\left(p_{h} \mu_{h}(1)+\left(1-p_{h}\right) \mu_{h}(0)\right) \\
\Gamma
\end{array}\right)
\end{aligned}
$$

where

$$
\boldsymbol{\Gamma}=\left(\sum_{b=1}^{h} q_{b} \boldsymbol{\Sigma}_{\boldsymbol{x}, b}^{2}\right)^{-1}\left(\sum_{b=1}^{h} p_{b} q_{b} \boldsymbol{\Sigma}_{\boldsymbol{x}, Y(1), b}^{2}+\sum_{b=1}^{h}\left(1-p_{b}\right) q_{b} \boldsymbol{\Sigma}_{\boldsymbol{x}, Y(0), b}^{2}\right)
$$




\section{A.4.3 Asymptotic normality with known $\gamma$}

Let $\tilde{q}_{b}=m_{b} / m$. Following Section A.3.4, we want to start by finding the asymptotic distribution of

$$
\begin{aligned}
\tilde{\beta}_{1} & =\frac{\sum_{b=1}^{h} \frac{w_{b}^{1} w_{b}^{0}}{w_{b}}\left(\overline{\bar{y}}_{b}(1)-\overline{\bar{y}}_{b}(0)\right)}{\sum_{a=1}^{h} \frac{w_{a}^{1} w_{a}^{0}}{w_{a}}}-\frac{\sum_{b=1}^{h} \frac{w_{b}^{1} w_{b}^{0}}{w_{b}}\left(\overline{\overline{\boldsymbol{x}}}_{b}^{1}-\overline{\overline{\boldsymbol{x}}}_{b}^{0}\right)}{\sum_{a=1}^{h} \frac{w_{a}^{1} w_{a}^{0}}{w_{a}}} \gamma \\
& =\sum_{b=1}^{h} \frac{\tilde{q}_{b} p_{b} \bar{w}_{b}^{1}\left(\bar{w}_{b}-p_{b} \bar{w}_{b}^{1}\right) / \bar{w}_{b}}{\sum_{a=1}^{h} \tilde{q}_{a} p_{a} \bar{w}_{a}^{1}\left(\bar{w}_{a}-p_{a} \bar{w}_{a}^{1}\right) / \bar{w}_{a}}\left(\overline{\bar{y}}_{b}(1)-\overline{\bar{y}}_{b}(0)-\left(\overline{\overline{\boldsymbol{x}}}_{b}^{1}-\overline{\boldsymbol{x}}_{b}^{0}\right) \gamma\right) .
\end{aligned}
$$

Further denote

$$
\beta_{1}=\sum_{b=1}^{h} \frac{\tilde{q}_{b} p_{b}\left(1-p_{b}\right) \bar{w}_{b}}{\sum_{a=1}^{h} \tilde{q}_{a} p_{a}\left(1-p_{a}\right) \bar{w}_{a}}\left(\overline{\bar{Y}}_{b}(1)-\overline{\bar{Y}}_{b}(0)\right)
$$

Let

$$
U_{j b}(t)=w_{j b}\left(\bar{Y}_{j b}(t)-\overline{\boldsymbol{x}}_{j b} \gamma\right), \quad \bar{U}_{b}(t)=\frac{1}{m_{b}} \sum_{j=1}^{m_{b}} U_{j b}(t), \quad \text { and } \quad \bar{u}_{b}(t)=\frac{1}{m_{b}^{t}} \sum_{j: T_{j b}=t} U_{j b}(t) .
$$

Then we can write

$$
\tilde{\beta}_{1}=\sum_{b=1}^{h} \frac{\tilde{q}_{b} p_{b} \bar{w}_{b}^{1}\left(\bar{w}_{b}-p_{b} \bar{w}_{b}^{1}\right) / \bar{w}_{b}}{\sum_{a=1}^{h} \tilde{q}_{a} p_{a} \bar{w}_{a}^{1}\left(\bar{w}_{a}-p_{a} \bar{w}_{a}^{1}\right) / \bar{w}_{a}}\left(\frac{\bar{u}_{b}(1)}{\bar{w}^{1}}-\frac{\bar{u}_{b}(0)}{\bar{w}^{0}}\right) .
$$

It is useful to rewrite this in terms of the fewest possible random variables, so we rewrite $\bar{w}_{b}^{1}=\left(\bar{w}_{b}-p_{b} \bar{w}_{b}^{1}\right) /\left(1-p_{b}\right)$ everywhere as follows:

$$
\tilde{\beta}_{1}=\sum_{b=1}^{h} \frac{\tilde{q}_{b} p_{b} \bar{w}_{b}^{1}\left(\bar{w}_{b}-p_{b} \bar{w}_{b}^{1}\right) / \bar{w}_{b}}{\sum_{a=1}^{h} \tilde{q}_{a} p_{a} \bar{w}_{a}^{1}\left(\bar{w}_{a}-p_{a} \bar{w}_{a}^{1}\right) / \bar{w}_{a}}\left(\frac{\bar{u}_{b}(1)}{\bar{w}^{1}}-\frac{\left(1-p_{b}\right) \bar{u}_{b}(0)}{\bar{w}_{b}-p_{b} \bar{w}_{b}^{1}}\right) .
$$

Let, for $t \in\{0,1\}$ and $z \in\{w, U\}$,

$$
a_{z, b}(t)=\max _{1 \leq j \leq m_{b}}\left(z_{j, b}(t)-\bar{z}_{b}(t)\right)^{2}
$$

and

$$
v_{z, b}(t)=\frac{1}{m_{b}-1} \sum_{j=1}^{m_{b}}\left(z_{j, b}(t)-\bar{z}_{b}(t)\right)^{2},
$$

noting that $w_{j b}(t)=w_{j b}$. Let $\boldsymbol{t}=\left(\bar{u}_{1}(1), \bar{u}_{1}(0), \bar{w}_{1}^{1}, \cdots, \bar{u}_{h}(1), \bar{u}_{h}(0), \bar{w}_{h}^{1}\right)$ and $\boldsymbol{T}=\left(\bar{U}_{1}(1), \bar{U}_{1}(0), \bar{w}_{1}, \cdots, \bar{U}_{h}(1), \bar{U}_{h}(0), \bar{w}_{h}\right)$.

Theorem A.1. Let us assume the following conditions:

(a) As $m \rightarrow \infty$,

$$
\max _{1 \leq b \leq h} \max _{z \in\{w, U\}} \max _{t \in\{0,1\}} \frac{a_{z, b}(t)}{p_{b}\left(1-p_{b}\right) m_{b} v_{z, b}(t)} \rightarrow 0
$$


(b) The correlation matrix of $\boldsymbol{t}$ has a limiting value $\boldsymbol{\Sigma}$.

(c) We have limiting values on the following variance expressions: $m \operatorname{Var}\left(\bar{w}_{b}^{1}\right)$ and $m \operatorname{Var}\left(\bar{u}_{b}(z)\right)$ for all $b \in\{1, \ldots, h\}$ and $z \in\{0,1\}$.

(d) $\bar{U}_{b}(1) \neq 0$ or $\bar{U}_{b}(0) \neq 0$ for some $b$.

Then we have

$$
\frac{\tilde{\beta}_{1}-\beta_{1}}{\sqrt{\operatorname{Var}\left(\tilde{\beta}_{1}\right)}} \stackrel{d}{\rightarrow} N(0,1)
$$

where

$$
\begin{aligned}
\operatorname{Var}\left(\tilde{\beta}_{1}\right)=\sum_{b=1}^{h} & \frac{1}{m_{b}\left(m_{b}-1\right)} \sum_{j=1}^{m_{b}}\left(\frac{\tilde{q}_{b} p_{b}\left(1-p_{b}\right)\left(1-2 p_{b}\right)}{\sqrt{p_{b}\left(1-p_{b}\right)}\left(\sum_{a=1}^{h} \tilde{q}_{a} p_{a}\left(1-p_{a}\right) \bar{w}_{a}\right)}\left(\beta_{1, b}-\beta_{1}\right)\left(w_{j b}-\bar{w}_{b}\right)\right. \\
& +\sqrt{\frac{1-p_{b}}{p_{b}}} \frac{\tilde{q}_{b} p_{b}\left(1-p_{b}\right) \bar{w}_{b}}{\sum_{a=1}^{h} \tilde{q}_{a} p_{a}\left(1-p_{a}\right) \bar{w}_{a}}\left(\frac{U_{j b}(1)}{\bar{w}_{b}}-\frac{w_{j b}}{\bar{w}_{b}} \frac{\bar{U}_{b}(1)}{\bar{w}_{b}}\right) \\
& \left.-\sqrt{\frac{p_{b}}{1-p_{b}}} \frac{\tilde{q}_{b} p_{b}\left(1-p_{b}\right) \bar{w}_{b}}{\sum_{a=1}^{h} \tilde{q}_{a} p_{a}\left(1-p_{a}\right) \bar{w}_{a}}\left(\frac{U_{j b}(0)}{\bar{w}_{b}}-\frac{w_{j b}}{\bar{w}_{b}} \frac{\bar{U}_{b}(0)}{\bar{w}_{b}}\right)\right)^{2} \\
=\sum_{b=1}^{h} & \frac{\tilde{q}_{b} p_{b}\left(1-p_{b}\right)\left(1-2 p_{b}\right)}{m_{b}\left(m_{b}-1\right)} \sum_{j=1}^{m_{b}}\left(\frac{\tilde{q}_{b}\left(1-p_{b}\right)}{\left.p_{a}\left(1-p_{a}\right) \bar{w}_{a}\right)}\left(\beta_{1, b}^{h}-\beta_{1}\right)\left(w_{j b}-\bar{w}_{b}\right)\right. \\
& +\sqrt{\frac{1-p_{b}}{p_{b}}} \frac{\tilde{q}_{b} p_{b}\left(1-p_{b}\right) \bar{w}_{b}}{\sum_{a=1}^{h} \tilde{q}_{a} p_{a}\left(1-p_{a}\right) \bar{w}_{a}}\left(\frac{w_{j b}\left(\bar{Y}_{j b}(1)-\overline{\boldsymbol{x}}_{j b} \gamma\right)}{\bar{w}_{b}}-\frac{w_{j b}\left(\overline{\bar{Y}}_{b}(1)-\overline{\overline{\boldsymbol{x}}}_{b} \gamma\right)}{\bar{w}_{b}}\right) \\
& \left.\quad \sqrt{\frac{p_{b}}{1-p_{b}}} \frac{\tilde{q}_{b} p_{b}\left(1-p_{b}\right) \bar{w}_{b}}{\sum_{a=1}^{h} \tilde{q}_{a} p_{a}\left(1-p_{a}\right) \bar{w}_{a}}\left(\frac{w_{j b}\left(\bar{Y}_{j b}(0)-\overline{\boldsymbol{x}}_{j b} \gamma\right)}{\bar{w}_{b}}-\frac{w_{j b}\left(\overline{\bar{Y}}_{b}(0)-\overline{\overline{\boldsymbol{x}}}_{b} \gamma\right)}{\bar{w}_{b}}\right)\right)^{2}
\end{aligned}
$$

Proof. By Theorem 4 of Li and Ding (2017), if as $m \rightarrow \infty$

$$
\max _{1 \leq b \leq h} \max _{z \in\{w, U\}} \max _{t \in\{0,1\}} \frac{a_{z, b}(t)}{p_{b}\left(1-p_{b}\right) m_{b} v_{z, b}(t)} \rightarrow 0
$$

and the correlation matrix of $\boldsymbol{t}$ has a limiting value $\boldsymbol{\Sigma}$, then

$$
\left(\frac{\bar{u}_{1}(1)-\bar{U}_{1}(1)}{\sqrt{\operatorname{Var}\left(\bar{u}_{1}(1)\right)}}, \frac{\bar{u}_{1}(0)-\bar{U}_{1}(0)}{\sqrt{\operatorname{Var}\left(\bar{u}_{1}(0)\right)}}, \frac{\bar{w}_{1}^{1}-\bar{w}_{1}}{\sqrt{\operatorname{Var}\left(\bar{w}_{1}^{1}\right)}}, \cdots, \frac{\bar{u}_{h}(0)-\bar{U}_{h}(0)}{\sqrt{\operatorname{Var}\left(\bar{u}_{h}(0)\right)}}, \frac{\bar{w}_{h}^{1}-\bar{w}_{h}}{\sqrt{\operatorname{Var}\left(\bar{w}_{h}^{1}\right)}}\right) \stackrel{d}{\rightarrow} N(\mathbf{0}, \mathbf{\Sigma}) .
$$

To use the delta method given in Pashley (2019), we also require that $\boldsymbol{t}-\boldsymbol{T} \stackrel{p}{\rightarrow} 0$ (i.e., $\bar{w}_{b}^{1}-\bar{w}_{b} \stackrel{p}{\rightarrow} 0$ and $\bar{u}_{b}(z)-\bar{U}_{b}(z) \stackrel{p}{\rightarrow} 0$ for all $b \in\{1, \ldots, h\}$ and $\left.z \in\{0,1\}\right)$. This is satisfied by our assumption on limiting values of the variances (this can be seen directly from our prior results or Markov's inequality). 
We can determine the variance and covariances by noting that blocks are independent of each other, but random variables within blocks are dependent. We have

$$
\begin{gathered}
\operatorname{Var}\left(\bar{u}_{b}(t)\right)=\left(\frac{1}{m_{b}^{t}}-\frac{1}{m_{b}}\right) v_{z, U}(t), \\
\operatorname{Var}\left(\bar{w}_{b}^{t}\right)=\left(\frac{1}{m_{b}^{t}}-\frac{1}{m_{b}}\right) v_{z, w}(t), \\
\operatorname{Cov}\left(\bar{u}_{b}(1), \bar{u}_{b}(0)\right)=-\frac{1}{m_{b}} \frac{1}{m_{b}-1} \sum_{j=1}^{m_{b}}\left(U_{j b}(1)-\bar{U}_{b}(1)\right)\left(U_{j b}(0)-\bar{U}_{b}(0)\right), \\
\operatorname{Cov}\left(\bar{u}_{b}(1), \bar{w}_{b}^{1}\right)=\left(\frac{1}{m_{b}^{1}}-\frac{1}{m_{b}}\right) \frac{1}{m_{b}-1} \sum_{j=1}^{m_{b}}\left(U_{j b}(1)-\bar{U}_{b}(1)\right)\left(w_{j b}-\bar{w}_{b}\right),
\end{gathered}
$$

and

$$
\operatorname{Cov}\left(\bar{u}_{b}(0), \bar{w}_{b}^{1}\right)=-\frac{1}{m_{b}} \frac{1}{m_{b}-1} \sum_{j=1}^{m_{b}}\left(U_{j b}(0)-\bar{U}_{b}(0)\right)\left(w_{j b}-\bar{w}_{b}\right) .
$$

Now $g(\cdot): \mathbb{R}^{3 h} \rightarrow \mathbb{R}$ takes our vector $\boldsymbol{t}$ and returns the estimator $\tilde{\beta}_{1}$. As our weights are all positive and non-zero, this function is continuous and differential on the domain of $\boldsymbol{T}$. We have

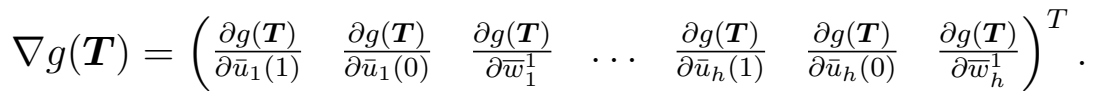

The partial derivatives are

$$
\begin{aligned}
\left.\frac{\partial \hat{\beta}}{\partial \bar{u}_{1}(1)}\right|_{\boldsymbol{T}}= & \frac{\tilde{q}_{b} p_{b}\left(1-p_{b}\right) \bar{w}_{b}}{\sum_{a=1}^{h} \tilde{q}_{a} p_{a}\left(1-p_{a}\right) \bar{w}_{a}} \frac{1}{\bar{w}_{b}} \\
\left.\frac{\partial \hat{\beta}}{\partial \bar{u}_{1}(0)}\right|_{\boldsymbol{T}}= & -\frac{\tilde{q}_{b} p_{b}\left(1-p_{b}\right) \bar{w}_{b}}{\sum_{a=1}^{h} \tilde{q}_{a} p_{a}\left(1-p_{a}\right) \bar{w}_{a}} \frac{1}{\bar{w}_{b}} \\
\left.\frac{\partial \hat{\beta}}{\partial \bar{w}_{b}}\right|_{\boldsymbol{T}}= & -\tilde{q}_{b} p_{b}\left(1-2 p_{b}\right) \sum_{c=1}^{h} \frac{\tilde{q}_{c} p_{c}\left(1-p_{c}\right) \bar{w}_{c}}{\left(\sum_{a=1}^{h} \tilde{q}_{a} p_{a}\left(1-p_{a}\right) \bar{w}_{a}\right)^{2}}\left(\frac{\bar{U}_{c}(1)}{\bar{w}_{c}}-\frac{\bar{U}_{c}(0)}{\bar{w}_{c}}\right) \\
& -\frac{\tilde{q}_{b} p_{b}\left(1-p_{b}\right)}{\sum_{a=1}^{h} \tilde{q}_{a} p_{a}\left(1-p_{a}\right) \bar{w}_{a}}\left(\frac{p_{b}}{1-p_{b}} \frac{\bar{U}_{b}(1)}{\bar{w}_{b}}+\frac{\bar{U}_{b}(0)}{\bar{w}_{b}}\right) \\
= & -\frac{\tilde{q}_{b} p_{b}\left(1-2 p_{b}\right)}{\sum_{a=1}^{h} \tilde{q}_{a} p_{a}\left(1-p_{a}\right) \bar{w}_{a}} \beta_{1}-\frac{\tilde{q}_{b} p_{b}\left(1-p_{b}\right) \bar{w}_{b}}{\sum_{a=1}^{h} \tilde{q}_{a} p_{a}\left(1-p_{a}\right) \bar{w}_{a}}\left(\frac{p_{b}}{1-p_{b}} \frac{\bar{U}_{b}(1)}{\left(\bar{w}_{b}\right)^{2}}+\frac{\bar{U}_{b}(0)}{\left(\bar{w}_{b}\right)^{2}}\right) .
\end{aligned}
$$

We see that all of these derivatives are continuous and, assuming that we do not have $\bar{U}_{b}(0)=\bar{U}_{b}(0)=0$ for all $b$, we can therefore use the delta method result of Theorem 2 of Pashley (2019), which gives

$$
\frac{g(\boldsymbol{t})-g(\boldsymbol{T})}{\sqrt{(\nabla g(\boldsymbol{T}))^{T} \boldsymbol{V} \boldsymbol{\Sigma} \boldsymbol{V} \nabla g(\boldsymbol{T})}} \stackrel{d}{\rightarrow} \mathrm{N}(0,1)
$$


where

$$
\boldsymbol{V}=\left(\begin{array}{cccc}
\sqrt{\operatorname{Var}\left(\bar{u}_{1}(1)\right)} & 0 & \cdots & 0 \\
0 & \sqrt{\operatorname{Var}\left(\bar{u}_{1}(0)\right)} & \cdots & 0 \\
\vdots & \vdots & \ddots & \vdots \\
0 & 0 & \cdots & \sqrt{\operatorname{Var}\left(\bar{w}_{h}^{1}\right)}
\end{array}\right)
$$

The denominator corresponds to the variance of $\tilde{\beta}_{1}$. Let $\beta_{1, b}=\left(\bar{U}_{b}(1)-\bar{U}_{b}(0)\right) / \bar{w}_{b}=$ $\overline{\bar{Y}}_{b}(1)-\overline{\bar{Y}}_{b}(0)$. We can now do the multiplication to get the variance, noting that symmetry can greatly simplify the calculations. Replacing the limiting values of $\boldsymbol{\Sigma}$ with the sample values, we get

$$
\begin{aligned}
\operatorname{Var}\left(\tilde{\beta}_{1}\right)=\sum_{b=1}^{h} & \frac{1}{m_{b}\left(m_{b}-1\right)} \sum_{j=1}^{m_{b}}\left(-\frac{\sqrt{1-p_{b}} \tilde{q}_{b} p_{b}\left(1-2 p_{b}\right)}{\sqrt{p_{b}}\left(\sum_{a=1}^{h} \tilde{q}_{a} p_{a}\left(1-p_{a}\right) \bar{w}_{a}\right)} \beta_{1}\left(w_{j b}-\bar{w}_{b}\right)\right. \\
& -\sqrt{\frac{1-p_{b}}{p_{b}}} \frac{\tilde{q}_{b} p_{b}\left(1-p_{b}\right) \bar{w}_{b}}{\sum_{a=1}^{h} \tilde{q}_{a} p_{a}\left(1-p_{a}\right) \bar{w}_{a}}\left(\frac{p_{b}}{1-p_{b}} \frac{\bar{U}_{b}(1)}{\left(\bar{w}_{b}\right)^{2}}+\frac{\left.\bar{U}_{b}(0)\right)}{\left(\bar{w}_{b}\right)^{2}}\right)\left(w_{j b}-\bar{w}_{b}\right) \\
& +\sqrt{\frac{1-p_{b}}{p_{b}}} \frac{\tilde{q}_{b} p_{b}\left(1-p_{b}\right) \bar{w}_{b}}{\sum_{a=1}^{h} \tilde{q}_{a} p_{a}\left(1-p_{a}\right) \bar{w}_{a}}\left(\frac{U_{j b}(1)}{\bar{w}_{b}}-\frac{\bar{U}_{b}(1)}{\bar{w}_{b}}\right) \\
& \left.+\sqrt{\frac{p_{b}}{1-p_{b}}} \frac{\tilde{q}_{b} p_{b}\left(1-p_{b}\right) \bar{w}_{b}}{\sum_{a=1}^{h} \tilde{q}_{a} p_{a}\left(1-p_{a}\right) \bar{w}_{a}}\left(\frac{U_{j b}(0)}{\bar{w}_{b}}-\frac{\bar{U}_{b}(0)}{\bar{w}_{b}}\right)\right)^{2} .
\end{aligned}
$$

We can rewrite this more digestibly in terms of block estimators as

$$
\begin{aligned}
\operatorname{Var}\left(\tilde{\beta}_{1}\right)=\sum_{b=1}^{h} & \frac{1}{m_{b}\left(m_{b}-1\right)} \sum_{j=1}^{m_{b}}\left(\frac{\tilde{q}_{b} p_{b}\left(1-p_{b}\right)\left(1-2 p_{b}\right)}{\sqrt{p_{b}\left(1-p_{b}\right)}\left(\sum_{a=1}^{h} \tilde{q}_{a} p_{a}\left(1-p_{a}\right) \bar{w}_{a}\right)}\left(\beta_{1, b}-\beta_{1}\right)\left(w_{j b}-\bar{w}_{b}\right)\right. \\
& +\sqrt{\frac{1-p_{b}}{p_{b}}} \frac{\tilde{q}_{b} p_{b}\left(1-p_{b}\right) \bar{w}_{b}}{\sum_{a=1}^{h} \tilde{q}_{a} p_{a}\left(1-p_{a}\right) \bar{w}_{a}}\left(\frac{w_{j b}\left(\bar{Y}_{j b}(1)-\overline{\boldsymbol{x}}_{j b} \gamma\right)}{\bar{w}_{b}}-\frac{w_{j b}\left(\overline{\bar{Y}}_{b}(1)-\overline{\boldsymbol{x}}_{b} \gamma\right)}{\bar{w}_{b}}\right) \\
& \left.+\sqrt{\frac{p_{b}}{1-p_{b}}} \frac{\tilde{q}_{b} p_{b}\left(1-p_{b}\right) \bar{w}_{b}}{\sum_{a=1}^{h} \tilde{q}_{a} p_{a}\left(1-p_{a}\right) \bar{w}_{a}}\left(\frac{w_{j b}\left(\bar{Y}_{j b}(0)-\overline{\boldsymbol{x}}_{j b} \gamma\right)}{\bar{w}_{b}}-\frac{w_{j b}\left(\overline{\bar{Y}}_{b}(0)-\overline{\bar{x}}_{b} \gamma\right)}{\bar{w}_{b}}\right)\right)^{2} .
\end{aligned}
$$

\section{A.4.4 Theorem 2: Asymptotic normality with estimated $\hat{\gamma}$}

Finally, to prove Theorem 2 we can follow the same proof as in Section A.3.5.

Theorem 2 assume the conditions of Theorem A.1, the conditions of Lemma A.1.1 applied to each of the components of $\overline{\overline{\boldsymbol{x}}}_{b}^{1}$ and $\overline{\boldsymbol{x}}_{b}^{0}$, and a limiting value on the asymptotic variance for 
those components. It also assumes limiting values on the following variance expressions:

$$
\begin{aligned}
\boldsymbol{S}_{\boldsymbol{x}, b}^{2} & =\frac{1}{m_{b}} \sum_{j=1}^{m_{b}} \sum_{i=1}^{n_{j b}} w_{i j b}\left(\boldsymbol{x}_{i j b}-\overline{\overline{\boldsymbol{x}}}_{b}\right)^{\prime}\left(\boldsymbol{x}_{i j b}-\overline{\overline{\boldsymbol{x}}}_{b}\right) \\
\boldsymbol{S}_{\boldsymbol{x}, Y, b}^{2}(t) & =\frac{1}{m_{b}} \sum_{j=1}^{m_{b}} \sum_{i=1}^{n_{j b}} w_{i j b} \boldsymbol{x}_{i j b}^{\prime} Y_{i j b}(t)-\overline{\overline{\boldsymbol{x}}}_{b}^{\prime} \overline{w Y(t)_{b}} \\
\boldsymbol{S}_{\boldsymbol{x} Y, b}^{2}(t) & =\frac{1}{m_{b}} \sum_{j=1}^{m_{b}} \sum_{i=1}^{n_{j b}}\left(w_{i j b} \boldsymbol{x}_{i j b}^{\prime} Y_{i j b}(t)-\overline{w x Y(t)_{b}}\right)^{2} \text { for } t \in\{0,1\} \text { with } \\
\overline{w x Y(t)_{b}} & =\frac{1}{m_{b}} \sum_{j=1}^{m_{b}} \sum_{i=1}^{n_{j b}} w_{i j b} \boldsymbol{x}_{i j b}^{\prime} Y_{i j b}(t) .
\end{aligned}
$$

Then, we have the result of Theorem 2,

$$
\frac{\hat{\beta}_{1}-\beta_{1}}{\sqrt{\operatorname{Var}\left(\tilde{\beta}_{1}\right)}} \stackrel{d}{\rightarrow} N(0,1) .
$$

with

$$
\begin{aligned}
& \operatorname{Var}\left(\hat{\beta}_{1}\right)=\sum_{b=1}^{h} \frac{1}{m_{b}\left(m_{b}-1\right)} \sum_{j=1}^{m_{b}}\left(\frac{\tilde{q}_{b} p_{b}\left(1-p_{b}\right)\left(1-2 p_{b}\right)}{\sqrt{p_{b}\left(1-p_{b}\right)}\left(\sum_{a=1}^{h} \tilde{q}_{a} p_{a}\left(1-p_{a}\right) \bar{w}_{a}\right)}\left(\beta_{1, b}-\beta_{1}\right)\left(w_{j b}-\bar{w}_{b}\right)\right. \\
& +\sqrt{\frac{1-p_{b}}{p_{b}}} \frac{\tilde{q}_{b} p_{b}\left(1-p_{b}\right) \bar{w}_{b}}{\sum_{a=1}^{h} \tilde{q}_{a} p_{a}\left(1-p_{a}\right) \bar{w}_{a}}\left(\frac{U_{j b}(1)}{\bar{w}_{b}}-\frac{w_{j b}}{\bar{w}_{b}} \frac{\bar{U}_{b}(1)}{\bar{w}_{b}}\right) \\
& \left.+\sqrt{\frac{p_{b}}{1-p_{b}}} \frac{\tilde{q}_{b} p_{b}\left(1-p_{b}\right) \bar{w}_{b}}{\sum_{a=1}^{h} \tilde{q}_{a} p_{a}\left(1-p_{a}\right) \bar{w}_{a}}\left(\frac{U_{j b}(0)}{\bar{w}_{b}}-\frac{w_{j b}}{\bar{w}_{b}} \frac{\bar{U}_{b}(0)}{\bar{w}_{b}}\right)\right)^{2} \\
& =\sum_{b=1}^{h} \frac{1}{m_{b}\left(m_{b}-1\right)} \sum_{j=1}^{m_{b}}\left(\frac{\tilde{q}_{b} p_{b}\left(1-p_{b}\right)\left(1-2 p_{b}\right)}{\sqrt{p_{b}\left(1-p_{b}\right)}\left(\sum_{a=1}^{h} \tilde{q}_{a} p_{a}\left(1-p_{a}\right) \bar{w}_{a}\right)}\left(\beta_{1, b}-\beta_{1}\right)\left(w_{j b}-\bar{w}_{b}\right)\right. \\
& +\sqrt{\frac{1-p_{b}}{p_{b}}} \frac{\tilde{q}_{b} p_{b}\left(1-p_{b}\right) \bar{w}_{b}}{\sum_{a=1}^{h} \tilde{q}_{a} p_{a}\left(1-p_{a}\right) \bar{w}_{a}}\left(\frac{w_{j b}\left(\bar{Y}_{j b}(1)-\overline{\boldsymbol{x}}_{j b} \gamma\right)}{\bar{w}_{b}}-\frac{w_{j b}\left(\overline{\bar{Y}}_{b}(1)-\overline{\overline{\boldsymbol{x}}}_{b} \gamma\right)}{\bar{w}_{b}}\right) \\
& \left.+\sqrt{\frac{p_{b}}{1-p_{b}}} \frac{\tilde{q}_{b} p_{b}\left(1-p_{b}\right) \bar{w}_{b}}{\sum_{a=1}^{h} \tilde{q}_{a} p_{a}\left(1-p_{a}\right) \bar{w}_{a}}\left(\frac{w_{j b}\left(\bar{Y}_{j b}(0)-\overline{\boldsymbol{x}}_{j b} \gamma\right)}{\bar{w}_{b}}-\frac{w_{j b}\left(\overline{\bar{Y}}_{b}(0)-\overline{\overline{\boldsymbol{x}}}_{b} \gamma\right)}{\bar{w}_{b}}\right)\right)^{2} .
\end{aligned}
$$

\section{A.5 Asymptotic Efficiency Losses Using the Individual Data}

Corollary 1 to Theorem 1 in the main text shows that aggregating (averaging) the data to the cluster level produces asymptotically efficient ATE estimators. To quantify efficiency losses using the individual data, we first express the $\gamma$ parameter as a weighted 
average of between- and within-cluster population regression parameters, $\gamma_{\boldsymbol{B}}$ and $\gamma_{\boldsymbol{W}}$, using $\boldsymbol{\gamma}=\boldsymbol{\Gamma}_{\boldsymbol{x}} \boldsymbol{\gamma}_{B}+\left(\boldsymbol{I}_{v \times v}-\boldsymbol{\Gamma}_{\boldsymbol{x}}\right) \boldsymbol{\gamma}_{\boldsymbol{W}}$. In this expression, $\boldsymbol{\gamma}_{\boldsymbol{B}}$ is defined analogously to $\boldsymbol{\gamma}$ in Section A.3.3 in the supplementary materials (and in Remark 2 of the main text) by replacing $\boldsymbol{S}_{\boldsymbol{x}, b}^{2}$ with $\boldsymbol{S}_{\boldsymbol{x}, b, \boldsymbol{B}}^{2}=\frac{1}{m_{b}} \sum_{j=1}^{m_{b}} w_{j b}\left(\overline{\boldsymbol{x}}_{j b}-\overline{\overline{\boldsymbol{x}}}_{b}\right)^{\prime}\left(\overline{\boldsymbol{x}}_{j b}-\overline{\overline{\boldsymbol{x}}}_{b}\right)$, and using parallel clusterlevel versions of $\boldsymbol{S}_{\boldsymbol{x}, Y, b}^{2}(1)$ and $\boldsymbol{S}_{\boldsymbol{x}, Y, b}^{2}(0)$. This is the regression parameter that would be obtained using the full schedule of potential outcomes and covariates aggregated to the cluster level. We define $\gamma_{\boldsymbol{W}}$ similarly, for example, by replacing $\boldsymbol{S}_{\boldsymbol{x}, b}^{2}$ with $\boldsymbol{S}_{\boldsymbol{x}, b, \boldsymbol{W}}^{2}=$ $\frac{1}{m_{b}} \sum_{j=1}^{m_{b}} \sum_{i=1}^{n_{j b}} w_{i j b}\left(\boldsymbol{x}_{i j b}-\overline{\boldsymbol{x}}_{j b}\right)^{\prime}\left(\boldsymbol{x}_{i j b}-\overline{\boldsymbol{x}}_{j b}\right)$. The intraclass correlation coefficient (ICC) matrix, $\boldsymbol{\Gamma}_{\boldsymbol{x}}$, is defined as $\boldsymbol{\Gamma}_{\boldsymbol{x}}=\left(\boldsymbol{S}_{\boldsymbol{x}, \boldsymbol{B}}^{2}+\boldsymbol{S}_{\boldsymbol{x}, \boldsymbol{W}}^{2}\right)^{-1} \boldsymbol{S}_{\boldsymbol{x}, \boldsymbol{B}}^{2}$, where $\boldsymbol{S}_{\boldsymbol{x}, \boldsymbol{B}}^{2}=\sum_{b=1}^{h} q_{b} \boldsymbol{S}_{\boldsymbol{x}, b, \boldsymbol{B}}^{2}, \boldsymbol{S}_{\boldsymbol{x}, \boldsymbol{W}}^{2}=$ $\sum_{b=1}^{h} q_{b} \boldsymbol{S}_{\boldsymbol{x}, b, \boldsymbol{W}}^{2}$, and $\boldsymbol{I}_{v \times v}$ is the identity matrix. This ICC matrix measures the proportions of the total variances and covariances of the covariates that are due to between-cluster variation.

Next note that $S_{D_{b}}^{2}(1)$ and $S_{D_{b}}^{2}(0)$ in (8) in the main text are based on cluster-level residuals. Thus, the within-cluster covariates, $\left(\boldsymbol{x}_{i j}-\overline{\boldsymbol{x}}_{j}\right)$, have no effect on $S_{D_{b}}^{2}(t)$, and hence, on precision. Accordingly, $\gamma$ as defined using the individual data is inefficient. Intuitively, the within-cluster covariates "contaminate" $\boldsymbol{\gamma}$. Comparing $\gamma$ to the optimal $\gamma_{B}$, the difference is $\left(\boldsymbol{I}_{v \times v}-\boldsymbol{\Gamma}_{\boldsymbol{x}}\right)\left(\boldsymbol{\gamma}_{\boldsymbol{W}}-\boldsymbol{\gamma}_{\boldsymbol{B}}\right)$. Thus, asymptotic efficiency losses using the individual data will depend on $\boldsymbol{\Gamma}_{\boldsymbol{x}}$ and the relative values of $\boldsymbol{\gamma}_{\boldsymbol{B}}$ and $\boldsymbol{\gamma}_{\boldsymbol{W}}$. For instance, if the model only contains cluster-level covariates, then $\boldsymbol{\Gamma}_{\boldsymbol{x}}=\boldsymbol{I}_{v \times v}$ and there are no efficiency losses. However, if the covariates vary both within and between clusters, $\gamma$ is inefficient.

\section{A.6 TX collinearity $\left(R^{2}\right)$ results using the individual and aggre- gate data}

We next argue for the following two-part result:

\section{Result:}

Consider a non-blocked, clustered RCT design (where we consider a non-blocked design to reduce notation). Further let the covariate matrix, $\boldsymbol{X}$, have full rank. Then we have:

1. For any treatment allocation, the $R_{T X B}^{2}$ value from a WLS regression of $\widetilde{T}_{j}$ on $\widetilde{\overline{\boldsymbol{x}}}_{j}=$ $\left(\overline{\boldsymbol{x}}_{j}-\overline{\overline{\boldsymbol{x}}}\right)$ using the aggregate data will be at least as large as the $R_{T X}^{2}$ value from a WLS regression of $\widetilde{T}_{j}$ on $\widetilde{\boldsymbol{x}}_{i j}$ using the individual data.

2. If we assume equal cluster sizes $\left(\frac{n}{m}\right)$ and weights of 1 , we can approximate $E\left(R_{T X B}^{2}\right)$ using $\frac{v}{m}$ and $E\left(R_{T X}^{2}\right)$ using $\frac{\operatorname{tr}\left(\boldsymbol{\Gamma}_{\boldsymbol{x}}\right)}{m}+\frac{\left(v-\operatorname{tr}\left(\boldsymbol{\Gamma}_{\boldsymbol{x}}\right)\right)}{n}$, where $t r$ is the trace operator and $\boldsymbol{\Gamma}_{\boldsymbol{x}}$ is a $v \times v$ matrix of intraclass correlation coefficients (ICCs) for the covariates (defined below). Here, the expectation is taken over the randomization distribution.

Proof. To establish that the aggregate $R_{T X B}^{2}$ is is larger than the individual $R_{T X}^{2}$ for a given treatment allocation, we first define $\widehat{\boldsymbol{\lambda}}_{B}$ to be the estimated parameter from a WLS regression of $\widetilde{T}_{j}$ on $\widetilde{\overline{\boldsymbol{x}}}_{j}=\left(\overline{\boldsymbol{x}}_{j}-\overline{\overline{\boldsymbol{x}}}\right)$ using the aggregate data. We have that $\widehat{\boldsymbol{\lambda}}_{B}=\left(\widetilde{\overline{\boldsymbol{X}}}^{\prime} \overline{\boldsymbol{W}} \widetilde{\overline{\boldsymbol{X}}}^{-1} \widetilde{\widetilde{\boldsymbol{X}}}^{\prime} \overline{\boldsymbol{W}} \widetilde{\boldsymbol{T}}\right.$, where $\widetilde{\boldsymbol{X}}$ is an $m \times v$ matrix of $\widetilde{\overline{\boldsymbol{x}}}_{j}$ values, $\overline{\boldsymbol{W}}$ is an $m \times m$ diagonal matrix of $w_{j}$ weights, and $\widetilde{\boldsymbol{T}}$ is a vector of $\widetilde{T}_{j}=T_{j}-p^{*}$ values, with $p^{*}=\frac{1}{\sum_{j=1}^{m} w_{j}} \sum_{j=1}^{m} T_{j} w_{j}$. Next, using the 
individual data, consider a WLS regression of $\widetilde{T}_{j}$ on between- and within-cluster covariates, $\left(\overline{\boldsymbol{x}}_{j}-\overline{\overline{\boldsymbol{x}}}\right)$ and $\left(\boldsymbol{x}_{i j}-\overline{\boldsymbol{x}}_{j}\right)$, with associated parameter vectors, $\boldsymbol{\pi}_{B}$ and $\boldsymbol{\pi}_{W}$. We have $\widehat{\boldsymbol{\pi}}_{W}=\mathbf{0}$ because treatment assignments are at the cluster level, and thus, $\widehat{\boldsymbol{\pi}}_{B}=\widehat{\boldsymbol{\lambda}}_{B}$.

If we now instead use the individual data to regress $\widetilde{T}_{j}$ on a single set of covariates, $\widetilde{\boldsymbol{x}}_{i j}$, with associated parameter vector, $\boldsymbol{\pi}$, standard decomposition results (see, e.g., Greene, 2018) establish that $\widehat{\boldsymbol{\pi}}=\boldsymbol{\Gamma}_{\boldsymbol{x}} \widehat{\boldsymbol{\lambda}}_{B}+\left(\boldsymbol{I}_{v \times v}-\boldsymbol{\Gamma}_{\boldsymbol{x}}\right) \widehat{\boldsymbol{\lambda}}_{W}=\boldsymbol{\Gamma}_{\boldsymbol{x}} \widehat{\boldsymbol{\lambda}}_{B}$, where $\boldsymbol{\Gamma}_{\boldsymbol{x}}=\left(\widetilde{\boldsymbol{X}}^{\prime} \boldsymbol{W} \widetilde{\boldsymbol{X}}\right)^{-1}\left(\widetilde{\overline{\boldsymbol{X}}}^{\prime} \overline{\boldsymbol{W}} \widetilde{\overline{\boldsymbol{X}}}\right)$ is a $v \times v$ matrix of ICCs for the covariates (that measures the proportions of the total variances and covariances of the covariates that are between clusters), $\widetilde{\boldsymbol{X}}$ is an $n \times v$ matrix of $\widetilde{\boldsymbol{x}}_{j}$ values, $\boldsymbol{W}$ is an $n \times n$ diagonal matrix of $w_{i j}$ weights, and $\boldsymbol{I}_{v \times v}$ is the identity matrix.

Using these results and noting that the regression $R^{2}$ value is the explained sum of squares divided by the total sum of squares, we have that $R_{T X B}^{2}=\frac{1}{n p^{*}\left(1-p^{*}\right)}\left(\widetilde{\boldsymbol{T}}^{\prime} \overline{\boldsymbol{W}} \widetilde{\overline{\boldsymbol{X}}}\right)\left(\widetilde{\widetilde{\boldsymbol{X}}}^{\prime} \overline{\boldsymbol{W}} \widetilde{\overline{\boldsymbol{X}}}^{-1}\left(\widetilde{\overline{\boldsymbol{X}}}^{\prime} \overline{\boldsymbol{W}} \widetilde{\boldsymbol{T}}\right)\right.$, and similarly for $R_{T X}^{2}$, which yields

$$
R_{T X B}^{2}-R_{T X}^{2}=\frac{1}{n p^{*}\left(1-p^{*}\right)}\left(\widetilde{\boldsymbol{T}}^{\prime} \overline{\boldsymbol{W}} \widetilde{\overline{\boldsymbol{X}}}\right)\left(\boldsymbol{I}_{v \times v}-\boldsymbol{\Gamma}_{\boldsymbol{x}}\right)\left({\widetilde{\overline{\boldsymbol{X}}^{\prime}}}^{\prime} \overline{\boldsymbol{W}}_{\overline{\boldsymbol{X}}}\right)^{-1}\left(\widetilde{\overline{\boldsymbol{X}}}^{\prime} \overline{\boldsymbol{W}} \widetilde{\boldsymbol{T}}\right) .
$$

This result establishes that $\left(R_{T X B}^{2}-R_{T X}^{2}\right) \geq 0$ because $\left(\boldsymbol{I}_{v \times v}-\boldsymbol{\Gamma}_{\boldsymbol{x}}\right)\left(\widetilde{\boldsymbol{X}}^{\prime} \overline{\boldsymbol{W}} \widetilde{\overline{\boldsymbol{X}}}\right)^{-1}$ is positive semi-definite and $\left(\widetilde{\boldsymbol{T}}^{\prime} \overline{\boldsymbol{W}} \widetilde{\overline{\boldsymbol{X}}}\right)$ has full rank, with equality if and only if the covariates do not vary within clusters (in which case $\boldsymbol{\Gamma}_{\boldsymbol{x}}=\boldsymbol{I}_{v \times v}$ ).

To next establish the approximation results for $E\left(R_{T X B}^{2}\right)$ and $E\left(R_{T X}^{2}\right)$, we assume equal cluster sizes $\left(\frac{n}{m}\right)$ and weights of 1 . In this case, for the aggregate data, we have that $E\left(R_{T X B}^{2}\right)=1-\frac{1}{m p(1-p)} E\left(\widetilde{\boldsymbol{T}}^{\prime}\left(\boldsymbol{I}-\boldsymbol{P}_{\widetilde{\boldsymbol{X}}}\right) \widetilde{\boldsymbol{T}}\right)$, where $\boldsymbol{P}_{\widetilde{\boldsymbol{X}}}=\widetilde{\overline{\boldsymbol{X}}}^{\prime}\left(\widetilde{\widetilde{\boldsymbol{X}}}^{\prime} \widetilde{\overline{\boldsymbol{X}}}\right)^{-1} \widetilde{\overline{\boldsymbol{X}}}^{\prime}$ is the projection matrix. Using the trace operator, we have that $E\left(R_{T X B}^{2}\right)=1-\frac{1}{m p(1-p)} \operatorname{tr}\left[\left(\boldsymbol{I}-\boldsymbol{P}_{\widetilde{\boldsymbol{X}}}\right) \boldsymbol{\Lambda}\right]$, where $\boldsymbol{\Lambda}=E\left(\widetilde{\boldsymbol{T}} \widetilde{\boldsymbol{T}}^{\prime}\right)$ has diagonal elements, $p(1-p)$, and off-diagonal covariances, $-\frac{p(1-p)}{(m-1)}$. If we ignore the small covariances in $\Lambda$, we find that $E\left(R_{T X B}^{2}\right) \approx \frac{v}{m}$. Similarly, for the individual data, we have that $E\left(R_{T X}^{2}\right)=1-\frac{1}{m p(1-p)} E\left(\widetilde{\boldsymbol{T}}^{\prime}\left(\boldsymbol{I}-\boldsymbol{P}_{\widetilde{\boldsymbol{X}}}\right) \widetilde{\boldsymbol{T}}\right)$, where $\boldsymbol{P}_{\widetilde{\boldsymbol{X}}}=$ $\widetilde{\boldsymbol{X}}^{\prime} \Gamma_{\boldsymbol{x}}\left(\widetilde{\boldsymbol{X}}^{\prime} \widetilde{\boldsymbol{X}}\right)^{-1} \widetilde{\boldsymbol{X}}^{\prime}$ is the new projection matrix. If we again use the trace operator and ignore the off-diagonal elements in $\boldsymbol{\Lambda}$, we find that $E\left(R_{T X}^{2}\right) \approx \frac{\operatorname{tr}\left(\boldsymbol{\Gamma}_{\boldsymbol{x}}\right)}{m}$. To avoid a zero expected value when $\operatorname{tr}\left(\boldsymbol{\Gamma}_{\boldsymbol{x}}\right)=0$, we instead use an alternative approximation, $E\left(R_{T X}^{2}\right) \approx \frac{\operatorname{tr}\left(\boldsymbol{\Gamma}_{\boldsymbol{x}}\right)}{m}+\frac{\left(v-\operatorname{tr}\left(\boldsymbol{\Gamma}_{\boldsymbol{x}}\right)\right)}{n}$.

To motivate this approximation for $E\left(R_{T X}^{2}\right)$, we express it as $E\left(R_{T X}^{2}\right) \approx \frac{v}{n^{*}}$, where $n^{*}=\frac{n}{1+\bar{\rho}_{X}(\bar{n}-1)}$ is the "effective" sample size of individuals, $\bar{\rho}_{X}=\frac{\operatorname{tr}\left(\boldsymbol{\Gamma}_{\boldsymbol{x}}\right)}{v}$ is the average covariate ICC for the variances, and $\bar{n}=\frac{n}{m}$ is the average cluster size. The denominator term, $\left[1+\bar{\rho}_{X}(\bar{n}-1)\right]$, can be considered a design effect due to covariate clustering. As $\bar{\rho}_{X}$ approaches $0, n^{*}$ approaches $n$ (minimum design effects), whereas as $\bar{\rho}_{X}$ approaches $1, n^{*}$ approaches $m$ (maximum design effects).

Table A.1 displays simulation results that support the use of these approximations. For the simulations, we randomly generated $v=2,5$, or 10 covariates using $x_{i j 1}=u_{j 1}+e_{i j 1}$ and $x_{i j k}=\theta x_{i j(k-1)}+u_{j k}+e_{i j k}$ for $k=2, \ldots, v$, where $u_{j q}$ and $e_{j q}$ are independently and identically 
distributed normal random errors with mean zero. The within-cluster errors, $e_{j q}$, were set to have variance 1 , and the variances of the between-cluster errors, $u_{j q}$, were calculated based on assumed ICC values of $\rho_{X}=0,0.4$, or 0.8 . The parameter, $\theta$, was calculated to generate a 0.5 correlation coefficient $(r)$ between $x_{i j k}$ and $x_{i j(k-1)}$. Specifically, we set $\theta=\frac{r}{\sqrt{1-r^{2}}}=.577$. The number of clusters ranged from $m=20$ to 60 , and to allow for some unbalance in the design, we set $p=.6$ and allowed the number of individuals per cluster to range uniformly between $n_{j}=25$ and 75 . For each model specification, we generated a single dataset of covariates and then generated 500 draws from the randomization distribution. We repeated this procedure 10 times to avoid unusual base datasets and calculated average results. Table A.1 presents mean $R_{T X B}^{2}$ and $R_{T X}^{2}$ values across the 5,000 simulations using the individual and aggregate data (the "true" values) as well as the $E\left(R_{T X B}^{2}\right)$ and $E\left(R_{T X}^{2}\right)$ approximations from above.

The results indicate that the $E\left(R_{T X B}^{2}\right)$ approximations using the aggregate data are close to true values, with slight downward biases as $\rho_{X}$ values increase. The $E\left(R_{T X}^{2}\right)$ approximations are also reasonably close to true values, with no patterns of downward or upward biases. 
Table A.1. Simulation results for the $E\left(R_{T X B}^{2}\right)$ and $E\left(R_{T X}^{2}\right)$ approximations

\begin{tabular}{|c|c|c|c|c|c|c|c|}
\hline \multirow[b]{2}{*}{$\begin{array}{l}\text { Number } \\
\text { of } \\
\text { clusters }\end{array}$} & \multirow[b]{2}{*}{$\begin{array}{c}\text { ICC of } \\
\text { covariates } \\
\left(\rho_{X}\right)\end{array}$} & \multicolumn{3}{|c|}{ Aggregate data } & \multicolumn{3}{|c|}{ Individual data } \\
\hline & & $\begin{array}{c}\text { Average } R_{T X B}^{2} \\
\text { value across } \\
\text { simulations }\end{array}$ & $\begin{array}{l}\text { Approximation } \\
\text { for } E\left(R_{T X B}^{2}\right)\end{array}$ & Ratio & $\begin{array}{c}\text { Average } R_{T X}^{2} \\
\text { value across } \\
\text { simulations }\end{array}$ & $\begin{array}{l}\text { Approximation } \\
\text { for } E\left(R_{T X}^{2}\right)\end{array}$ & Ratio \\
\hline \multicolumn{8}{|c|}{$v=2$ covariates } \\
\hline 20 & 0 & .101 & .100 & 1.01 & .002 & .002 & 1.00 \\
\hline 20 & .4 & .111 & .100 & 1.11 & .043 & .041 & 1.05 \\
\hline 20 & .8 & .113 & .100 & 1.13 & .123 & .080 & 1.54 \\
\hline 40 & 0 & .052 & .050 & 1.04 & .001 & .001 & 1.00 \\
\hline 40 & .4 & .055 & .050 & 1.10 & .027 & .021 & 1.29 \\
\hline 40 & .8 & .056 & .050 & 1.12 & .031 & .040 & 0.78 \\
\hline 60 & 0 & .033 & .033 & 1.00 & .001 & .001 & 1.00 \\
\hline 60 & .4 & .036 & .033 & 1.09 & .008 & .014 & 0.57 \\
\hline 60 & .8 & .037 & .033 & 1.12 & .036 & .027 & 1.33 \\
\hline \multicolumn{8}{|c|}{$v=5$ covariates } \\
\hline 20 & 0 & .263 & .250 & 1.05 & .007 & .005 & 1.40 \\
\hline 20 & .4 & .272 & .250 & 1.09 & .107 & .103 & 1.04 \\
\hline 20 & .8 & .274 & .250 & 1.10 & .207 & .201 & 1.03 \\
\hline 40 & 0 & .129 & .125 & 1.03 & .001 & .003 & 0.33 \\
\hline 40 & .4 & .137 & .125 & 1.10 & .041 & .052 & 0.79 \\
\hline 40 & .8 & .136 & .125 & 1.09 & .112 & .101 & 1.11 \\
\hline 60 & 0 & .084 & .083 & 1.01 & .002 & .002 & 1.00 \\
\hline 60 & .4 & .090 & .083 & 1.08 & .041 & .034 & 1.21 \\
\hline 60 & .8 & .092 & .083 & 1.11 & .081 & .067 & 1.21 \\
\hline \multicolumn{8}{|c|}{$v=10$ covariates } \\
\hline 20 & 0 & .526 & .500 & 1.05 & .010 & .010 & 1.00 \\
\hline 20 & .4 & .548 & .500 & 1.10 & .179 & .206 & 0.87 \\
\hline 20 & .8 & .551 & .500 & 1.10 & .331 & .402 & 0.82 \\
\hline 40 & 0 & .256 & .250 & 1.02 & .005 & .005 & 1.00 \\
\hline 40 & .4 & .270 & .250 & 1.08 & .091 & .103 & 0.88 \\
\hline 40 & .8 & .270 & .250 & 1.08 & .217 & .201 & 1.08 \\
\hline 60 & 0 & .167 & .167 & 1.00 & .003 & .003 & 1.00 \\
\hline 60 & .4 & .182 & .167 & 1.09 & .074 & .069 & 1.07 \\
\hline 60 & .8 & .182 & .167 & 1.09 & .136 & .134 & 1.01 \\
\hline
\end{tabular}

Note: Figures based on 5,000 simulations. See text for simulation details. 


\section{A.7 Details Comparing Design-Based and CRSE Estimators}

Using individual-level data, the CRSE variance estimator for the WLS coefficients for model (5) in the main text that includes baseline covariates is:

$$
\widehat{\operatorname{Var}}_{\mathrm{CRSE}}(\hat{\boldsymbol{\delta}})=g\left(\boldsymbol{Z}^{\prime} \boldsymbol{W} \boldsymbol{Z}\right)^{-1}\left(\sum_{b=1}^{h} \sum_{j=1}^{m_{b}} \boldsymbol{Z}_{j b}^{\prime} \boldsymbol{W}_{j b} \hat{\boldsymbol{e}}_{j b} \hat{\boldsymbol{e}}_{j b}^{\prime} \boldsymbol{W}_{j b} \boldsymbol{Z}_{j b}\right)\left(\boldsymbol{Z}^{\prime} \boldsymbol{W} \boldsymbol{Z}\right)^{-1}
$$

where $\boldsymbol{Z}$ is an $n \times(2 h+v)$ matrix with the full set of independent variables (the blockby-treatment status interactions, block indicators, and covariates); $\hat{\boldsymbol{\delta}}$ is the corresponding vector of coefficient estimates; $\boldsymbol{W}$ is an $n \times n$ symmetric weight matrix with diagonal entries $w_{i j b}$ and all other entries $0 ; \boldsymbol{W}_{j b}$ are $n_{j b} \times n_{j b}$ submatrices of $\boldsymbol{W}$ defined for each cluster; $\hat{\boldsymbol{e}}_{j b}$ is a vector of WLS residuals for each cluster; and $g$ is a small sample correction term discussed in Section 4.3 in the main text.

Consider a single block-by-treatment ATE estimator from the regression model. In this case, after some algebra, the CRSE variance estimator in (S8) reduces to

$$
\widehat{\operatorname{Var}}_{\mathrm{CRSE}}\left(\hat{\beta}_{1, b}\right)=g \frac{s_{D_{b}}^{2 *}(1)}{m_{b}^{1}}+g \frac{s_{D_{b}}^{2 *}(0)}{m_{b}^{0}}
$$

where $s_{D_{b}}^{2 *}(1)=\frac{m_{b}^{1}-1}{m_{b}^{1}} s_{D_{b}}^{2}(1)$ and $s_{D_{b}}^{2 *}(0)=\frac{m_{b}^{0}-1}{m_{b}^{0}} s_{D_{b}}^{2}(0)$. This establishes $(10)$ in the main text.

Next consider adding covariates to the model. In this case, using (S8), the CRSE variance estimator for $\hat{\beta}_{1, b}$ becomes

$$
\widehat{\operatorname{Var}}_{\mathrm{CRSE}}\left(\hat{\beta}_{1, b}\right)=g \frac{\sum_{s=1}^{h} \sum_{j=1}^{m_{s}} \hat{\boldsymbol{\xi}}_{j s, b}^{\prime} \boldsymbol{W}_{j s} \hat{\boldsymbol{e}}_{j s} \hat{\boldsymbol{e}}_{j b}^{\prime} \boldsymbol{W}_{j s} \hat{\boldsymbol{\xi}}_{j s, b}}{\left(\sum_{s=1}^{h} \sum_{j=1}^{m_{s}} \hat{\boldsymbol{\xi}}_{j s, b}^{\prime} \boldsymbol{W}_{j s} \hat{\boldsymbol{\xi}}_{j s, b}\right)^{2}}
$$

where $\hat{\boldsymbol{\xi}}_{j s, b}$ is the residual in block $s$ from a weighted regression of $S_{i j b} \tilde{T}_{j b}$ on the other block-by-treatment status interactions and covariates in $\boldsymbol{Z}$. In comparison, the design-based estimator in (9) of the main text can be expressed in a parallel matrix form as

$$
\begin{aligned}
\widehat{\operatorname{Var}}\left(\hat{D}_{b}\right)= & \frac{m_{b}^{1}}{\left(m_{b}^{1}-v^{*} p_{b}^{*} q_{b}^{*}-1\right)} \frac{\sum_{s=1}^{h} \sum_{j: T_{j s}=1}^{m_{s}} \hat{\boldsymbol{\eta}}_{j s, b}^{\prime} \boldsymbol{W}_{j s} \hat{\boldsymbol{e}}_{j s} \hat{\boldsymbol{e}}_{j b}^{\prime} \boldsymbol{W}_{j s} \hat{\boldsymbol{\eta}}_{j s, b}}{\left(\sum_{s=1}^{h} \sum_{j: T_{j s}=1}^{m_{s}} \hat{\boldsymbol{\eta}}_{j s, b}^{\prime} \boldsymbol{W}_{j s} \hat{\boldsymbol{\eta}}_{j s, b}\right)^{2}} \\
& +\frac{m_{b}^{0}}{\left(m_{b}^{0}-v^{*}\left(1-p_{b}^{*}\right) q_{b}^{*}-1\right)} \frac{\sum_{s=1}^{h} \sum_{j: T_{j s}=0}^{m_{s}} \hat{\boldsymbol{\eta}}_{j s, b}^{\prime} \boldsymbol{W}_{j s} \hat{\boldsymbol{e}}_{j s} \hat{\boldsymbol{e}}_{j b}^{\prime} \boldsymbol{W}_{j s} \hat{\boldsymbol{\eta}}_{j s, b}}{\left(\sum_{s=1}^{h} \sum_{j: T_{j s}=0}^{m_{s}} \hat{\boldsymbol{\eta}}_{j s, b}^{\prime} \boldsymbol{W}_{j s} \hat{\boldsymbol{\eta}}_{j s, b}\right)^{2}}
\end{aligned}
$$

where $\hat{\boldsymbol{\eta}}_{j s, b}$ is now the residual in block s from a weighted regression of $S_{i j b} \tilde{T}_{j b}$ on the other block-by-treatment status interactions, but not the covariates. As with the no covariate case, the two estimators differ in their degrees of freedom adjustments. However, they now also differ in the handling of the correlations between the treatment indicators and covariates $\left(\hat{\boldsymbol{\xi}}_{j s, b}\right.$ versus $\left.\hat{\boldsymbol{\eta}}_{j s, b}\right)$. The two variance estimators, however, are again asymptotically equivalent. 


\section{Appendix B: Detailed Simulation Methods and Results}

\section{Setup for primary simulations}

For the simulations, for each scenario considered, we created a single base dataset that included all potential outcomes and covariates, and then for each of 1,000 replications, we randomly assigned half the clusters to treatment and half to control, storing the associated outcomes. We then fit our models and recorded results. Finally, we calculated the following statistics: (1) Type 1 errors across the replications; (2) biases of the ATE estimators (which are the same for the two estimators); (3) average empirical values of the standard errors produced by the estimators relative to their "true" sampling variability as measured by standard deviations of the 1,000 ATE estimates; (4) mean squared errors (MSEs) of the estimated standard errors around the true values; and (5) power levels, assuming a true ATE of 0.5 standard deviations when generating the data. To avoid unusual base datasets, we repeated this process for 100 base datasets and calculated average statistics. For all WLS estimations, clusters were weighted by their sample sizes.

The data generating process in (17) of the main text was used for the primary simulations. We parameterized all error variances in terms of the intraclass correlation coefficient for the control group, $I C C_{0}=\sigma_{u}^{2} /\left(\sigma_{u}^{2}+\sigma_{e}^{2}\right)$, the explained variance of the covariates in the control group, $R_{0}^{2}$, and the variance of the outcome in the control group, $\sigma_{y_{0}}^{2}$. In addition, we assumed that the two model covariates were iid normal with the same variance, $\sigma_{x_{k}}^{2}=\sigma_{x}^{2}$ and the same parameter values, $\gamma_{k}=1$. Thus, because $R_{0}^{2}=2 \gamma_{k}^{2} \sigma_{x}^{2} / \sigma_{y_{0}}^{2}$, we have that $\sigma_{x}^{2}=R_{0}^{2} \sigma_{y_{0}}^{2} / 2$. Let $\sigma_{y_{0}^{*}}^{2}=\sigma_{y_{0}}^{2}-2 \sigma_{x}^{2}$ and assume that $\sigma_{\theta}^{2}=f \sigma_{u}^{2}$ for $f \geq 0$. We then have that $\sigma_{u}^{2}=\sigma_{y_{0}^{*}}^{2} I C C_{0}, \sigma_{e}^{2}=$ $\sigma_{y_{0}^{*}}^{2}\left(1-I C C_{0}\right)$, and $\sigma_{\theta}^{2}=f \sigma_{y_{0}^{*}}^{2} I C C_{0}$. Further, we set $\sigma_{y_{0}}^{2}=1$ so that the outcomes are in effect size units based on the standard deviation of the control group, which is common practice.

To apply the formulas and match real-world scenarios, we assumed that $I C C_{0}=.10, f=.10$, and $R_{0}^{2}=.30$. These assumptions yield simulation values of $\sigma_{x}^{2}=.15, \sigma_{u}^{2}=.07, \sigma_{\theta}^{2}=.007$, and $\sigma_{e}^{2}=.63$.

Separate simulations were conducted using $m=8$ to 50 clusters, split evenly between the treatment and control groups. We assumed $n_{j}$ individuals per cluster, where $n_{j}$ differed across clusters and was generated to be correlated with both $u_{j}$ and $\theta_{j}$. Specifically, we generated cluster sample sizes using $n_{j}=\mu+n_{j}^{*}+\delta_{1} u_{j}+\delta_{2} \theta_{j}$ (which we rounded), where $n_{j}^{*}$ is a random variable with mean 0 and variance $\sigma_{n^{*}}^{2}$. We induced correlations between $n_{j}$ and $u_{j}$ of $\rho_{n u}=.25$ and between $n_{j}$ and $\theta_{j}$ of $\rho_{n \theta}=.05$. We set $\mu=100$ (40 in our sensitivity analyses) and $\sigma_{n}=10$ (5 in our sensitivity analyses). In terms of these parameters, we have $\delta_{1}=\rho_{n u} \sigma_{n} / \sigma_{u}, \delta_{2}=$ $\rho_{n \theta} \sigma_{n} / \sigma_{\theta}$, and $\sigma_{n^{*}}^{2}=\sigma_{n}^{2}-\delta_{1}^{2} \sigma_{u}^{2}-\delta_{2}^{2} \sigma_{\theta}^{2}$.

We conducted the simulations separately assuming that all the random variables $\left(x_{i j 1}, x_{j 2}, u_{j}, \theta_{j}, e_{i j}, n_{j}^{*}\right)$ were drawn from three types of distributions: (1) normal, (2) chi-squared (to allow for some skewness), and (3) bi-modal. We used a chi-squared distribution with 3 degrees of freedom that we centered at 0 and normalized to match the variances for each random variable. To simulate a bi-modal distribution for each random variable $k$, we drew from a normal distribution, $N\left(-\sqrt{.5 \sigma_{k}^{2}}, .5 \sigma_{k}^{2}\right)$, with probability $1 / 2$ and from a normal distribution, $N\left(\sqrt{.5 \sigma_{k}^{2}}, .5 \sigma_{k}^{2}\right)$, with probability $1 / 2$. 


\section{Calculating statistical power}

We conducted additional simulations to explore the statistical power of the estimators. To do so, we used the same data generating process as in our main setup, except that we assumed an average treatment effect of 0.5 standard deviations. We selected an effect size of 0.5 , because it provided a range of power estimates across the number of clusters we considered $(m=8$ to 50 clusters). For each estimator, we calculated the power as the percentage of times a t-test rejected the null hypothesis at the 5\% level. As a benchmark, we included an estimate of power, which was based on a t-test that used the estimate of the true standard error from our simulation results. We conducted these simulations assuming a finite population framework and no covariates.

\section{Calculating efficiency when covariates have different between and within effects}

We adapted our main simulation setup to allow covariates to have different within and between effects. Rather than generating $x_{i j k}$ directly, we instead generated data separately for the between- and within-cluster components, $\bar{x}_{j k}$ and $\left(x_{i j k}-\bar{x}_{j k}\right)$. We then included them as separate covariates in (17) to generate the outcomes, allowing for different parameter values, $\gamma_{B k}$ and $\gamma_{W k}$. We assigned all covariates the same between and within component variances $\left(\sigma_{B k}^{2}=\sigma_{B}^{2}\right.$ and $\left.\sigma_{W k}^{2}=\sigma_{W}^{2}\right)$ and the same parameter value for the between and within effects in (17) $\left(\gamma_{B k}=\gamma_{B}\right.$ and $\left.\gamma_{W k}=\gamma_{W}\right)$.

As in the main setup, we parameterized the covariates using their explained variance in the control group, assuming $R_{0}^{2}=0.3$ for the group of covariates. Under these assumptions $R_{0}^{2}=$ $\frac{v\left(\gamma_{B}^{2} \sigma_{B}^{2}+\gamma_{W}^{2} \sigma_{W}^{2}\right)}{\sigma_{y_{0}}^{2}}$, where $v$, the number of covariates, was set to 1 or 5 . We further assumed an intraclass correlation coefficient for each covariate, $\rho_{X}$, equal to 0.5 , which implies that $\sigma_{B}^{2}=$ $\sigma_{W}^{2}=\frac{\sigma_{x}^{2}}{2}$. Under these additional assumptions, $\sigma_{B}^{2}=\sigma_{W}^{2}=\frac{R_{0}^{2} \sigma_{y_{0}}^{2}}{v\left(\gamma_{B}^{2}+\gamma_{W}^{2}\right)}$. We parameterized the relative strength of the within and between effects using the ratio $\frac{\gamma_{B}}{\gamma_{W}}$, which was set to $1.0,1.5$, or 2.0. We assumed normal distributions for all random variables.

Importantly, for estimation using the individual data, we included the $x_{i j k}$ covariates only, calculated by summing the between- and within-cluster covariate components. Stated differently, we used $\bar{x}_{j k}$ and $\left(x_{i j k}-\bar{x}_{j k}\right)$ to generate the data, but used $x_{i j k}$ with associated parameter, $\gamma_{k}$, for estimation. Using this approach, we calculated estimates of the true standard error for models using the individual and aggregate (cluster-level) data.

The full simulation results for the clustered, non-blocked RCT are presented in Appendix Tables B.1 to B.6 below. 
Table B.1. Finite population simulation results on Type 1 error, bias, and standard errors for models without covariates

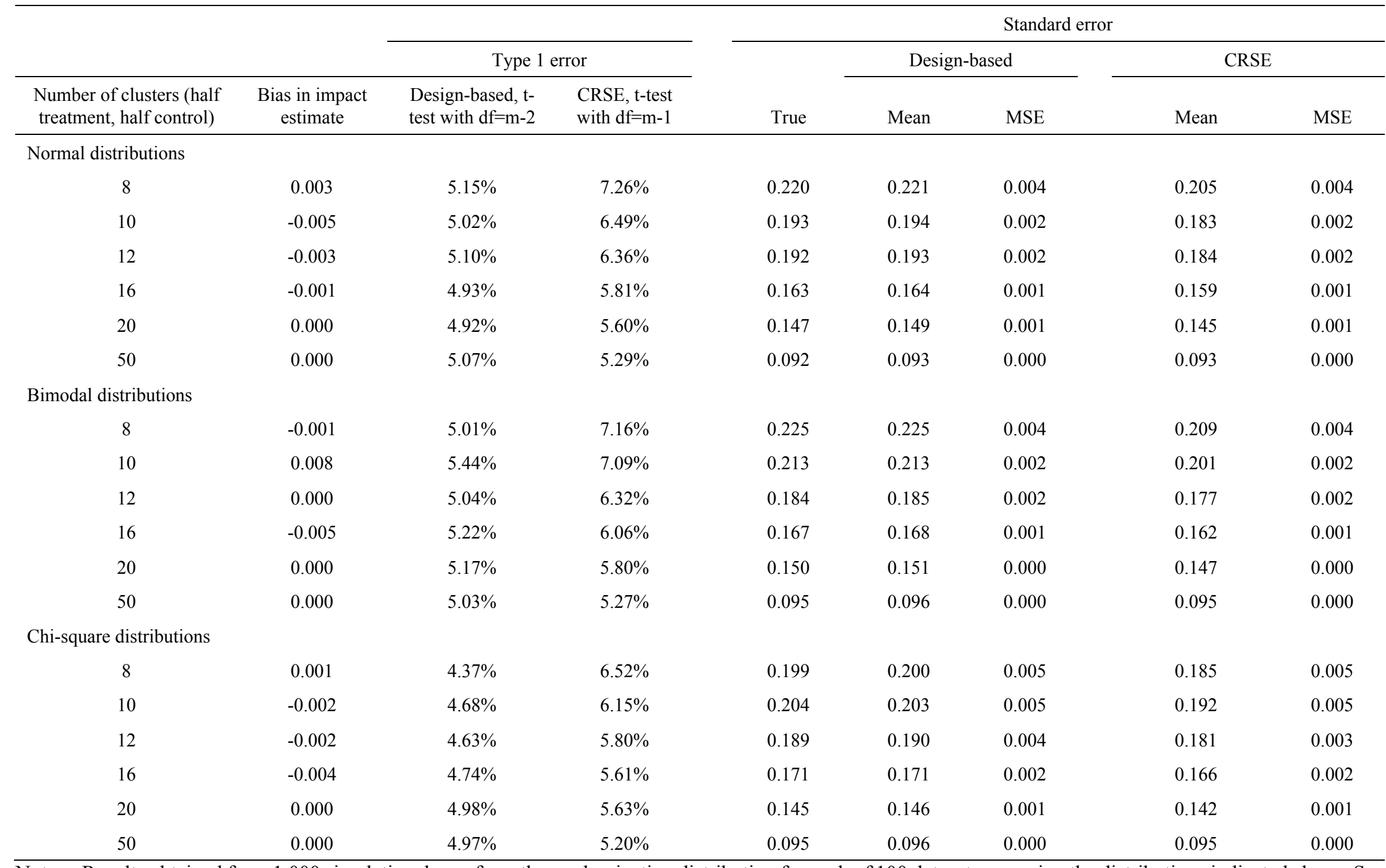

Notes: Results obtained from 1,000 simulation draws from the randomization distribution for each of 100 datasets assuming the distributions indicated above. See the main text and Appendix B for simulation specifications. The design-based variance estimator uses (9) in the main text. CRSE $=$ Standard cluster-robust standard error estimator; $\mathrm{df}=$ Degrees of freedom; MSE = Mean squared error. 
Table B.2. Finite population simulation results on power levels for models without covariates

Power

\begin{tabular}{|c|c|c|c|}
\hline $\begin{array}{l}\text { Number of clusters (half } \\
\text { treatment, half control) }\end{array}$ & $\begin{array}{c}\text { True, t-test with } \\
\qquad \mathrm{df}=\mathrm{m}-2\end{array}$ & $\begin{array}{l}\text { Design-based, t-test } \\
\text { with } \mathrm{df}=\mathrm{m}-2\end{array}$ & $\begin{array}{l}\text { CRSE, t-test } \\
\text { with } \mathrm{df}=\mathrm{m}-1\end{array}$ \\
\hline \multicolumn{4}{|l|}{ Normal distributions } \\
\hline 8 & $43.61 \%$ & $44.25 \%$ & $52.48 \%$ \\
\hline 10 & $59.47 \%$ & $57.85 \%$ & $63.77 \%$ \\
\hline 12 & $64.16 \%$ & $62.28 \%$ & $66.66 \%$ \\
\hline 16 & $81.09 \%$ & $77.28 \%$ & $79.61 \%$ \\
\hline 20 & $90.10 \%$ & $88.64 \%$ & $89.83 \%$ \\
\hline 50 & $99.98 \%$ & $99.97 \%$ & $99.97 \%$ \\
\hline \multicolumn{4}{|l|}{ Bimodal distributions } \\
\hline 8 & $42.00 \%$ & $40.13 \%$ & $48.00 \%$ \\
\hline 10 & $52.27 \%$ & $52.70 \%$ & $58.97 \%$ \\
\hline 12 & $68.16 \%$ & $62.67 \%$ & $67.10 \%$ \\
\hline 16 & $80.69 \%$ & $81.36 \%$ & $83.61 \%$ \\
\hline 20 & $89.35 \%$ & $89.33 \%$ & $90.51 \%$ \\
\hline 50 & $99.95 \%$ & $99.94 \%$ & $99.95 \%$ \\
\hline \multicolumn{4}{|l|}{ Chi-square distributions } \\
\hline 8 & $52.82 \%$ & $49.76 \%$ & $56.93 \%$ \\
\hline 10 & $55.28 \%$ & $56.85 \%$ & $61.81 \%$ \\
\hline 12 & $64.33 \%$ & $60.40 \%$ & $64.31 \%$ \\
\hline 16 & $79.64 \%$ & $79.78 \%$ & $81.68 \%$ \\
\hline 20 & $89.23 \%$ & $85.18 \%$ & $86.38 \%$ \\
\hline 50 & $99.91 \%$ & $99.75 \%$ & $99.76 \%$ \\
\hline
\end{tabular}

Notes: Results obtained from 1,000 simulation draws from the randomization distribution for each of 100 datasets assuming the distributions indicated above with a true average treatment effect of 0.5 standard deviations. See the main text and Appendix B for simulation specifications. The design-based variance estimator uses (9) in the main text. $\mathrm{CRSE}=$ Standard cluster-robust standard error estimator; $\mathrm{df}=$ Degrees of freedom. 
Table B.3. Finite population simulation results on Type 1 error, bias, and standard errors for models with covariates

\begin{tabular}{|c|c|c|c|c|c|c|c|c|c|c|c|}
\hline \multirow{3}{*}{$\begin{array}{l}\text { Number of clusters } \\
\text { (half treatment, } \\
\text { half control) }\end{array}$} & \multirow[b]{3}{*}{$\begin{array}{l}\text { Bias in impact } \\
\text { estimate }\end{array}$} & \multirow{2}{*}{\multicolumn{3}{|c|}{ Type 1 error }} & \multicolumn{7}{|c|}{ Standard error } \\
\hline & & & & & \multicolumn{3}{|c|}{ Design-based, $\mathrm{v}^{*}=0$} & \multicolumn{2}{|c|}{ Design-based, $v^{*}=2$} & \multicolumn{2}{|c|}{ CRSE } \\
\hline & & $\begin{array}{c}\text { Design- } \\
\text { based, } v^{*}=0\end{array}$ & $\begin{array}{c}\text { Design- } \\
\text { based, } v^{*}=2\end{array}$ & $\begin{array}{l}\text { CRSE, t-test } \\
\text { with } \mathrm{df}=\mathrm{m}-1\end{array}$ & True & Mean & MSE & Mean & MSE & Mean & MSE \\
\hline \multicolumn{12}{|c|}{ Two individual-level covariates with $\boldsymbol{R}_{\boldsymbol{T} \boldsymbol{X}}^{2}$ adjustment } \\
\hline \multicolumn{12}{|l|}{ Normal distributions } \\
\hline 8 & -0.002 & $4.98 \%$ & $1.31 \%$ & $7.40 \%$ & 0.187 & 0.188 & 0.003 & 0.230 & 0.006 & 0.174 & 0.003 \\
\hline 10 & -0.001 & $4.98 \%$ & $2.14 \%$ & $6.45 \%$ & 0.176 & 0.177 & 0.002 & 0.204 & 0.004 & 0.167 & 0.002 \\
\hline 12 & 0.002 & $5.12 \%$ & $2.84 \%$ & $6.41 \%$ & 0.154 & 0.155 & 0.001 & 0.174 & 0.002 & 0.148 & 0.001 \\
\hline 16 & 0.003 & $4.98 \%$ & $3.38 \%$ & $5.85 \%$ & 0.141 & 0.142 & 0.001 & 0.153 & 0.001 & 0.137 & 0.001 \\
\hline 20 & 0.001 & $5.10 \%$ & $3.79 \%$ & $5.76 \%$ & 0.124 & 0.125 & 0.000 & 0.133 & 0.001 & 0.122 & 0.000 \\
\hline 50 & -0.001 & $5.11 \%$ & $4.63 \%$ & $5.32 \%$ & 0.079 & 0.080 & 0.000 & 0.081 & 0.000 & 0.079 & 0.000 \\
\hline \multicolumn{12}{|c|}{ Chi-square distributions } \\
\hline 8 & -0.003 & $4.28 \%$ & $1.37 \%$ & $6.52 \%$ & 0.189 & 0.188 & 0.006 & 0.230 & 0.010 & 0.174 & 0.005 \\
\hline 10 & 0.004 & $4.49 \%$ & $1.90 \%$ & $5.93 \%$ & 0.172 & 0.172 & 0.004 & 0.199 & 0.006 & 0.162 & 0.004 \\
\hline 12 & -0.005 & $4.72 \%$ & $2.50 \%$ & $5.92 \%$ & 0.152 & 0.153 & 0.003 & 0.171 & 0.004 & 0.146 & 0.002 \\
\hline 16 & 0.000 & $4.84 \%$ & $3.15 \%$ & $5.65 \%$ & 0.140 & 0.140 & 0.001 & 0.152 & 0.002 & 0.136 & 0.001 \\
\hline 20 & 0.001 & $4.87 \%$ & $3.60 \%$ & $5.53 \%$ & 0.125 & 0.126 & 0.001 & 0.133 & 0.002 & 0.122 & 0.001 \\
\hline 50 & 0.001 & $5.06 \%$ & $4.54 \%$ & $5.31 \%$ & 0.081 & 0.082 & 0.000 & 0.084 & 0.000 & 0.081 & 0.000 \\
\hline \multicolumn{12}{|c|}{ Two cluster-level covariates with $\boldsymbol{R}_{\boldsymbol{T} \boldsymbol{X}}^{2}$ adjustment } \\
\hline \multicolumn{12}{|l|}{ Normal distributions } \\
\hline 8 & 0.000 & NA & $5.10 \%$ & $17.92 \%$ & 0.236 & NA & 0.010 & 0.229 & 0.011 & 0.162 & 0.011 \\
\hline 10 & 0.000 & NA & $4.80 \%$ & $12.94 \%$ & 0.197 & NA & 0.004 & 0.195 & 0.004 & 0.153 & 0.005 \\
\hline 12 & -0.002 & NA & $5.21 \%$ & $11.61 \%$ & 0.185 & NA & 0.002 & 0.184 & 0.002 & 0.152 & 0.003 \\
\hline 16 & 0.002 & NA & $5.16 \%$ & $8.92 \%$ & 0.151 & NA & 0.001 & 0.151 & 0.001 & 0.134 & 0.001 \\
\hline 20 & 0.003 & NA & $5.04 \%$ & $7.95 \%$ & 0.134 & $\mathrm{NA}$ & 0.001 & 0.134 & 0.001 & 0.122 & 0.001 \\
\hline
\end{tabular}




\begin{tabular}{|c|c|c|c|c|c|c|c|c|c|c|c|}
\hline \multirow{3}{*}{$\begin{array}{l}\text { Number of clusters } \\
\text { (half treatment, } \\
\text { half control) }\end{array}$} & \multirow[b]{3}{*}{$\begin{array}{c}\text { Bias in impact } \\
\text { estimate }\end{array}$} & \multirow{2}{*}{\multicolumn{3}{|c|}{ Type 1 error }} & \multicolumn{7}{|c|}{ Standard error } \\
\hline & & & & & \multicolumn{3}{|c|}{ Design-based, $\mathrm{v}^{*}=0$} & \multicolumn{2}{|c|}{ Design-based, $v^{*}=2$} & \multicolumn{2}{|c|}{ CRSE } \\
\hline & & $\begin{array}{c}\text { Design- } \\
\text { based, } \mathrm{v}^{*}=0\end{array}$ & $\begin{array}{c}\text { Design- } \\
\text { based, } \mathrm{v}^{*}=2\end{array}$ & $\begin{array}{l}\text { CRSE, t-test } \\
\text { with } \mathrm{df}=\mathrm{m}-1\end{array}$ & True & Mean & MSE & Mean & MSE & Mean & MSE \\
\hline 50 & 0.001 & NA & $5.20 \%$ & $6.00 \%$ & 0.081 & NA & 0.000 & 0.082 & 0.000 & 0.079 & 0.000 \\
\hline \multicolumn{12}{|c|}{ Chi-square distributions } \\
\hline 8 & 0.000 & NA & $5.26 \%$ & $18.04 \%$ & 0.232 & NA & 0.012 & 0.227 & 0.015 & 0.161 & 0.013 \\
\hline 10 & -0.005 & NA & $4.79 \%$ & $12.20 \%$ & 0.201 & NA & 0.006 & 0.200 & 0.007 & 0.158 & 0.007 \\
\hline 12 & -0.004 & NA & $4.81 \%$ & $10.39 \%$ & 0.172 & NA & 0.004 & 0.171 & 0.004 & 0.143 & 0.004 \\
\hline 16 & -0.001 & NA & $4.96 \%$ & $8.52 \%$ & 0.147 & NA & 0.002 & 0.147 & 0.002 & 0.130 & 0.002 \\
\hline 20 & -0.001 & NA & $5.02 \%$ & $7.64 \%$ & 0.131 & NA & 0.001 & 0.132 & 0.001 & 0.120 & 0.001 \\
\hline 50 & 0.000 & NA & $4.96 \%$ & $5.76 \%$ & 0.081 & NA & 0.000 & 0.082 & 0.000 & 0.079 & 0.000 \\
\hline
\end{tabular}

Notes: Results obtained from 1,000 simulation draws from the randomization distribution for each of 100 datasets assuming the distributions indicated above. See the main text and Appendix B for simulation specifications. The design-based variance estimator uses (9) in the main text. NA $=$ Not applicable, CRSE $=$ Standard cluster-robust standard error estimator; $\mathrm{df}=$ Degrees of freedom; $v^{*}=$ Degrees of freedom adjustment for the covariates for the DB estimator; MSE $=$ Mean squared error. 
Table B.4. Type 1 error rates for various model specifications

\begin{tabular}{|c|c|c|c|c|c|c|}
\hline \multirow[b]{2}{*}{ Model specification and estimator } & \multicolumn{6}{|c|}{ Number of clusters (half treatment, half control) } \\
\hline & 8 & 10 & 12 & 16 & 20 & 50 \\
\hline \multicolumn{7}{|c|}{ Design-based estimator (finite population (FP) heterogeneity term excluded except where otherwise noted) } \\
\hline $\begin{array}{l}\text { No covariates, average of } 40 \text { individuals } \\
\text { per cluster }\end{array}$ & $5.15 \%$ & $5.45 \%$ & $5.25 \%$ & $5.01 \%$ & $5.03 \%$ & $5.18 \%$ \\
\hline $\begin{array}{l}\text { Individual-level covariates, no } R_{T X}^{2} \\
\text { adjustment, } \mathrm{v}^{*}=0\end{array}$ & $4.99 \%$ & $5.01 \%$ & $5.12 \%$ & $4.99 \%$ & $5.11 \%$ & $5.11 \%$ \\
\hline FP heterogeneity term included & $6.01 \%$ & $5.63 \%$ & $5.67 \%$ & $5.45 \%$ & $5.44 \%$ & $5.21 \%$ \\
\hline $\begin{array}{l}\text { Individual-level covariates, no } R_{T X}^{2} \\
\text { adjustment, } \mathrm{v}^{*}=2\end{array}$ & $1.31 \%$ & $2.17 \%$ & $2.86 \%$ & $3.39 \%$ & $3.80 \%$ & $4.63 \%$ \\
\hline FP heterogeneity term included & $1.69 \%$ & $2.50 \%$ & $3.19 \%$ & $3.76 \%$ & $4.07 \%$ & $4.75 \%$ \\
\hline $\begin{array}{l}\text { Cluster-level covariates, no } R_{T X}^{2} \\
\text { adjustment, } \mathrm{v}^{*}=2\end{array}$ & $9.20 \%$ & $7.90 \%$ & $7.80 \%$ & $6.97 \%$ & $6.42 \%$ & $5.67 \%$ \\
\hline FP heterogeneity term included & $10.15 \%$ & $8.62 \%$ & $8.43 \%$ & $7.46 \%$ & $6.82 \%$ & $5.80 \%$ \\
\hline \multicolumn{7}{|l|}{$\begin{array}{l}1 \text { Individual-level, } 1 \text { cluster-level } \\
\text { covariate, } R_{T X}^{2} \text { adjustment }\end{array}$} \\
\hline $\mathrm{v}^{*}=0$ & $7.71 \%$ & $6.76 \%$ & $6.48 \%$ & $5.99 \%$ & $5.80 \%$ & $5.26 \%$ \\
\hline $\mathrm{v}^{*}=1$ & $5.16 \%$ & $5.04 \%$ & $5.11 \%$ & $5.06 \%$ & $5.04 \%$ & $5.03 \%$ \\
\hline $\mathrm{v}^{*}=2$ & $2.61 \%$ & $3.37 \%$ & $3.74 \%$ & $4.12 \%$ & $4.34 \%$ & $4.78 \%$ \\
\hline \multicolumn{7}{|l|}{ Standard CRSE estimator } \\
\hline $\begin{array}{l}\text { No covariates, average of } 40 \text { individuals } \\
\text { per cluster }\end{array}$ & $7.14 \%$ & $6.97 \%$ & $6.44 \%$ & $5.89 \%$ & $5.67 \%$ & $5.41 \%$ \\
\hline \multicolumn{7}{|l|}{$\begin{array}{l}\text { Individual-level covariates: alignment } \\
\text { with design-based estimator }\end{array}$} \\
\hline $\begin{array}{l}\text { Align degrees of freedom }(\mathrm{df}) \text { in } \\
\text { variance formulas, } \mathrm{v}^{*}=0\end{array}$ & $5.83 \%$ & $5.27 \%$ & $5.39 \%$ & $5.10 \%$ & $5.18 \%$ & $5.12 \%$ \\
\hline $\begin{array}{l}\text { Align df in variance formulas and t- } \\
\text { tests, } \mathrm{v}^{*}=0\end{array}$ & $5.06 \%$ & $4.99 \%$ & $5.12 \%$ & $4.99 \%$ & $5.11 \%$ & $5.11 \%$ \\
\hline \multicolumn{7}{|l|}{$\begin{array}{l}\text { Cluster-level covariates: alignment with } \\
\text { design-based estimator }\end{array}$} \\
\hline Align df in variance formulas, $\mathrm{v}^{*}=2$ & $10.21 \%$ & $7.60 \%$ & $7.49 \%$ & $6.22 \%$ & $5.84 \%$ & $5.29 \%$ \\
\hline $\begin{array}{l}\text { Align df in variance formulas and t- } \\
\text { tests, } \mathrm{v}^{*}=2\end{array}$ & $6.95 \%$ & $6.16 \%$ & $6.41 \%$ & $5.74 \%$ & $5.54 \%$ & $5.25 \%$ \\
\hline \multicolumn{7}{|l|}{$\begin{array}{l}1 \text { Individual-level and } 1 \text { cluster-level } \\
\text { covariate }\end{array}$} \\
\hline No alignment & $12.44 \%$ & $9.60 \%$ & $8.62 \%$ & $7.41 \%$ & $6.70 \%$ & $5.54 \%$ \\
\hline Align df in variance formulas, $\mathrm{v}^{*}=1$ & $8.19 \%$ & $6.72 \%$ & $6.28 \%$ & $5.73 \%$ & $5.46 \%$ & $5.10 \%$ \\
\hline $\begin{array}{l}\text { Align df in variance formulas and t- } \\
\text { tests, } v^{*=1}\end{array}$ & $6.56 \%$ & $5.89 \%$ & $5.70 \%$ & $5.45 \%$ & $5.28 \%$ & $5.07 \%$ \\
\hline
\end{tabular}

Notes: Results obtained from 1,000 simulation draws from the randomization distribution for each of 100 datasets assuming normal random variables. See the main text and Appendix B for simulation specifications. 
Table B.5. Finite population simulation results for true standard errors that allow for covariates to have different between and within effects

\begin{tabular}{|c|c|c|c|c|}
\hline \multirow[b]{2}{*}{$\begin{array}{c}\text { Number of clusters } \\
\text { (half treatment, half } \\
\text { control) }\end{array}$} & \multirow[b]{2}{*}{$\begin{array}{l}\text { Level of covariates included } \\
\text { in estimation }\end{array}$} & \multicolumn{3}{|c|}{$\begin{array}{l}\text { True standard error with varying within and between } \\
\text { effects: }\end{array}$} \\
\hline & & $\frac{\gamma_{B}}{\gamma_{W}}=1$ & $\frac{\gamma_{B}}{\gamma_{W}}=1.5$ & $\frac{\gamma_{B}}{\gamma_{W}}=2$ \\
\hline \multicolumn{5}{|c|}{1 covariate } \\
\hline \multirow[t]{2}{*}{8} & Individual & 0.197 & 0.203 & 0.225 \\
\hline & Cluster & 0.217 & 0.209 & 0.212 \\
\hline \multirow[t]{2}{*}{10} & Individual & 0.171 & 0.187 & 0.198 \\
\hline & Cluster & 0.182 & 0.189 & 0.187 \\
\hline \multirow[t]{2}{*}{12} & Individual & 0.160 & 0.169 & 0.175 \\
\hline & Cluster & 0.168 & 0.168 & 0.166 \\
\hline \multirow[t]{2}{*}{16} & Individual & 0.136 & 0.139 & 0.154 \\
\hline & Cluster & 0.141 & 0.137 & 0.142 \\
\hline \multirow[t]{2}{*}{20} & Individual & 0.120 & 0.129 & 0.136 \\
\hline & Cluster & 0.123 & 0.126 & 0.126 \\
\hline \multirow[t]{2}{*}{50} & Individual & 0.077 & 0.082 & 0.089 \\
\hline & Cluster & 0.078 & 0.080 & 0.082 \\
\hline \multicolumn{5}{|c|}{5 covariates } \\
\hline \multirow[t]{2}{*}{8} & Individual & 0.198 & 0.209 & 0.227 \\
\hline & Cluster & 0.795 & 0.800 & 0.805 \\
\hline \multirow[t]{2}{*}{10} & Individual & 0.179 & 0.190 & 0.207 \\
\hline & Cluster & 0.320 & 0.319 & 0.319 \\
\hline \multirow[t]{2}{*}{12} & Individual & 0.165 & 0.173 & 0.187 \\
\hline & Cluster & 0.235 & 0.236 & 0.237 \\
\hline \multirow[t]{2}{*}{16} & Individual & 0.143 & 0.151 & 0.163 \\
\hline & Cluster & 0.175 & 0.176 & 0.176 \\
\hline \multirow[t]{2}{*}{20} & Individual & 0.126 & 0.132 & 0.141 \\
\hline & Cluster & 0.145 & 0.145 & 0.145 \\
\hline \multirow[t]{2}{*}{50} & Individual & 0.080 & 0.084 & 0.089 \\
\hline & Cluster & 0.084 & 0.084 & 0.084 \\
\hline
\end{tabular}

Notes: Results obtained from 1,000 simulation draws from the randomization distribution for each of 100 datasets assuming the distributions indicated above. Simulations that include both the cluster-level covariates and within-cluster covariates produce the same results as those that include only the cluster-level covariates. 
Table B.6. Super-population simulation results on Type 1 error, bias, and standard errors for models without covariates

\begin{tabular}{|c|c|c|c|c|c|c|c|c|}
\hline \multirow[b]{3}{*}{$\begin{array}{l}\text { Number of clusters (half } \\
\text { treatment, half control) }\end{array}$} & \multirow[b]{3}{*}{$\begin{array}{l}\text { Bias in impact } \\
\text { estimate }\end{array}$} & \multicolumn{6}{|c|}{ Standard error } & \\
\hline & & \multicolumn{2}{|c|}{ Type 1 error } & & \multicolumn{2}{|c|}{ Design-based } & \multicolumn{2}{|c|}{ CRSE } \\
\hline & & $\begin{array}{l}\text { Design- based, t- } \\
\text { test with } \mathrm{df}=\mathrm{m}-2\end{array}$ & $\begin{array}{l}\text { CRSE, } t \text {-test } \\
\text { with } \mathrm{df}=\mathrm{m}-1\end{array}$ & & Mean & MSE & Mean & MSE \\
\hline \multicolumn{9}{|c|}{ Normal distributions } \\
\hline 8 & 0.000 & $4.99 \%$ & $7.10 \%$ & 0.240 & 0.229 & 0.005 & 0.212 & 0.005 \\
\hline 10 & 0.000 & $5.02 \%$ & $6.57 \%$ & 0.214 & 0.207 & 0.003 & 0.195 & 0.003 \\
\hline 12 & 0.000 & $5.14 \%$ & $6.40 \%$ & 0.196 & 0.190 & 0.002 & 0.181 & 0.002 \\
\hline 16 & 0.001 & $5.01 \%$ & $5.88 \%$ & 0.170 & 0.166 & 0.001 & 0.161 & 0.001 \\
\hline 20 & 0.000 & $5.01 \%$ & $5.70 \%$ & 0.152 & 0.149 & 0.001 & 0.145 & 0.001 \\
\hline 50 & 0.001 & $5.05 \%$ & $5.29 \%$ & 0.096 & 0.095 & 0.000 & 0.094 & 0.000 \\
\hline \multicolumn{9}{|c|}{ Bimodal distributions } \\
\hline 8 & 0.001 & $5.20 \%$ & $7.42 \%$ & 0.239 & 0.231 & 0.004 & 0.214 & 0.004 \\
\hline 10 & 0.000 & $5.26 \%$ & $6.83 \%$ & 0.215 & 0.208 & 0.002 & 0.196 & 0.003 \\
\hline 12 & 0.001 & $5.23 \%$ & $6.43 \%$ & 0.196 & 0.191 & 0.002 & 0.182 & 0.002 \\
\hline 16 & 0.001 & $5.06 \%$ & $5.92 \%$ & 0.170 & 0.167 & 0.001 & 0.161 & 0.001 \\
\hline 20 & 0.000 & $5.10 \%$ & $5.77 \%$ & 0.151 & 0.150 & 0.001 & 0.146 & 0.001 \\
\hline 50 & 0.000 & $5.24 \%$ & $5.47 \%$ & 0.096 & 0.095 & 0.000 & 0.094 & 0.000 \\
\hline \multicolumn{9}{|c|}{ Chi-square distributions } \\
\hline 8 & -0.001 & $4.44 \%$ & $6.52 \%$ & 0.245 & 0.224 & 0.009 & 0.207 & 0.008 \\
\hline 10 & 0.001 & $4.60 \%$ & $6.15 \%$ & 0.220 & 0.205 & 0.006 & 0.193 & 0.006 \\
\hline 12 & 0.000 & $4.41 \%$ & $5.65 \%$ & 0.200 & 0.189 & 0.004 & 0.181 & 0.004 \\
\hline 16 & 0.000 & $4.67 \%$ & $5.52 \%$ & 0.175 & 0.167 & 0.002 & 0.162 & 0.002 \\
\hline 20 & 0.000 & $4.65 \%$ & $5.34 \%$ & 0.156 & 0.151 & 0.002 & 0.147 & 0.002 \\
\hline 50 & 0.001 & $4.97 \%$ & $5.17 \%$ & 0.100 & 0.098 & 0.000 & 0.097 & 0.000 \\
\hline
\end{tabular}

Notes: Results obtained from 50,000 simulation draws assuming the distributions indicated above. See the main text and Appendix B for simulation specifications. The design-based variance estimator uses (9) in the main text. $\mathrm{CRSE}=$ Standard cluster-robust standard error estimator; $\mathrm{df}=\mathrm{Degrees}$ of freedom; $\mathrm{MSE}=$ Mean squared error. 


\section{References}

Greene, W. H. (2018). Econometric Analysis. Pearsonr, New York, NY, 8th edition.

Hartley, H. and Ross, A. (1954). Unbiased ratio estimators. Nature, 174(4423):270.

Li, X. and Ding, P. (2017). General forms of finite population central limit theorems with applications to causal inference. Journal of the American Statistical Association, 112(520):1759-1769.

Middleton, J. A. (2008). Bias of the regression estimator for experiments using clustered random assignment. Statistics $\&$ probability letters, 78(16):2654-2659.

Middleton, J. A. and Aronow, P. M. (2015). Unbiased estimation of the average treatment effect in cluster-randomized experiments. Statistics, Politics, and Policy, 6(1-2):39-75.

Pashley, N. E. (2019). Note on the delta method for finite population inference with applications to causal inference. arXiv preprint arXiv:1910.09062.

Scott, A. and Wu, C.-F. (1981). On the asymptotic distribution of ratio and regression estimators. Journal of the American Statistical Association, 76(373):98-102. 